\title{
Improving the efficiency of upconversion by light concentration using nanoparticle design
}

Topical Review

Madsen, S. P.; Christiansen, J.; Christiansen, Rasmus Ellebæk; Vester-Petersen, J.; Møller, S. H.; Lakhotiya, H.; Nazir, A.; Eriksen, E.; Roesgaard, S.; Sigmund, Ole

Total number of authors:

15

Published in:

Journal of Physics D: Applied Physics

Link to article, DOI:

10.1088/1361-6463/ab5553

Publication date:

2020

Document Version

Peer reviewed version

Link back to DTU Orbit

Citation (APA):

Madsen, S. P., Christiansen, J., Christiansen, R. E., Vester-Petersen, J., Møller, S. H., Lakhotiya, H., Nazir, A., Eriksen, E., Roesgaard, S., Sigmund, O., Lissau, J. S., Destouesse, E., Madsen, M., Julsgaard, B., \& Balling, P. (2020). Improving the efficiency of upconversion by light concentration using nanoparticle design: Topical Review. Journal of Physics D: Applied Physics, 53(7), [073001]. https://doi.org/10.1088/1361-6463/ab5553

\section{General rights}

Copyright and moral rights for the publications made accessible in the public portal are retained by the authors and/or other copyright owners and it is a condition of accessing publications that users recognise and abide by the legal requirements associated with these rights.

- Users may download and print one copy of any publication from the public portal for the purpose of private study or research.

- You may not further distribute the material or use it for any profit-making activity or commercial gain

- You may freely distribute the URL identifying the publication in the public portal 


\title{
Improving the efficiency of upconversion by light concentration using nanoparticle design
}

\author{
S P Madsen ${ }^{1}$, J Christiansen ${ }^{2}, \mathrm{R}$ E Christiansen ${ }^{3}$, J \\ Vester-Petersen ${ }^{1}$, S H Møller ${ }^{2}$, H Lakhotiya ${ }^{2}$, A Nazir ${ }^{2}$, E \\ Eriksen $^{2}$, S Roesgaard ${ }^{2}$, O Sigmund ${ }^{3}$, J S Lissau ${ }^{4}$, E

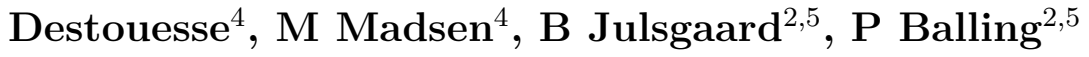 \\ ${ }^{1}$ Department of Engineering, Aarhus University, Inge Lehmanns Gade 10, DK-8000 \\ Aarhus C, Denmark. \\ ${ }^{2}$ Department of Physics and Astronomy, Aarhus University, Ny Munkegade 120, \\ DK-8000 Aarhus C, Denmark. \\ ${ }^{3}$ Department of Mechanical Engineering, Solid Mechanics, Technical University of \\ Denmark, Nils Koppels Allé, B. 404, DK-2800 Kgs. Lyngby, Denmark. \\ ${ }^{4}$ Mads Clausen Institute, University of Southern Denmark, Alsion 2, DK-6400 \\ Sønderborg, Denmark. \\ ${ }^{5}$ Interdisciplinary Nanoscience Center (iNANO), Aarhus University, Gustav Wieds \\ Vej 14, DK-8000 Aarhus C, Denmark. \\ E-mail: balling@phys.au.dk
}

4 September 2019

\begin{abstract}
Upconversion of sunlight with energy below the band gap of a solar cell is a promising technique for enhancing the cell efficiency, simply by utilizing a larger part of the solar spectrum. The present topical review addresses this concept and discusses the material properties needed for an efficient upconversion process with focus on both silicon and organic solar cells. To design efficient upconverters, insight into topics such as quantum-optics, nano-optics, numerical modeling, optimization, material fabrication, and material characterization is paramount, and the necessary concepts are introduced throughout the review. Upconversion modeling is done using rate equations, while optical modeling is done by solving Maxwell's equations using the finite element method. Topology optimization is introduced and used to generate geometries of gold nanoparticles capable of greatly enhancing the upconversion yield. Fabrication and experimental characterization methods are discussed. Some recent results are presented and finally the possibility of designing upconverting materials capable of increasing the short-circuit current in a solar cell is discussed.
\end{abstract}

Keywords: upconversion, topology optimization, field enhancement, photovoltaic Submitted to: J. Phys. D: Appl. Phys. 


\section{Introduction}

One of the fundamental limits on the efficiency of solar cells is their inability to absorb sunlight of wavelengths longer than that corresponding to the band gap of the absorber material. In crystalline silicon solar cells, this effect is responsible for losing about $20 \%$ of the energy from the sun. A potential solution to this problem is the process of upconversion, where two (or more) long-wavelength photons are converted to a photon with energy exceeding the band gap. This is inherently a non-linear process, which occurs with low probability under solar intensities. The density of absorbed energy can be adjusted in different ways. For an illustration, consider figure 1(a), which shows the "normal" case of radiation being absorbed in a material. If one employs a simple lens, as illustrated in figure 1(b), the incoming radiation can be concentrated into a smaller volume, which enhances the excitation density of each absorber and also allows for using a smaller amount of material. For many solar cell applications, e.g. rooftop panels, such an external concentration may be very inconvenient, and figure 1(c)

(a)
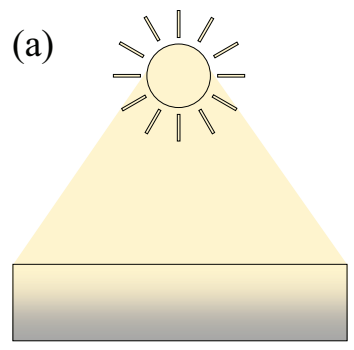

(d)

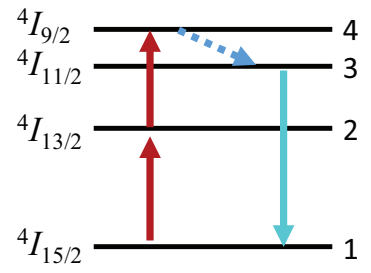

(b)
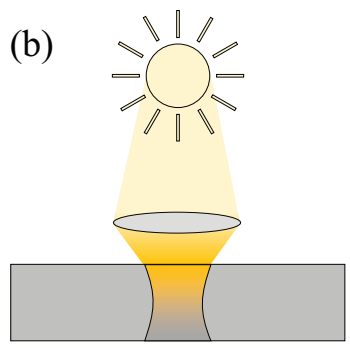

(e)

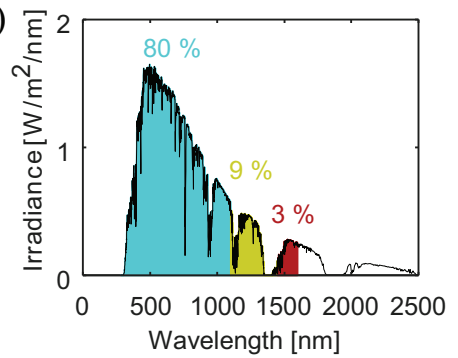

(c)
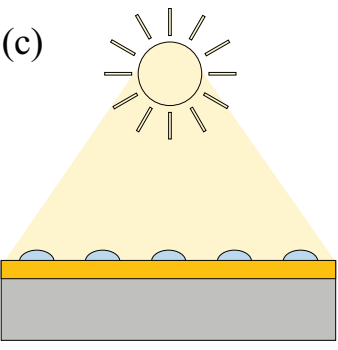

(f)

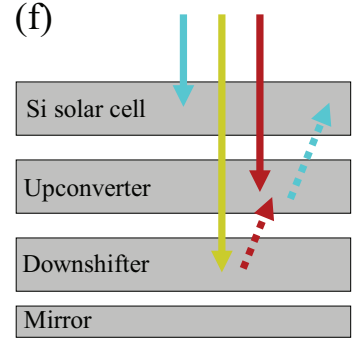

Figure 1. The upper three panels illustrate the absorption of (a) unconcentrated solar radiation, (b) externally concentrated solar radiation, and (c) radiation trapped and concentrated in a thin film by nanolenses. Panel (d) shows the four lowest energy levels in the $\mathrm{Er}^{3+}$ ion with two photons absorbed (upward arrows) at the wavelength $\sim 1.5 \mu \mathrm{m}$ leading to one upconverted photon (downward arrow) at a higher photon energy at the wavelength $\sim 980 \mathrm{~nm}$. The dashed arrow symbolizes non-radiative relaxation. Panel (e) shows the air-mass 1.5 solar spectrum, divided into parts that can be absorbed by a crystalline Si solar cell $(\lambda<1100 \mathrm{~nm}$, shown in blue), that can be envisaged to be absorbed in a downshifter material (1100 nm $<\lambda<1450 \mathrm{~nm}$, shown in yellow), and that can be absorbed in $\mathrm{Er}^{3+}$ ions $(1450 \mathrm{~nm}<\lambda<1600 \mathrm{~nm}$, shown in red). The relative energy content of each spectral range is also stated. Panel (f) illustrates the basic idea of an envisaged device, which efficiently converts the entire spectral range $1100 \mathrm{~nm}<\lambda<1600 \mathrm{~nm}$ to photons which can be directed back to a crystalline Si solar cell and absorbed. 
illustrates an alternative solution where a specially tailored set of nanolenses couple the incoming radiation into a thin film-again leading to concentration of the excitation density and usage of a smaller amount of absorbing material.

The topic of the present review is not the concentration of the entire solar spectrum, but instead the idea of using specially tailored nanolenses to concentrate an appropriately selected wavelength range around $1.5 \mu \mathrm{m}$ with the intent to better utilize the sub-bandgap photons, which are not absorbed in a crystalline silicon solar cell but instead are transmitted through it. This particular wavelength range corresponds to the absorbing range (typically 1450-1600 nm) of $\mathrm{Er}^{3+}$ ions, which have an energy-level structure as illustrated in figure 1(d). Upon absorption of two photons with wavelengths around $1.5 \mu \mathrm{m}$, a single photon with a shorter wavelength of $\sim 980 \mathrm{~nm}$ can be emitted. If this photon is directed back to the crystalline solar cell, it will generate one extra electron-hole pair and in turn increase short-circuit current and overall efficiency of the solar cell device. This is, however, only feasible provided that the upconversion process is efficient. In section 2 it will be explained how the concentration of incoming radiation relates to the efficiency of the upconversion process. Figure 1(e) shows the solar spectrum, which is divided into (colored) wavelength regions according to materials which can absorb the radiation. The blue part corresponds to the region that can be absorbed and hence utilized in a crystalline silicon solar cell. The narrow red band around $1.5 \mu \mathrm{m}$ is the absorption range of the $\mathrm{Er}^{3+}$ ion, which leaves a broad gap (yellow part) in the wavelength range $1100 \mathrm{~nm}<\lambda<1450 \mathrm{~nm}$. It has been proposed to fill this gap by using a downshifter material [1]. Downshifting is a process where one photon is absorbed followed by emission of another photon of lower energy, and the idea of [1] is to down-shift the yellow part of the spectrum in figure 1(e) to the region of $\mathrm{Er}^{3+}$ absorption. A rough sketch of a layered structure of different materials is presented in figure 1(f), illustrating how incoming radiation (downward arrows) is absorbed in different layers, spectrally converted to other wavelengths (upward dashed arrows), and finally reaching the Si solar cell absorber. For more details of the proposed geometry, we refer to [1], and the present review will not address this entire device structure. Instead the main focus is the very important sub-topic of reaching an efficient upconversion process. To do this, atomic-scale material models and nanoscale to microscale electromagnetic simulations are used. Section 2 discusses materials for upconversion based on $\mathrm{Er}^{3+}$ as well as molecular upconverters. In particular, a practical model of upconversion is presented. The model is based on rate equations and enables us to get a detailed insight regarding materials capable of efficient upconversion. Section 3 introduces concepts from optical theory and numerical modeling. These are needed for making advanced and accurate numerical models of the upconverting systems. Section 4 considers optimization based on the numerical models and introduces the concept of topology optimization for generating near-optimal nanoparticle shapes without an a priori assumed geometry. Section 5 considers the methods for fabrication of nanoparticles of possibly complicated shapes, their optical characterization as well as the verification of the numerical models by e.g. near-field mapping. Section 6 presents nanoparticle designs that result in high 
enhancement of the upconversion efficiency and presents the use of upconverters in organic solar cells, where nanoparticle enhancement has yet to be demonstrated. Section 7 concludes the review and discusses the possibilities of reaching the goal of designing and producing upconverting materials capable of delivering a significant increase in photo current in solar cells in conjunction with an efficient downshifter.

The main messages of the work presented in this review are: (i) It is possible to establish a theoretical model of the upconversion material and the concentration of incoming radiation by nano-lenses which, on the one hand, is simple enough to offer a clear and intuitive connection between material properties and energy efficiency of the upconversion process and, on the other hand, is accurate enough to reproduce the findings in experimental test devices. (ii) This theoretical model is computationally tractable to the extent that topological optimization of the nano-structures responsible for the lensing effect is possible. (iii) Real test devices have been realized and confirm our theoretical understanding. With these results at hand, we have presented a feasible scheme for future developments of optical structures for photon management facilitating the upconversion process, and we have reached a level of understanding on how to easily examine the material-limitations still to be overcome before a working device can be realized.

This review will not cover the entire device structure sketched in figure $1(\mathrm{f})$, which means specifically that the down-shifting material will not be described further. We refer to the $\mathrm{PhD}$ thesis of J. C. Goldschmidt [2] for further details of combining downshifting and upconverting materials. Although there is a good understanding in the literature of fluorescence concentrators [3], which are basically working down shifters, the main problem is that effecient materials are still to be developed for the spectral range $1100 \mathrm{~nm}<\lambda<1450 \mathrm{~nm}$. At present, the best candidate is PbS nanocrystals [4], which however are not environmentally friendly due to the presence of lead. In another approach, absorption of $1.1-1.45 \mu \mathrm{m}$ by $\mathrm{Ni}^{2+}$ ions and subsequent energy transfer to neighboring $\mathrm{Er}^{3+}$ ions has been realized to achieve broadband upconversion to $980 \mathrm{~nm}$ $[5,6]$. The present review will also not discuss spectral conversion of sun light in a broader sense, which can be found elsewhere [7]. The blue part of the solar spectrum in figure 1 represents $~ 80 \%$ of the incoming energy, but a very large portion of this is lost in crystalline silicon solar cells as heat since photons in the high-energetic part of the spectrum contributes a large kinetic energy to the generated electrons and holes. A better use of this part of the spectrum represents another line of research, using quantum cutting, where one high-energy photon is converted to two photons of lower energy, which in turn can generate two electron-hole pairs in the solar cell absorber [8]. Further information on a wide variety of upconversion materials for PV application can be found in $[9,10]$. For upconversion based on rare-earth ions, see e.g. $[11,12]$. 


\section{Materials for upconversion}

The ability to synthesize materials and understand their interaction with light is of utmost importance in the quest for a working upconversion device for improved solar cell performance. In the following, we describe in section 2.1 a simple analytical model for the interaction of the $\mathrm{Er}^{3+}$-based upconverter material with light, in section 2.2 the fabrication methods used in our research efforts, in section 2.3 an assessment of the feasibility of our specific $\mathrm{Er}^{3+}$-based upconverter materials for working devices, and in section 2.4 we discuss molecular upconverters. However, before entering into these details, it should be clarified which general properties we require from an efficient upconverter system. To this end, reconsider the solar spectrum in figure 1(e) and the sketched device in figure 1(f). The spectral region (1100 nm $<\lambda<$ $1450 \mathrm{~nm}$ ), marked with the yellow color in figure $1(\mathrm{e})$, represents a photon flux of $\Phi=\int_{1100 \mathrm{~nm}}^{1450 \mathrm{~nm}}(\lambda / h c) \operatorname{Ir}(\lambda) d \lambda \approx 5.4 \times 10^{20} \mathrm{~s}^{-1} \mathrm{~m}^{-2}$, where $h c / \lambda$ is the energy of one photon, $h$ is Planck's constant, $c$ is the speed of light, $\lambda$ is the wavelength, and $\operatorname{Ir}(\lambda)$ is the wavelength-dependent incoming irradiance shown in figure 1(e), corresponding to AM1.5 [13]. Assuming that an ideal downshifter transforms this photon flux with unit quantum efficiency to a wavelength of $1525 \mathrm{~nm}$, the resulting down-shifted intensity is $\approx 70 \mathrm{~W} / \mathrm{m}^{2}$, which is represented by the dashed red arrow in figure $1(\mathrm{f})$. Add to this the intensity $I=\int_{1450 \mathrm{~nm}}^{1600 \mathrm{~nm}} \operatorname{Ir}(\lambda) d \lambda \approx 31 \mathrm{~W} / \mathrm{m}^{2}$, which can be directly absorbed by the Er ions (red part of figure $1(\mathrm{e})$ and solid red arrow in figure $1(\mathrm{f}))$. As a resulting rule of thumb: A total incoming intensity (i.e. both red arrows in figure $1(\mathrm{f})$ ) of $I_{\mathrm{mnc}} \approx 100 \mathrm{~W} / \mathrm{m}^{2}$ is the absolute maximum non-concentrated (mnc) intensity that we can expect from an ideal down-shifter system and an ideal Si solar cell, which transmits all radiation not utilized by direct absorption. This $I_{\mathrm{mnc}}$ corresponds to $10 \%$ of the available solar irradiance.

One of the most studied upconverter materials is $\mathrm{NaYF}_{4}: \mathrm{Er}^{3+}$, i.e. a $\mathrm{NaYF}_{4}$ host crystal doped with Er such that typically $\approx 25 \%$ of the yttrium atoms in the host matrix are replaced by erbium [14]. This doping level corresponds to a density of $\mathrm{Er}^{3+}$ ions of $N \approx 3.5 \times 10^{21} \mathrm{~cm}^{-3}$. The absorption coefficient can then be calculated as $\alpha=\sigma_{12} N \approx 13 \mathrm{~cm}^{-1}$, where the cross section for absorption from the ground state to the first excited state (denoted simply as levels 1 and 2, see figure $1(\mathrm{~d})$ ) can be calculated as $[15]$

$$
\sigma_{12}=\frac{\lambda^{2} A_{21}}{8 \pi n_{\mathrm{R}}^{2}} S(\nu) \approx 3.8 \times 10^{-21} \mathrm{~cm}^{-2}
$$

Here $\lambda=1525 \mathrm{~nm}$ is the wavelength, $n_{\mathrm{R}}=1.48$ is the real part of the refractive index, $A_{21}=113.9 \mathrm{~s}^{-1}$ is the spontaneous emission rate (see appendix A in [16]), and $S(\nu)$ is the spectral function normalized such that $\int S(\nu) d \nu=1$ and $\nu$ is the frequency measured in Hertz. The absorption profile of $\mathrm{NaYF}_{4}: \mathrm{Er}^{3+}$ is approximately a Gaussian function centered at a wavelength of $1525 \mathrm{~nm}$ and with a FWHM of $90 \mathrm{~nm}$ (see figure $4.3 \mathrm{in} \mathrm{[16]),}$ leading to a value at resonance of $S(\nu) \approx 8 \times 10^{-14} \mathrm{~Hz}^{-1}$. Hence, the penetration depth is $\alpha^{-1} \approx 0.8 \mathrm{~mm}$ for resonant radiation at the center wavelength of $1525 \mathrm{~nm}$, which clearly demonstrates that the material is a very weak absorber. This represents one of the 


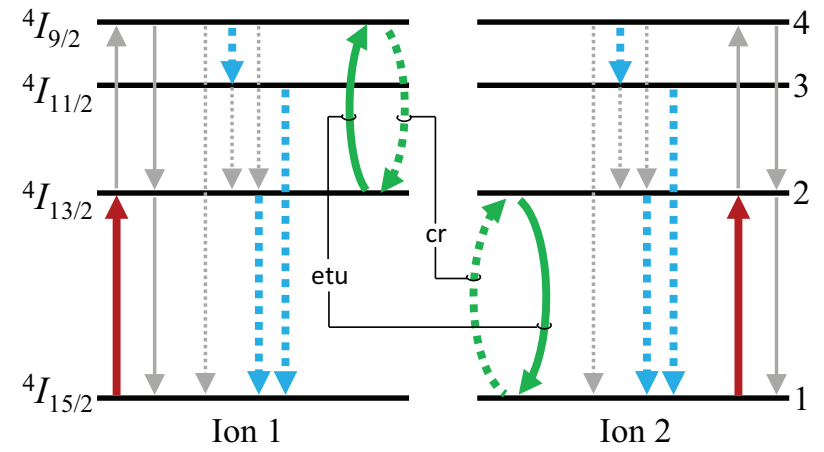

Figure 2. The four lowest energy levels of the $\mathrm{Er}^{3+}$-ion together with the processes connecting each level: Absorption at $1.5 \mu \mathrm{m}$ wavelength (upwards solid straight arrows), stimulated emission (downward solid straight arrows), radiative and non-radiative decay processes (downward dashed straight arrows), energy-transfer upconversion [etu] (solid curved arrows), and cross relaxation [cr] (dashed curved arrows). The upconverted photons of wavelength $980 \mathrm{~nm}$ are emitted on the ${ }^{4} I_{11 / 2} \rightarrow$ ${ }^{4} I_{15 / 2}$ transition. Processes, which are neglected in the simplified model described in section 2.1, are denoted by thin gray arrows, while the important processes are shown in color.

biggest challenges for achieving a practical upconverter device for solar cell applications based on $\mathrm{Er}^{3+}$.

\subsection{Rate-equation models}

In order to understand how much the incoming radiation must be concentrated to reach an efficient upconversion process, it is necessary to use a comprehensive model for the response of the upconverting material to radiation. Such a model was, for example, developed by S. Fischer, et al. [17], where a heroic attempt was made to either measure directly or fit indirectly every parameter in a rate-equation model, which then had to be solved numerically for modeling the upconversion process. More recently, we developed an analytical model, which involved a number of approximations and reached a set of simple expressions that describes the upconversion process well [18]. In the present subsection, the main equations of this analytical modeling will be stated together with a discussion of the results and their relation to an efficient upconversion device.

Denoting the four lowest energy levels of the $\operatorname{Er}^{3+}$ ion as 1, 2, 3, and 4, see figure $1(\mathrm{~d})$, and defining $\rho_{j}$ as the probability that an $\mathrm{Er}^{3+}$ ion populates the $j$ th level, a rate-equation model for the dynamics can be written as

$$
\begin{aligned}
& \frac{\partial \rho_{1}}{\partial t}=-\frac{\sigma_{12} I}{h \nu}+\Gamma_{21} \rho_{2}+\Gamma_{31} \rho_{3}+W_{\text {etu }} \rho_{2}^{2}-W_{\text {cr }} \rho_{4}, \\
& \frac{\partial \rho_{2}}{\partial t}=+\frac{\sigma_{12} I}{h \nu}-\Gamma_{21} \rho_{2}-2 W_{\text {etu }} \rho_{2}^{2}+2 W_{\text {cr }} \rho_{4}, \\
& \frac{\partial \rho_{3}}{\partial t}=-\Gamma_{31} \rho_{3}+\Gamma_{43} \rho_{4}, \\
& \frac{\partial \rho_{4}}{\partial t}=-\Gamma_{43} \rho_{4}+W_{\text {etu }} \rho_{2}^{2}-W_{\text {cr }} \rho_{4},
\end{aligned}
$$


where $\sigma_{12}$ is the absorption cross section mentioned in (1), $I$ is the intensity, $\nu$ is the frequency of the radiation, and $R_{12}=\sigma_{12} I / h \nu$ represents the single-ion absorption rate shown by the red arrow in figure 2. $\Gamma_{i j}$ is the total decay rate from level $i$ to level $j$, corresponding to the blue dashed arrows in figure 2 . The rate $W_{\text {etu }}$ corresponds to the energy transfer process shown with the solid green arrows in figure 2, where two adjacent $\mathrm{Er}^{3+}$ ions are initially found in level 2, but one ion takes up all the energy and transfers to level 4 while the other changes to level 1 . The rate of the opposite process, cross relaxation, is denoted by $W_{\text {cr }}$ and shown by the dashed green arrows in figure 2 . For the derivation and justification of the above equations, and their steady-state solution to be discussed below, we refer to [18]. It should be mentioned, however, that one key ingredient is the assumption that $\rho_{1} \approx 1$ and $\rho_{2,3,4} \ll 1$, i.e. the probability that any one $\mathrm{Er}^{3+}$ is excited is very small. This assumption requires the concentration of $\mathrm{Er}^{3+}$ to be sufficiently high such that the energy-transfer process dominates excited-state absorption and will be discussed further below.

The steady-state solution for the rate equations above is

$$
\begin{aligned}
& \rho_{2}=\frac{2 \sigma_{12} I_{\mathrm{sat}}}{h \nu \Gamma_{21}}\left(\sqrt{1+\frac{I}{I_{\mathrm{sat}}}}-1\right), \\
& \rho_{3}=\frac{\sigma_{12} I_{\mathrm{sat}}}{h \nu \Gamma_{31}}\left(1-\sqrt{1+\frac{I}{I_{\mathrm{sat}}}}+\frac{I}{2 I_{\mathrm{sat}}}\right), \\
& \rho_{4}=\frac{W_{\mathrm{etu}} \rho_{2}^{2}}{W_{\mathrm{cr}}+\Gamma_{43}},
\end{aligned}
$$

where the saturation intensity $I_{\text {sat }}$ is given by

$$
I_{\mathrm{sat}}=\frac{h \nu \Gamma_{21}^{2}\left(W_{\mathrm{cr}}+\Gamma_{43}\right)}{8 \sigma_{12} W_{\mathrm{etu}} \Gamma_{43}} .
$$

In turn, the rate of upconversion luminescence per ion is then

$$
\Gamma_{\mathrm{UCL}}=A_{31} \rho_{3}=\Gamma_{\mathrm{eff}}\left(1-\sqrt{1+\frac{I}{I_{\mathrm{sat}}}}+\frac{I}{2 I_{\mathrm{sat}}}\right),
$$

where $A_{31}$ is the radiative decay rate for the transition $3 \rightarrow 1$ and $\Gamma_{\text {eff }}=A_{31} \sigma_{12} I_{\text {sat }} / h \nu \Gamma_{31}$ is a convenient notation for brevity. Obviously, the saturation intensity $I_{\text {sat }}$ presents a mathematical demarcation point between different asymptotic regimes. For $I \ll I_{\text {sat }}$, the population $\rho_{3}$ and the upconversion luminescence rate grows quadratically with $I$, whereas for $I \gg I_{\text {sat }}$ the increase is only linear. In order to get a physical intuition of $I_{\text {sat }}$, it is illustrative to re-write the steady-state condition $\frac{\partial \rho_{2}}{\partial t}=0$ in the form

$$
\begin{aligned}
R_{12} & =\Gamma_{21} \rho_{2}+2 W_{\mathrm{etu}} \rho_{2}^{2}-2 W_{\mathrm{cr}} \rho_{4} \\
& =\Gamma_{21} \rho_{2}+2 \frac{W_{\mathrm{etu}} \Gamma_{43} \rho_{2}^{2}}{W_{\mathrm{cr}}+\Gamma_{43}} \\
& =\Gamma_{21} \rho_{2}+2 \Gamma_{31} \rho_{3},
\end{aligned}
$$

where all steps follow from using the steady-state condition with all derivatives in (2) being zero. The above equations describe an energy flow, or in other words, the destiny 
of the individual photons being absorbed at a rate of $R_{12}$ in each ion (on average). The physical interpretation of the term $\Gamma_{21} \rho_{2}$ is simply a loss channel, and the useful upconversion process must involve the non-linear term in the second line. One can interpret $W_{\text {etu }} \rho_{2}^{2}$ as the rate at which level 4 is populated and $\Gamma_{43} /\left(\Gamma_{43}+W_{\text {cr }}\right)$ as the branching ratio for subsequently reaching level 3 . Whenever an ion reaches level 3 via the energy-transfer mechanism, another ion must inevitably reach level 1, which is the reason for the factor of two in the non-linear term in the second line. For low excitation levels, the linear term $\Gamma_{21} \rho_{2}$ is dominant, and $\rho_{2} \propto R_{12}$, leading to a quadratic increase of $\rho_{3}$ and in turn a quadratic increase in the observed upconversion luminescence originating from level 3. In contrast, when the non-linear term becomes dominant, $\rho_{2} \propto \sqrt{R_{12}}$, and it becomes difficult to increase $\rho_{2}$ further-leading to only a further linear increase in the upconversion luminescence. It can easily be shown that the linear and quadratic terms in the above equations are equal when $I=8 I_{\text {sat }}$. Under these conditions, as illustrated by (13), $50 \%$ of the absorbed photons are directly wasted by ions, which decay directly to the ground level 1. Another $25 \%$ lead to a decay from level 3 to level 1 with the rate $\Gamma_{31} \rho_{3}$, while the last $25 \%$ correspond to decay from level 2 to level 1 as a part of the energy-transfer upconversion process. By defining the internal quantum efficiency (IQE) at the single-ion level as

$$
\mathrm{IQE}=\frac{\text { Rate of upconversion photon emission }}{\text { Rate of photon absorption }}=\frac{\Gamma_{\mathrm{UCL}}}{R_{12}},
$$

we thus conclude that when $I=8 I_{\text {sat }}$, then IQE $=25 \%$, provided that $\Gamma_{31}=A_{31}$ such that all decay events from level 3 are radiative. In other words, an appropriate target "working point" for a device for solar-cell improvement is the regime where the intensity $I$ is roughly an order of magnitude larger than $I_{\text {sat }}$. The concept of quantum efficiencies will be discussed further below.

Let us return to the discussion of the actual excitation level of each ion. Assume that, in an appropriately designed device, an intensity of $I=C \cdot I_{\mathrm{mnc}}$ is obtained, i.e. the maximum non-concentrated intensity $I_{\mathrm{mnc}}$ is concentrated by the factor $C$. Furthermore, take $A_{31}=149.7 \mathrm{~s}^{-1}$ from appendix A of [16] and calculate

$$
\frac{\rho_{3}}{C}=\frac{\sigma_{12} I_{\mathrm{mnc}} \lambda}{h c A_{31}} \cdot \mathrm{IQE} \approx 2 \times 10^{-6} \cdot \mathrm{IQE} .
$$

Since the IQE cannot exceed $50 \%$ (as it always takes two absorbed photons to create one upconverted photon), a value of $\approx 10^{-6}$ is an absolute upper bound for the ratio $\rho_{3} / C$ when using known parameters for $\mathrm{NaYF}_{4}: \mathrm{Er}^{3+}$. Since a concentration factor of $10^{6}$ seems very unrealistic, it must always hold for a working device based on this material that $\rho_{3} \ll 1$. In addition, since $A_{21}$ and $A_{31}$ are on the same order of magnitude, and since we must have $\Gamma_{21} \approx A_{21}$ and $\Gamma_{31} \approx A_{31}$ for an efficient device with negligible contribution from non-radiative decay channels, it can be concluded from (13) that $\rho_{2}$ and $\rho_{3}$ must be on the same order of magnitude under efficient working conditions (since the two terms of that equation are then equal in magnitude). In other words, $\rho_{2}$ and $\rho_{3}$ are both much smaller than unity under real device conditions and even smaller 

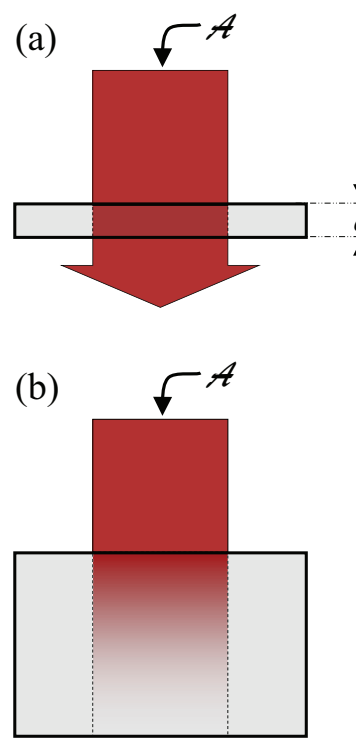

(c)

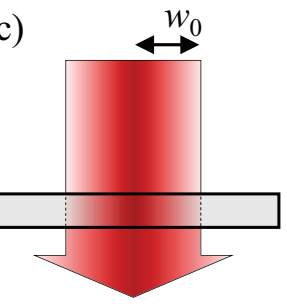

(d)

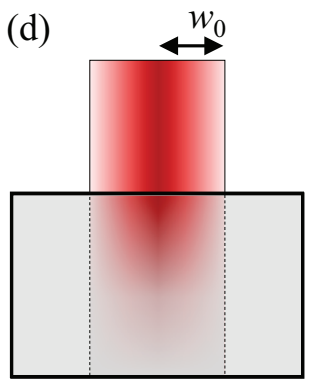

(e)

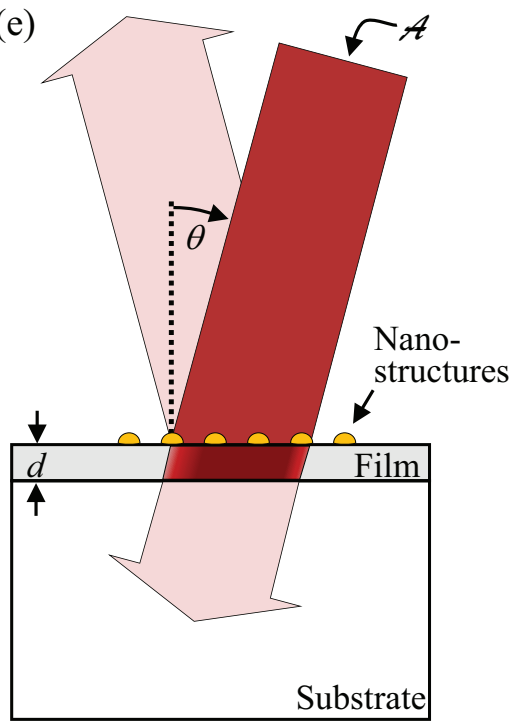

Figure 3. Various settings for calculating upconversion yield and absorption. Constant illumination on an area $\mathcal{A}$ of (a) a thin slice with thickness $d$ and (b) a thick slice of the upconverter material. Panels (c) and (d) illustrate a Gaussian beam of radius $w_{0}$ illuminating the same thin and thick slices as in panels (a) and (b), respectively. Panel (e) shows the main idea of using metal nanostructures to facilitate the coupling of an incoming beam of light into an upconverting film.

at weaker excitation densities. They may become large, however, if the material is subjected to intense laser radiation.

Now, in order to extend the above discussion of efficiency from the single-ion level to real materials, consider the different settings sketched in figure 3. This figure illustrates the incoming radiation (shown with red color) impinging on thin or thick slices of upconverting material or on a more complicated photonic structure. Consider first the simple case in figure 3(a), where an uniform illumination is incident on a slice of thickness $d$. If the total power of the incoming light is $P$, the characteristic intensity $\bar{I}$ in this problem is simply $\bar{I}=P / \mathcal{A}$, where $\mathcal{A}$ is the physical area of the illuminating beam. All ions experience the same intensity, and the upconversion yield, defined as the total number of upconverted photons emitted per second, can simply be calculated as

$$
Y_{\mathrm{UCL}}^{(\mathrm{a})}=N d \mathcal{A} \Gamma_{\mathrm{eff}} f^{(\mathrm{a})}\left(\bar{I} / I_{\mathrm{sat}}\right),
$$

where $f^{(\mathrm{a})}$ is given by

$$
f^{(\mathrm{a})}\left(\bar{I} / I_{\mathrm{sat}}\right)=1-\sqrt{1+\frac{\bar{I}}{I_{\mathrm{sat}}}}+\frac{\bar{I}}{2 I_{\mathrm{sat}}},
$$

and the super-script "(a)" refers to the corresponding panel of the setting in figure 3. Effects of reflection and thin-film interference have been ignored, and $\bar{I}$ is simply the characteristic intensity traveling through the thin slice. 
The total absorption rate, i.e. the number of absorbed photons per second in all ions, is given by

$$
Y_{\text {abs }}^{(\mathrm{a})}=R_{12} N d \mathcal{A}=\frac{\sigma_{12} \bar{I}}{h \nu} N d \mathcal{A},
$$

where $d \mathcal{A}$ is the volume of the illuminated slice and $N d \mathcal{A}$ thus corresponds to the total number of $\mathrm{Er}^{3+}$ ions being illuminated. The internal upconversion luminescence quantum yield can then be defined as the rate ratio of the upconverted and absorbed photons

$$
\operatorname{IUCQY}^{(\mathrm{a})}=\frac{Y_{\mathrm{UCL}}^{(\mathrm{a})}}{Y_{\mathrm{abs}}^{(\mathrm{a})}}=\frac{A_{31}}{\Gamma_{31}} \frac{I_{\mathrm{sat}}}{\bar{I}} f^{(\mathrm{a})}\left(\bar{I} / I_{\mathrm{sat}}\right),
$$

and the external quantum yield can be defined as the ratio of the upconversion luminescence yield and the incoming number of photons per second

$$
\operatorname{EUCQY}^{(\mathrm{a})}=\frac{Y_{\mathrm{UCL}}^{(\mathrm{a})}}{P / h \nu}=\operatorname{IUCQY}^{(\mathrm{a})} \cdot N d \sigma_{12},
$$

where the last factor $N d \sigma_{12}$ corresponds to the probability that a photon is absorbed while traversing through the slice of material.

Obviously, in an efficiently working device, both the internal and external upconversion quantum yields as well as the probability of photon absorption should approach unity. The simplest way to increase the probability of photon absorption is to merely increase the thickness of the absorber, corresponding to the sketched setup in figure 3(b), where the thickness of the material is assumed much larger than the penetration depth of the incoming radiation. In this case, the absorption probability is unity. The upconversion yield can be calculated as

$$
\begin{aligned}
Y_{\mathrm{UCL}}^{(\mathrm{b})} & =N \mathcal{A} \Gamma_{\text {eff }} \int_{0}^{\infty}\left(1-\sqrt{1+\frac{I(z)}{I_{\mathrm{sat}}}}+\frac{I(z)}{2 I_{\mathrm{sat}}}\right) d z \\
& =2 N \alpha^{-1} \mathcal{A} \Gamma_{\mathrm{eff}} f^{(\mathrm{b})}\left(\bar{I} / I_{\mathrm{sat}}\right)
\end{aligned}
$$

where $\alpha=N \sigma_{12}$ is the absorption coefficient and $I(z)=I_{0} e^{-\alpha z}$ is the intensity throughout the absorber material along the propagation direction, $z$. The $f^{(\mathrm{b})}$ function is given by

$$
f^{(\mathrm{b})}\left(\bar{I} / I_{\mathrm{sat}}\right)=1+\ln \left(\frac{\sqrt{1+2 \bar{I} / I_{\mathrm{sat}}}+1}{2}\right)-\sqrt{1+2 \bar{I} / I_{\mathrm{sat}}}+\frac{\bar{I}}{2 I_{\mathrm{sat}}},
$$

and the characteristic intensity is given by $\bar{I}=I_{0} / 2$, reflecting an averaging effect over the different intensities experienced by the different ions. With all light absorbed, $Y_{\mathrm{abs}}^{(\mathrm{b})}=P / h \nu=I_{0} \mathcal{A} / h \nu$, the internal quantum yield is equal to the external quantum yield and can be calculated to be

$$
\mathrm{IUCQY}^{(\mathrm{b})}=\mathrm{EUCQY}^{(\mathrm{b})}=\frac{A_{31}}{\Gamma_{31}} \frac{I_{\mathrm{sat}}}{\bar{I}} f^{(\mathrm{b})}\left(\bar{I} / I_{\mathrm{sat}}\right) .
$$



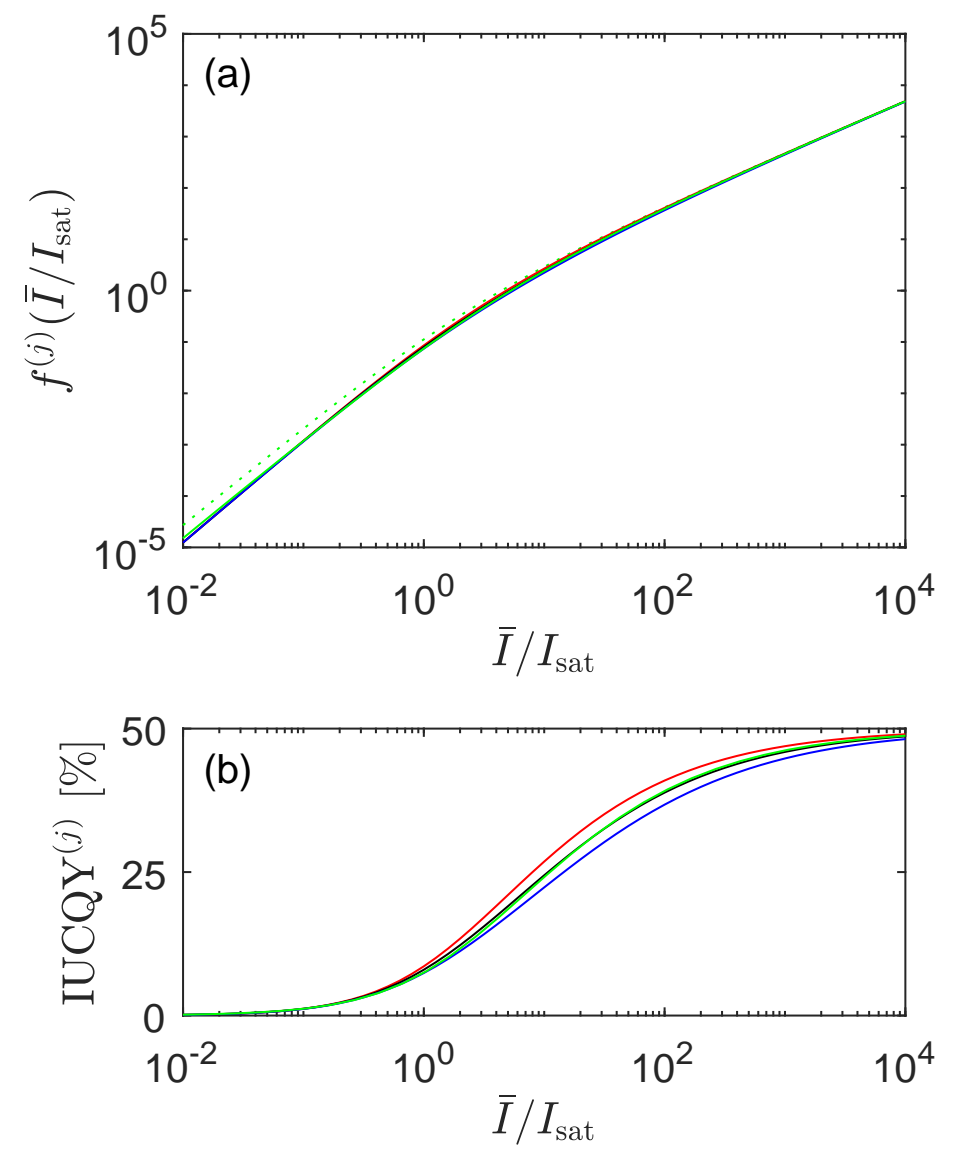

Figure 4. Panel (a) and (b) show, respectively, the $f^{(j)}$ and IUCQY ${ }^{(j)}$ functions versus $\bar{I} / I_{\text {sat }}$ for $j=(\mathrm{a})$ in red, $j=(\mathrm{b}, \mathrm{c})$ in black, $j=(\mathrm{d})$ in blue. In panel (a), the green dotted curve shows $\overline{f^{(a)}\left(|\boldsymbol{E}|^{2} / E_{\text {sat }}^{2}\right)}$ on the vertical axis and $|\boldsymbol{E}|^{2} / E_{\text {sat }}^{2}$ on the horizontal axis. The green solid curve shows a scaled version; $\frac{1}{\zeta} \overline{f^{(a)}\left(|\boldsymbol{E}|^{2} / E_{\mathrm{sat}}^{2}\right)}$ on the vertical axis and $|\boldsymbol{E}|^{2} / \zeta E_{\text {sat }}^{2}$ on the horizontal axis. In panel (b), the green curve shows the exact value of IUCQY ${ }^{(\mathrm{e})}$ from (33) on the vertical scale, but plotted as a function of $\overline{|\boldsymbol{E}|^{2}} / \zeta E_{\text {sat }}^{2}$ with $\zeta=0.53$.

Note that this expression is essentially the same as (19) with a slight modification in the definition of $\bar{I}$ and the $f$-function. For comparison, $f^{(\mathrm{a})}$ and $f^{(\mathrm{b})}$ are shown in figure $4(\mathrm{a})$, and the corresponding IUCQYs are shown in figure 4(b).

Another relevant case for fundamental studies on upconverter materials is shown in figure 3(c) and figure 3(d), where a spatially Gaussian laser beam is incident on, respectively, a thin and a thick sample. If the laser beam is collimated and not focused too tightly (i.e. with the Rayleigh range much longer than the absorber-material thickness), the spatial variations of the intensity can be described as

$$
I(r, z)=\frac{2 P}{\pi w_{0}^{2}} \exp \left(-\frac{2 r^{2}}{w_{0}^{2}}\right) e^{-\alpha z},
$$

where $w_{0}$ is the beam radius of the incident laser beam. The thin case in figure 3(c) corresponds to setting $z=0$ and was already derived in [18], leading to

$$
Y_{\mathrm{UCL}}^{(\mathrm{c})}=N d \pi w_{0}^{2} \Gamma_{\mathrm{eff}} f^{(\mathrm{c})}\left(\bar{I} / I_{\mathrm{sat}}\right),
$$


where the $f^{(\mathrm{c})}$ function is exactly the same as $f^{(\mathrm{b})}$, but with the characteristic intensity replaced by $\bar{I}=P / \pi w_{0}^{2}$. Furthermore, $Y_{\text {abs }}^{(\mathrm{c})}=N d \sigma_{12} P / h \nu$, leading to an expression for the IUCQY ${ }^{(\mathrm{c})}$ completely identical to $(24)$, as long as the above-mentioned $\bar{I}$ is used. The external quantum yield becomes EUCQY ${ }^{(\mathrm{c})}=\mathrm{IUCQY}^{(\mathrm{c})} \cdot N d \sigma_{12}$. These expressions for the IUCQY and EUCQY are important starting points for modeling experiments, where a Gaussian laser beam is incident on a thin film with nanostructures on top for increasing the light-trapping efficiency [19]. The case of the thick slice in figure 3 can conveniently be handled as a z-integral over (26), yielding

$$
\begin{aligned}
Y_{\mathrm{UCL}}^{(\mathrm{c})} & =N \pi w_{0}^{2} \Gamma_{\text {eff }} \int_{0}^{\infty} f^{(\mathrm{c})}\left(\frac{P}{\pi w_{0}^{2}} \frac{e^{-\alpha z}}{I_{\mathrm{sat}}}\right) d z \\
& =2 N \alpha^{-1} \pi w_{0}^{2} \Gamma_{\text {eff }} f^{(\mathrm{d})}\left(\bar{I} / I_{\text {sat }}\right),
\end{aligned}
$$

where the $f^{(\mathrm{d})}$ function must be calculated numerically and $\bar{I}=P / 2 \pi w_{0}^{2}$. Both IUCQY $^{(\mathrm{d})}$ and EUCQY ${ }^{(\mathrm{d})}$ are identical to (24), provided that the above value of $\bar{I}$ is used and $f^{(\mathrm{b})}$ is replaced by $f^{(\mathrm{d})}$. Laser experiments on thick samples (i.e. around $1 \mathrm{~mm}$ ) are not treated further in this review, but the above results were included for completeness. All above examples demonstrate, however, that the overall trend in the upconversion luminescence yield is similar for all configurations with only minor differences at the quantitative level. In the following, we seek to put the more complicated case from figure 3(e) into the same framework.

To this end, consider the setup in figure 3(e), where an upconverting film of thickness $d$ is placed on top of a substrate. An incoming beam of light is assumed for simplicity to hold a constant intensity profile across an area of $\mathcal{A}$, and the incidence angle is denoted by $\theta$. It can be convenient to express the intensities in terms of electric fields, which is the natural choice taken for the optical modeling in section 3 . For monochromatic fields, the relation between intensities and fields is $I=\frac{1}{2} \epsilon_{0} c n_{\mathrm{R}}|\boldsymbol{E}|^{2}$, where $\epsilon_{0}$ is the vacuum permittivity, $n_{\mathrm{R}}$ is the real part of the refractive index, and $\boldsymbol{E}$ is the complex electric field amplitude (with the physical field being the real part). The upconversion luminescence yield can then be calculated as

$$
Y_{\mathrm{UCL}}^{(\mathrm{e})}=N \Gamma_{\text {eff }} \int_{\text {film }} f^{(\mathrm{a})}\left(\frac{|\boldsymbol{E}(x, y, z)|^{2}}{E_{\mathrm{sat}}^{2}}\right) d V=\frac{N d \mathcal{A} \Gamma_{\mathrm{eff}}}{\cos \theta} \overline{f^{(\mathrm{a})}\left(\frac{|\boldsymbol{E}|^{2}}{E_{\text {sat }}^{2}}\right)} .
$$

The first expression is analogous to (16) but using integration over the non-constant electric field and changing the notation from intensities to fields. In the second step the bar over the $f$ function denotes an averaging over the film volume $V=d \mathcal{A} / \cos \theta$ and $(x, y, z)$ was left out for brevity. Likewise, the total absorption rate in the film must be given by

$$
Y_{\mathrm{abs}}^{(\mathrm{e})}=N \int_{\text {film }} R_{12} d V=\frac{\epsilon_{0} c n_{\mathrm{R}} \sigma_{12}}{2 h \nu} \frac{N d \mathcal{A}}{\cos \theta} \overline{|\boldsymbol{E}|^{2}},
$$

where the expression $R_{12}=\sigma_{12} I / h \nu$ was translated from intensity to electric field. Finally, the incoming number of photons per second is still $Y_{\text {in }}=P / h \nu$, and we proceed 
to calculate the absorption probability for an incoming photon as

$$
p_{\mathrm{abs}}=\frac{Y_{\mathrm{abs}}^{(\mathrm{e})}}{Y_{\mathrm{in}}}=N \sigma_{12} d \cdot \frac{\overline{|\boldsymbol{E}|^{2}} n_{\mathrm{R}}}{\left|E_{\mathrm{in}}\right|^{2} \cos \theta},
$$

where the incoming intensity was also translated to an incoming electric field amplitude $E_{\text {in }}$. This expression opens for a simple interpretation: The factor $N \sigma_{12} d$ is the usual expression for the probability of photon absorption, which is now modified by the concentration factor

$$
C_{\text {total }}=\frac{\overline{|\boldsymbol{E}|^{2}} n_{\mathrm{R}}}{\left|E_{\text {in }}\right|^{2} \cos \theta}=\frac{\overline{\left|\boldsymbol{E}_{\mathrm{b}}\right|^{2}} n_{\mathrm{R}}}{\left|E_{\mathrm{in}}\right|^{2} \cos \theta} \cdot \frac{\overline{|\boldsymbol{E}|^{2}}}{\overline{\left|\boldsymbol{E}_{\mathrm{b}}\right|^{2}}} \equiv C_{\text {film }} \cdot C_{\mathrm{ns}} .
$$

Here we used the following definition: Let $\boldsymbol{E}_{\mathrm{b}}(x, y, z)$ be the complex field amplitude within the film in the absence of nanostructures, denoted as the "background field". The factor $C_{\text {film }}=\overline{\left|\boldsymbol{E}_{\mathrm{b}}\right|^{2}} n_{\mathrm{R}} /\left|E_{\mathrm{in}}\right|^{2} \cos \theta$ is then a concentration factor, which solely deals with thin film interference and compares the intensity within the film (hence the $n_{\mathrm{R}}$ in the numerator) with the incoming intensity projected onto the film surface (hence the cosine factor in the denominator). In addition to this, the really interesting concentration factor $C_{\mathrm{ns}}=\overline{|\boldsymbol{E}|^{2}} / \overline{\left|\boldsymbol{E}_{\mathrm{b}}\right|^{2}}$ measures the separate impact of adding the nanostructures to the film and presents an essential parameter for the experiments in this work.

The procedure for calculating the electric fields within the upconverter material will be described in section 3, and the practical use of (33) for comparison with experiments will be discussed further in section 6 . For now we return to the desire of combining all examples of figure 3 into a common framework, and to this end, it is tempting to just apply the averaging within the $f^{(\mathrm{a})}$ function, i.e. to let $\overline{f^{(\mathrm{a})}(\beta)}=f^{(\mathrm{a})}(\bar{\beta})$. However, this is a poor approximation as illustrated by the green dotted curve in figure 4(a), which should coincide with the red curve if the approximation was good, but for small values of $\bar{I}$ the curve is more than a factor of two too large. For large fields or intensities, the approximation is exact due to the linearity of $f^{(a)}$. However, far below the saturation point the function is quadratic and the averaging inside the function thus underestimates the contribution from the high-intensity part of the distribution. Nonetheless, the green dashed curve in figure 4(a) has essentially the same shape as the other curves. Hence, in a practical experimental setting, one can easily obtain a satisfactory fit to measured upconversion luminescence yields from a nanostructure-covered film as shown in figure 3(e) but using the simple model function $Y_{\mathrm{UCL}}^{\mathrm{fit}}=A f^{(\mathrm{a})}\left(\bar{I} / I_{\mathrm{sat}}^{\text {on }}\right)$, where $\bar{I}=P / \mathcal{A}$ is the incoming intensity and $A$ and $I_{\text {sat }}^{\text {on }}$ are fitting parameters with "on" referring to the experimental situation where the illuminating light is "on" a region containing nanostructures. Thus, $I_{\text {sat }}^{\text {on }}$ refers to the experimental incoming intensity, which "drives the $\mathrm{Er}^{3+}$ ions into saturation" and thus represents a very practical way of characterizing the otherwise possibly complicated field $\boldsymbol{E}(x, y, z)$. For this reason, it is worthwhile to dig a little deeper into this. It was demonstrated in [19] that averaging $f$ could be done roughly as $\overline{f(\beta)} \approx \zeta f(\bar{\beta} / \zeta)$, where $\zeta$ is a number in the range $\left(\overline{|\boldsymbol{E}|^{2}}\right)^{2} / \overline{|\boldsymbol{E}|^{4}}<\zeta<1$. We refer to this reference for a detailed explanation and instead only show this scaling of $f^{(a)}$ as the green solid curve in figure 4(a). This curve essentially coincides with all the 
other curves to a reasonable precision, and the approximation enables us to write the IUCQY in a very convenient manner as

$$
\operatorname{IUCQY}^{(\mathrm{e})}=\frac{Y_{\mathrm{UCL}}^{(\mathrm{e})}}{Y_{\mathrm{abs}}^{(\mathrm{e})}}=\frac{A_{31}}{\Gamma_{31}} \frac{E_{\mathrm{sat}}^{2}}{\overline{|\boldsymbol{E}|^{2}}} \overline{f^{(\mathrm{a})}\left(\frac{|\boldsymbol{E}|^{2}}{E_{\mathrm{sat}}^{2}}\right)} \approx \frac{A_{31}}{\Gamma_{31}} \frac{I_{\mathrm{sat}}^{\mathrm{on}}}{\bar{I}} f^{(\mathrm{a})}\left(\frac{\bar{I}}{I_{\mathrm{sat}}^{\mathrm{on}}}\right),
$$

where $\bar{I} / I_{\text {sat }}^{\text {on }}=\overline{|\boldsymbol{E}|^{2}} / \zeta E_{\text {sat }}^{2}$. In figure $4(\mathrm{~b})$ the small difference between the red and green curves justify the above approximation, and we are left with an expression for the IUCQY that is identical to (19) and (24). Hence, the simple concept of a saturation intensity binds all the very different settings of figure 3 into a unified model. Moving the illuminating light "off" the nanostructure region and onto the bare film, would require the substitution of $\boldsymbol{E}$ by the much less varying background field $\boldsymbol{E}_{\mathrm{b}}$ and in turn replace $\overline{\left|\boldsymbol{E}_{\mathrm{b}}\right|^{2}} / E_{\text {sat }}^{2}$ by $\bar{I} / I_{\text {sat }}^{\text {off }}$ with a $\zeta$-value of unity. Hence, by not illuminating the nanostructures but only the bare film, the experimental fit to measured upconversion luminescence quantum yields, $Y_{\mathrm{UCL}}^{\mathrm{fit}}=A f^{(\mathrm{a})}\left(\bar{I} / I_{\text {sat }}^{\text {off }}\right)$, simply serves as a characterization of the upconverter material itself. This was essentially the topic of [18], which will be further discussed in section 2.3. At the same time, by fitting the upconversion luminescence yield obtained "on" the nanostructures and determining $I_{\text {sat }}^{\text {on }}$, it is possible to calculate

$$
\frac{I_{\text {sat }}^{\text {off }}}{I_{\text {sat }}^{\text {on }}}=\frac{\bar{I} / I_{\text {sat }}^{\text {on }}}{\bar{I} / I_{\text {sat }}^{\text {off }}}=\frac{\overline{|\boldsymbol{E}|^{2}} / \zeta E_{\text {sat }}^{2}}{\overline{\left|\boldsymbol{E}_{\mathrm{b}}\right|^{2}} / E_{\text {sat }}^{2}}=\frac{C_{\mathrm{ns}}}{\zeta},
$$

which can be thought of as an effective concentration factor of the nanostructures. The simple increase in electromagnetic energy density within the film is represented by $C_{\mathrm{ns}}$, and the factor $\zeta$ represents a small correction from the inhomogeneity of the energy density due to the non-linear response of the upconversion process. The actual enhancement of upconversion luminescence yield can be calculated by

$$
\text { Enhancement }=\frac{Y_{\mathrm{UCL}}^{(\mathrm{e}, \mathrm{on})}}{Y_{\mathrm{UCL}}^{(\mathrm{e}, \mathrm{off})}} \approx \zeta \frac{f^{(\mathrm{a})}\left(\bar{I} / I_{\mathrm{sat}}^{\mathrm{on}}\right)}{f^{(\mathrm{a})}\left(\bar{I} / I_{\mathrm{sat}}^{\text {off }}\right)},
$$

which depends strongly on $\bar{I}$, and this equation is thus important for understanding the relation between observed enhancement and light concentration. This topic was discussed thoroughly in [19], and will be re-visited in section 6 .

Finally, the above derivations enable the prediction of the external upconversion quantum yield. If the material parameter $I_{\text {sat }}^{\text {off }}$ is known, one finds

$$
\mathrm{EUCQY}^{(\mathrm{e})}=\operatorname{IUCQY}^{(\mathrm{e})} p_{\mathrm{abs}}=\frac{A_{31}}{\Gamma_{31}} \frac{I_{\mathrm{sat}}^{\mathrm{off}}}{\bar{I}} f^{(\mathrm{a})}\left(\frac{\bar{I}}{I_{\text {sat }}^{\text {off }}} \frac{C_{\mathrm{ns}}}{\zeta}\right) \cdot C_{\mathrm{film}} \zeta \cdot N \sigma_{12} d,
$$

where $C_{\text {ns }}$ and $C_{\text {film }}$ were defined around (32) and can be calculated by the methods in section 3, and $\zeta$ is a measure of the inhomogeneity of $\boldsymbol{E}$ within the film in the presence of nanostructures and typically equal to $\approx 0.5$. In the absence of nanostructures, simply set $C_{\mathrm{ns}}=\zeta=1$. As a final remark of this section, and as explained carefully in [19], the assumption of a constant illumination setting as shown in figure 3(e) can be replaced by a Gaussian beam illumination provided that we do the replacements $f^{(\text {a })} \rightarrow f^{(\mathrm{b})}$ and $\mathcal{A} \rightarrow \pi w_{0}^{2}$ in the above equations, i.e. $\bar{I} \rightarrow P / \pi w_{0}^{2}$. 


\subsection{Fabrication of upconverting materials}

Low phonon energy [20] and optical transparency in the visible and NIR spectral regions make fluorides, oxifluorides, and oxides suitable candidates for erbium-based upconversion processes. Ease of use, optical transparency, and high refractive index make $\mathrm{TiO}_{2}[21,22]$ our first host for the study. Furthermore, high upconversion efficiency and abundant research on the material $[16,23]$ make $\mathrm{NaYF}_{4}$ another suitable host for the erbium ions. In this work, $\mathrm{Er}^{3+}$-doped $\mathrm{TiO}_{2} / \mathrm{NaYF}_{4}$ systems are studied in their film configurations. We fabricated thin $(\approx 5 \mathrm{~nm})$ to reasonably thick $(\approx 300 \mathrm{~nm}) \mathrm{Er}^{3+}$-doped $\mathrm{TiO}_{2}$ films via radio-frequency magnetron sputtering. An AJA Orion ATC magnetron sputtering system with a sputtering power of $100 \mathrm{~W}$ was employed for the production of a large number of films. The sputtering target $\left(\mathrm{Er}_{2} \mathrm{O}_{3}+\mathrm{TiO}_{2}\right)$ was purchased with a controlled 5.1 at\% concentration of the Er dopant. The optical activity of the films was strongly dependent on the fabrication temperature inside the sputtering chamber [21]. The films deposited at $350{ }^{\circ} \mathrm{C}$ in $\mathrm{Ar}-\mathrm{O}_{2}(2$ at\%) atmosphere were found to be most luminescent $[21,24]$ and therefore employed for the present studies. The thickness of the films was controlled by the fabrication duration. A change in the thickness not only varied the total amount of $\mathrm{Er}^{3+}$ ions but also the optical properties of films due to thinfilm interference. Calculations have shown that the films with a thickness of $320 \mathrm{~nm}$ (adopted as thick $\mathrm{Er}^{3+}$-doped $\mathrm{TiO}_{2}$ films for the present studies) were most suitable for the plasmon-enhanced upconversion processes with a maximum in field strength near the film surface due to constructive interference between the incoming field and the field reflected from the film/substrate interface. The thickness and the compactness of $\mathrm{Er}^{3+}$-doped $\mathrm{TiO}_{2}$ films were characterized by transmission electron microscopy (TEM) via cross-sectional imaging using a FEI Talos F200X microscope. The cross sections of the films were prepared by focused-ion-beam milling.

Fabrication of $\mathrm{Er}^{3+}$-doped $\mathrm{NaYF}_{4}$ films is a two-step process as described in [25]. The first step involves chemical synthesis of $\mathrm{Er}^{3+}$-doped $\mathrm{NaYF}_{4} / \mathrm{NaLuF}_{4}$ core/shell nanocrystals (NCs) and the second step includes the film formation of such NCs by spin-coating on quartz substrates. It has been observed [26] that replacing yttrium by lutetium in the shell allows an easy and homogeneous tuning of the shell thickness around the core. The synthesis of core-shell NCs and the optimization of shells around the core are presented in [23]. We found that an increase in the shell thickness (up to $\approx 10 \mathrm{~nm}$ ) around the core (of diameter $\approx 20 \mathrm{~nm}$ ) increases the luminescence of the NCs [24]. The thinnest possible films (a monolayer) of each set of NCs were designed by drop-cast-assisted spin-coating. The experimental details are presented elsewhere $[25,23]$. The continuity and uniformity of the films, shown in figure $5(\mathrm{a}, \mathrm{b})$, were achieved by both controlling the NC concentration in the solution, optimizing the $\mathrm{NC}$ ligands and appropriate selection of solvent. The chemical synthesis is a multi-step process and each step consumes some amount of NCs that further reduces the overall production of the desired shelled NCs. For the thick film formation, we thus employed large-volume-produced-NCs with an intermediate shell thickness $(\approx 5 \mathrm{~nm})$, where the 

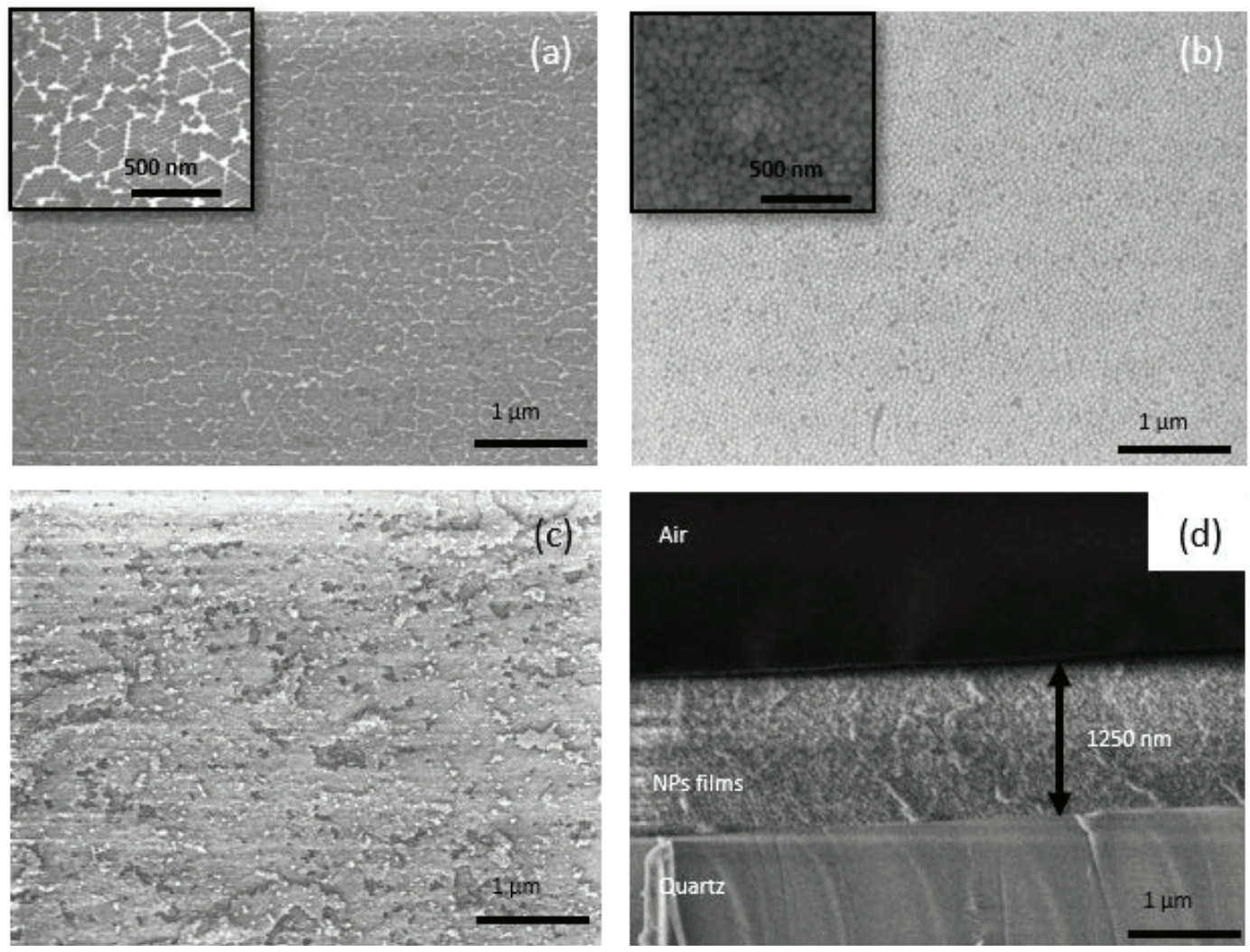

Figure 5. Scanning electron microscope imaging of thin $(a, b)$ and thick films $(c, d)$ of upconverting nanocrystals. Top-view imaging of monolayers of core (a) and core with shell thickness of $10 \mathrm{~nm}$ NCs (b). Top-view (c) and cross-sectional view (d) of 1250 $\mathrm{nm}$ thick films of NCs with shell thickness of $\approx 5 \mathrm{~nm}$. Insets of $(\mathrm{a}, \mathrm{b})$ are magnified images.

synthesis was performed in only two-steps. The structural and optical characterizations of such nanocrystals were performed by high-resolution TEM, energy dispersive X-ray spectroscopy (EDX), X-ray diffraction (XRD), as well as upconversion- and photoluminescence. Fabrication of the micron-size-thick $(1.25 \mu \mathrm{m})$ films was dependent on both the concentration of NCs in solution and solvent. The concentration was optimized and toluene as a solvent was chosen for the present studies. It is worth mentioning that the micron-sized thick films were fabricated without any external host materials. The ionic interaction between the NCs through the ligands could be a possible reason of the film formation. The compactness of the film was measured by top-view scanning electron microscope (SEM) shown in figure $5(\mathrm{c})$. The surface roughness was $\approx 5 \%$ of the film thickness, measured by cross-sectional SEM presented in figure 5(d).

\section{3. $E r^{3+}$-based upconverters}

The following subsection is dedicated to the upconversion properties of the two erbiumbased upconversion materials presented, that is, core-shell nanoparticles of $\mathrm{NaYF}_{4}: \operatorname{Er}$ and rf-magnetron sputtered thin films of $\mathrm{TiO}_{2}$ :Er. Due to the low absorption of trivalent 
erbium and the limited thickness of the studied thin films, we have not been able to quantify the erbium absorption and hence cannot determine the IUCQY directly via the ratio $Y_{\mathrm{UCL}} / Y_{\mathrm{abs}}$. However, we are able to measure the intensity dependence, the lifetimes of the two lowest excited states, ${ }^{4} I_{11 / 2}$ and ${ }^{4} I_{13 / 2}$, as well as the external quantum efficiency, which in combination with reported radiative decay constants in [16] allow us to asses the validity of the model presented in section 2.1 by testing the prediction in (36).

Time-resolved photoluminescence measurements have been conducted to determine the excited-state decay constants for the two materials. These measurements are carried out by exciting the samples with pulsed $800 \mathrm{~nm}$ laser radiation and subsequently measuring the luminescence centered at $977 \mathrm{~nm}$ or $1535 \mathrm{~nm}$ as a function of the delay from the pump pulse. The obtained decay curves are shown in figure 6 , and decay constants have been determined by fitting the obtained decay curves with appropriate models. For both materials the decay curves from the ${ }^{4} I_{13 / 2} \rightarrow{ }^{4} I_{15 / 2}$ transition are fitted nicely with a "single-exponential-with-rise model", $f(t)=[1-$ $\left.A_{\mathrm{R}} \exp \left(-g_{\mathrm{R}} t\right)\right] A_{1} \exp \left(-g_{1} t\right)$, interpreting $\Gamma_{21}=g_{1}$. The rise factor in the square brackets is present since relaxation from the pumped ${ }^{4} I_{9 / 2}$ level via the ${ }^{4} I_{11 / 2}$ level must occur before any significant luminescence from the ${ }^{4} I_{13 / 2} \rightarrow{ }^{4} I_{15 / 2}$ transition can be detected. The observed ${ }^{4} I_{11 / 2} \rightarrow{ }^{4} I_{15 / 2}$ decay of $\mathrm{TiO}_{2}$ : Er is fitted well by a bi-exponential model, $f(t)=A_{1} \exp \left(-g_{1} t\right)+A_{2} \exp \left(-g_{2} t\right)$, and the ${ }^{4} I_{11 / 2} \rightarrow{ }^{4} I_{15 / 2}$ decay of $\mathrm{NaYF}_{4}$ :Er is reasonably fitted by a "bi-exponential-with-rise model", $f(t)=$ $\left[1-A_{\mathrm{R}} \exp \left(-g_{\mathrm{R}} t\right)\right]\left(A_{1} \exp \left(-g_{1} t\right)+A_{2} \exp \left(-g_{2} t\right)\right)$. For these bi-exponential cases, the $\Gamma_{31}$ decay constant is chosen as the $g_{1}$ or $g_{2}$ value which belongs to the dominant decay term. Since it is the trailing part of the decay curves, which determines the decay constants, the small deviations between observations and curve fits in the initial part of the decay curves are not important. All the determined decay constants are stated in table 1 .

By comparing the values obtained with the radiative decay constants stated in [16], we see that the $\mathrm{NaYF}_{4}$ :Er is almost radiatively limited for the ${ }^{4} I_{13 / 2} \rightarrow{ }^{4} I_{15 / 2}$ transitions, meaning that no significant non-radiative decay occurs. This is unfortunately not the case for the ${ }^{4} I_{11 / 2} \rightarrow{ }^{4} I_{15 / 2}$ transition, where we can conclude from the quantum efficiency $\left(A_{31} / \Gamma_{31}\right)$ that only around $10 \%$ of the erbium ions relax radiatively; there is thus room for improvements. Assuming (for the lack of better) that the radiative decay constants, $A_{21}$ and $A_{31}$, taken from [16] are valid also for $\mathrm{TiO}_{2}$ : Er, the results in table 1 indicate the presence of significant non-radiative decay processes in $\mathrm{TiO}_{2}$ : Er, consistent with the findings in [21].

Upconversion spectra have been measured by exciting the sample with a $1500 \mathrm{~nm}$ cw-laser while varying the excitation intensity with attenuation filters. The UCL yield is computed by integrating the obtained UCL intensity emanating from the ${ }^{4} I_{11 / 2} \rightarrow{ }^{4} I_{15 / 2}$ transition, that is, computing the area of the UCL peak centered around $980 \mathrm{~nm}$. The saturation intensity is obtained by fitting the UCL yield against excitation intensity with the model shape of (23). The obtained values for the saturation intensity are stated 

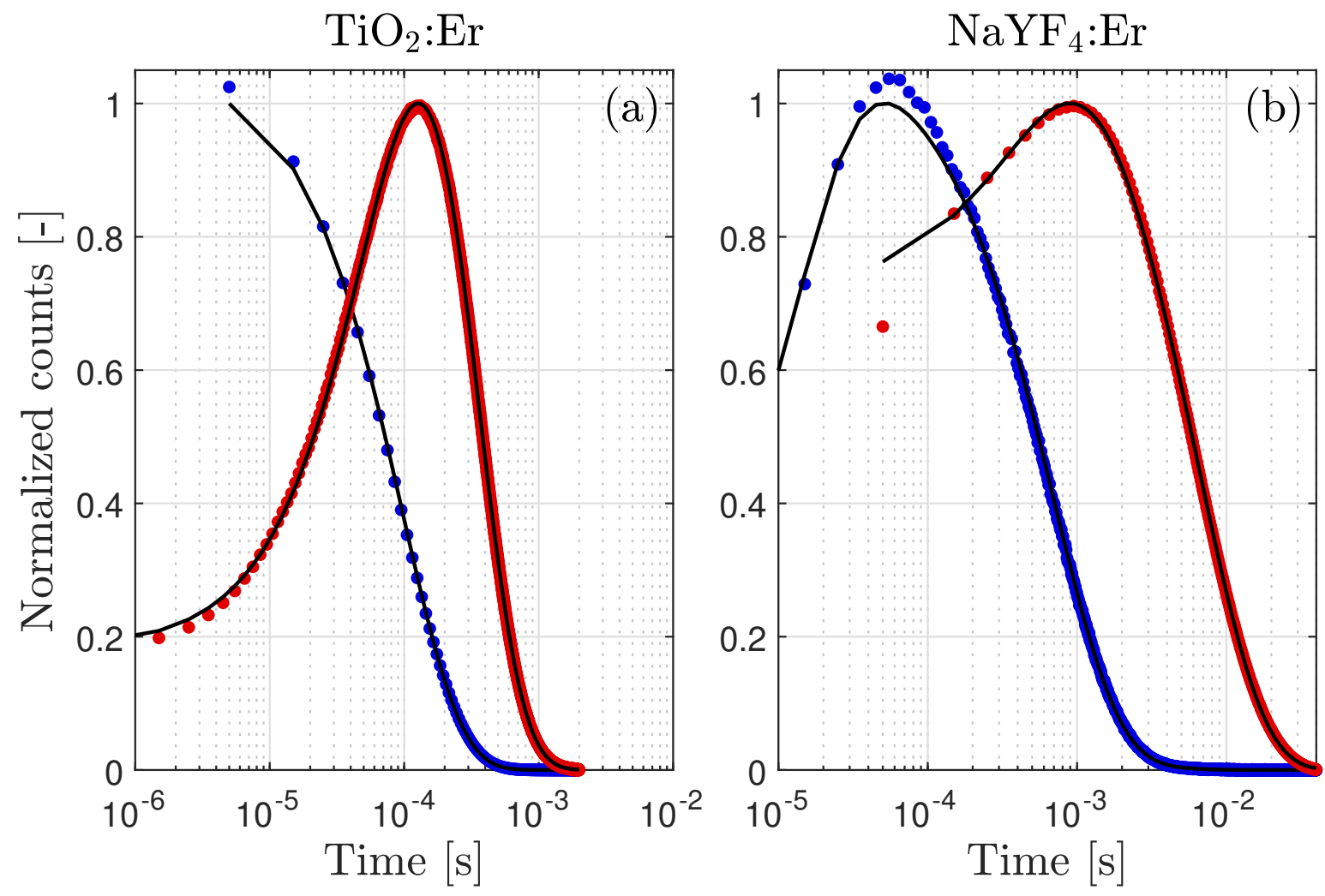

Figure 6. Photoluminescence decay curves for the ${ }^{4} I_{11 / 2} \rightarrow{ }^{4} I_{15 / 2}$ transition (blue) and the ${ }^{4} I_{13 / 2} \rightarrow{ }^{4} I_{15 / 2}$ transition (red) for both upconverter materials. The decay constants have been determined by fitting the decay curves with the appropriate models (black) as explained in the main text.

\begin{tabular}{lllll} 
& $\Gamma_{31}\left[\mathrm{~s}^{-1}\right]$ & $\Gamma_{21}\left[\mathrm{~s}^{-1}\right]$ & $I_{\text {sat }}\left[\mathrm{W} / \mathrm{cm}^{2}\right]$ & EUCQY $[-]$ \\
\hline $\mathrm{TiO}_{2}: \operatorname{Er}$ & $1.04(1) \cdot 10^{4}$ & $4.30(4) \cdot 10^{3}$ & $40(11)$ & $1.50(7) \cdot 10^{-6}\left(@ 4.06 \cdot 10^{2} \mathrm{~W} / \mathrm{cm}^{2}\right)$ \\
$\mathrm{NaYF}_{4}: \operatorname{Er}$ & $1.46(2) \cdot 10^{3}$ & $1.54(2) \cdot 10^{2}$ & $0.46(14)$ & $7.3(4) \cdot 10^{-6}\left(@ 2.16 \cdot 10^{1} \mathrm{~W} / \mathrm{cm}^{2}\right)$ \\
\hline
\end{tabular}

Table 1. Experimental parameters for the two upconverter materials based on optical investigations.

in table 1. If we compare the two upconverter materials by their saturation intensity, it is thus evident that $\mathrm{NaYF}_{4}$ :Er is a better upconverter since, as discussed in section 2.1, a good upconverter has a low saturation intensity. The significant contribution of non-radiative decay processes in $\mathrm{TiO}_{2}$ : Er is believed to be the main reason for the different saturation intensities [18].

Finally, the external quantum efficiencies of the two materials have been measured at well-chosen excitation intensities, yielding good signal-to-noise ratios. The measurements are carefully calibrated using an integrating sphere and are stated in table 1. Since this EUCQY $=Y_{\mathrm{UCL}} /(P / h \nu)$, and since we know the scaling of $Y_{\mathrm{UCL}}$ and $P$ from the experimentally determined upconversion spectra and excitation power, respectively, it is possible to re-calibrate the measured upconversion yield in terms 

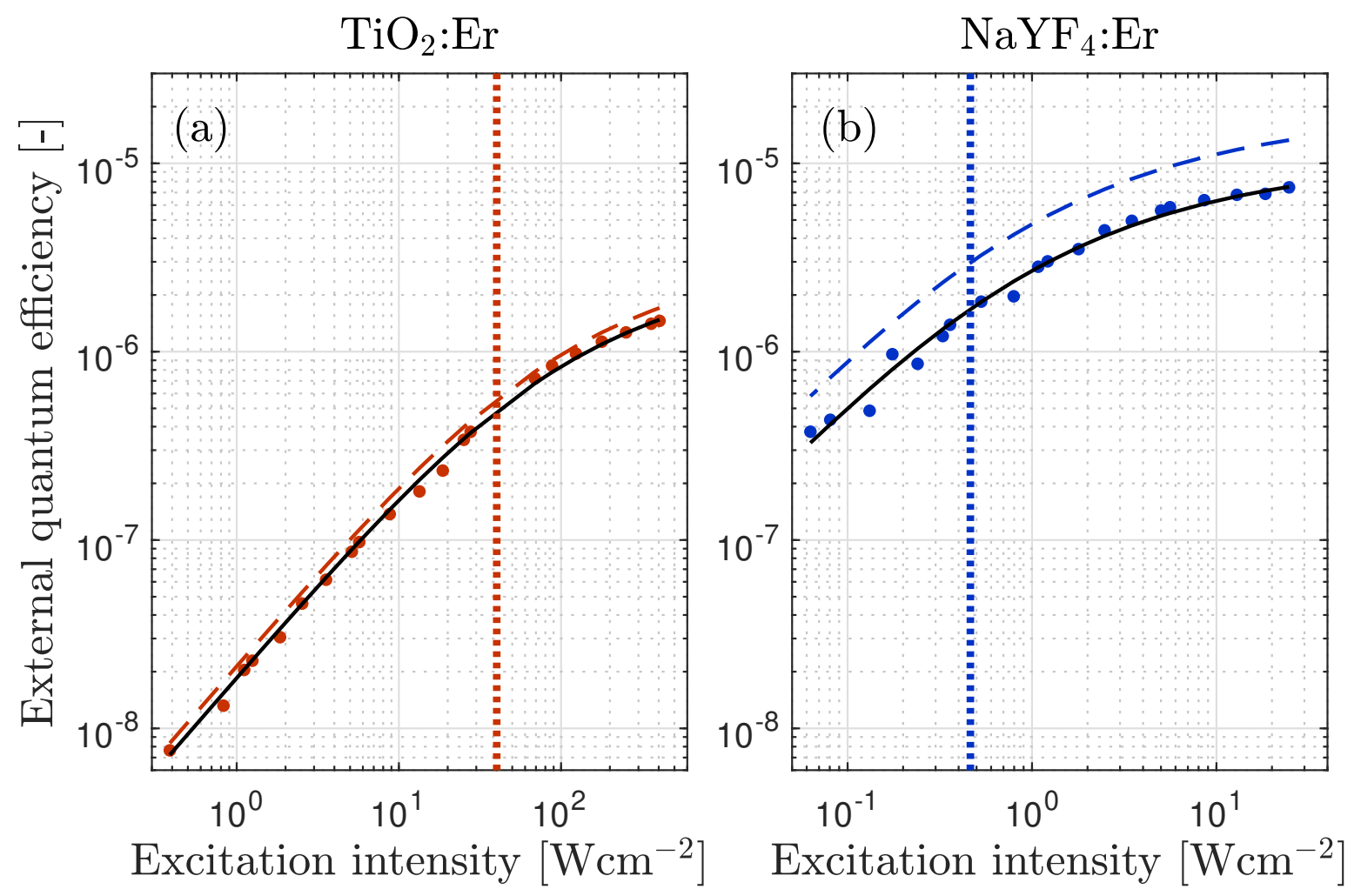

Figure 7. Saturation curves for the $\mathrm{TiO}_{2}$ (a) and $\mathrm{NaYF}_{4}$ (b) upconversion materials, re-scaled to external quantum efficiency using the calibration values from table 1 . The fitted saturation curves (solid black curve) to the measurements (colored data points) are also re-scaled accordingly, and the saturation intensities are indicated by the vertical dashed lines. The predictions of (36) for the external quantum efficiency are plotted as dashed colored curves.

of external quantum efficiency as shown in figure 7. This enables us to perform a consistency check of the general theory discussed in section 2.1. From the measured values stated in table 1 , the absorption cross section $\sigma_{12}$, and the radiative decay constant $A_{31}$ stated in [16], we are able to compute the external quantum efficiency from (36) at arbitrary intensities with $\zeta$ and $C_{\mathrm{ns}}$ set to unity since there is no nanostructures on the surface. The value of $C_{\text {film }}$ is calculated from the definition in (32) using the methods to be explained in section 3. In figure 7, the resulting theoretical curves for the external quantum efficiency for both materials are plotted with dashed curves in the appropriate color coding. A convincingly good agreement is found for both upconverting systems with the theoretical curve, within a factor 2 of the measurements in both cases. This gives a very strong confidence to the entire modeling of section 2.1 and to our estimates of experimental parameters. This includes the assumption of the radiative rates, $A_{21}$ and $A_{31}$, being similar for the two materials, which makes sense to some degree since the radiative decay constant stated in [16] for $\mathrm{NaYF}_{4}$ :Er is already on the level of a pure magnetic-dipole-driven transition and there is no reason to expect this effect to be much different in another material. Taken together, the quantitative understanding of 
Improving the efficiency of upconversion by light concentration using nanoparticle design 20

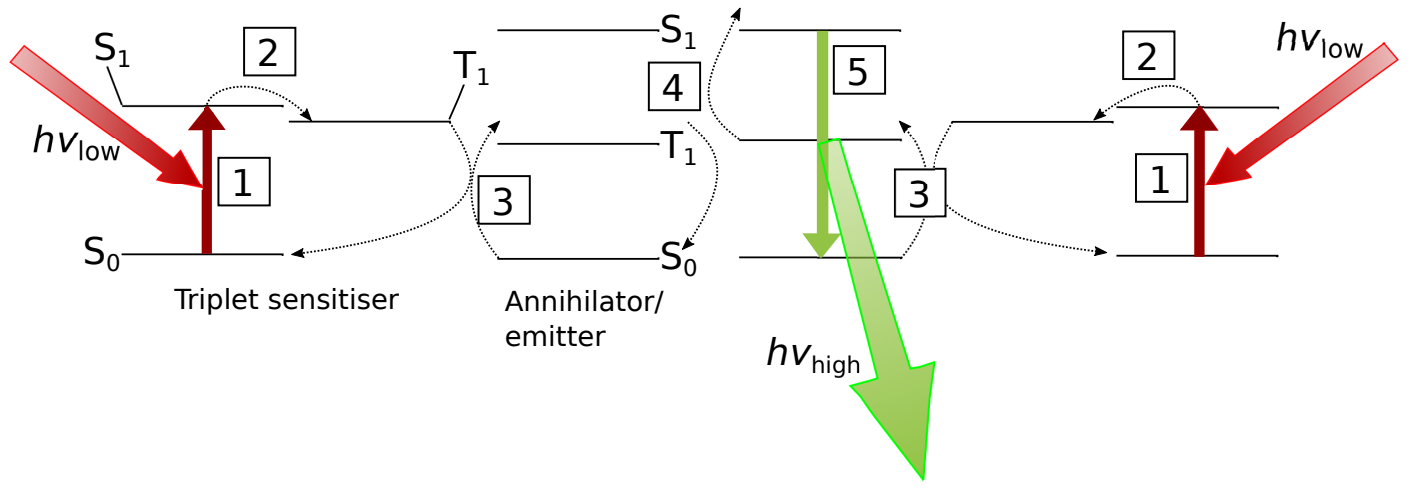

Figure 8. Mechanistic scheme of photon upconversion by sensitized triplet fusion. 1) Excitation of the triplet sensitizer from its singlet ground state $S_{0}$ to its lowest singlet excited state $\mathrm{S}_{1}$ by absorption of a low-energy photon. 2) Intersystem crossing to the lowest triplet excited state $T_{1}$ of the sensitizer. 3) Triplet energy transfer to $T_{1}$ of the triplet annihilater/emitter molecule. 4) Triplet-triplet annihilation, also known as triplet fusion, to produce a singlet excited state $S_{1}$ of the annihilator/emitter. 5) Decay from the $\mathrm{S}_{1}$ to the $\mathrm{S}_{0}$ state of the emitter by emission of a high-energy (upconverted) photon.

the material response to light forms a good starting point for investigating the impact of gold nanostructures for light concentration and enhanced upconversion luminescence.

\subsection{Molecular upconverters}

As an alternative to photon upconverting materials based on lanthanide ions, we have also explored photon upconverting systems based on sensitized triplet fusion among molecular entities [27]. In molecular upconverters, the metastable intermediate state allowing for sequential absorption is decoupled from the absorbing transition which facilitates a combination of strong and broad absorption bands and long-lived intermediate states. These favorable properties have given rise to photon upconversion quantum yields of $38 \%$ in solution under $250 \mathrm{~mW} \mathrm{~cm}^{-2}$ laser diode ( $635 \mathrm{~nm}$ ) excitation [28] and $14 \%$ in a rubbery polymer under 1 sun excitation [29]. The mechanism of upconversion based on triplet fusion is shown in Figure 8.

Lissau et al. showed that green-to-blue photon upconversion via triplet fusion is possible on a nanostructured $\mathrm{ZrO}_{2}$ film cosensitized with triplet sensitizer and annihilator molecules [30]. The upconversion was observable using $536 \mathrm{~nm}$ light with intensities as low as $5 \mathrm{~mW} / \mathrm{cm}^{2}$ which is comparable to the intensity of sunlight. The upconversion signal showed a rise-time of less than $10 \mathrm{~ns}$, suggesting that triplet energy migration via the Dexter mechanism takes place among molecules fixed on the surface. This study showed the potential of implementing a similar system in dye-sensitized solar cells which would increase the theoretical maximum efficiency of these devices from around $30 \%$ to more than $40 \%$ [31]. In a related study with triplet sensitizer and annihilators cochemisorbed on mesoporous $\mathrm{ZrO}_{2}$ film, the photon upconversion efficiency was significantly improved and long-term stability was established with upconverted 
photons observed in samples stored for 1 year under dark ambient atmospheric conditions [32]. The long-term stability was ascribed to a combination of efficient triplet energy migration among annihilators chemisorbed onto the mesoporous surface and efficient photo-induced depletion of molecular oxygen from the mesopores via a photoinduced endoperoxide forming reaction with the polyacene based emitter molecules. Furthermore, systematic variation of individual dye surface coverage showed that the photon upconversion efficiency is limited at high sensitizer surface coverage, where loss mechanisms such as exciton trapping in molecular aggregates and homogenous triplet fusion among triplet sensitizers in separate domains compete with photon upconversion.

An alternative heterogenous architecture was investigated where triplet energy migration and triplet fusion was restricted to a population of triplet annihilators chemisorbed to nanostructured $\mathrm{ZrO}_{2}$ films, while triplet sensitizers were dissolved in a solvent penetrating the mesoporous network of the thin films [33, 34]. Compared to the cosensitized upconverter films the heterogenous systems showed upconversion efficiencies up to three orders of magnitude higher. The higher efficiency was primarily ascribed to triplet sensitization of surface-bound triplet annihilators via molecular diffusion of triplet sensitizers in the bulk solution, which was observed as a strong correlation between the $\mu$ s rise time of the photon upconversion signal and the bulk solution phosphorescence decay time of triplet sensitizers. The Nattestad and Schmidt groups implemented a similar structure in a complete dye-sensitized solar cell [35] as a realization of the molecular intermediate band solar cell suggested by Ekins-Daukes and Schmidt [31]. The power conversion efficiency obtainable from this new solar cell architecture was improved significantly by Hanson and coworkers through development of upconverting self-assembled molecular bilayers on the nanostructured photoanode [36].

While the studies of upconversion on nanostructured films all involve triplet sensitizers containing heavy metal ions for efficient inter system crossing, a more recent study shows the potential of employing a new class of strongly absorbing triplet sensitizers without involvement of heavy metals [37]. In the subphthalocyaninefullerene hybrids dispersion-induced $\pi-\pi$ interactions ensure an efficient "through-space" intramolecular singlet energy transfer while back energy transfer of the generated triplet states and associated energy loss is efficiently inhibited by the lack of orbital overlap between the involved states.

Compared to the lanthanide based photon upconverters the molecular systems for photon upconversion has the additional advantage that the absorbing transition can be tuned by chemical design to absorb throughout the visible part of the electromagnetic spectrum [38]. For applications in solar cells the upconverter should absorb in the farred or near-infrared region. While such molecular upconverters have been designed, their upconversion quantum yields suffer from the energy-gap law [39], restricting the excited state lifetime to values far below the radiative lifetime of the excited state. We have studied rubrene as a material for triplet fusion while using palladium(II) 1,4,8,11,15,18,22,25-octabutoxypthalocyanine $\left(\mathrm{PdPc}(\mathrm{OBu})_{8}\right)$ as a triplet sensitizer. The latter has a $\mathrm{S}_{1} \leftarrow \mathrm{S}_{0}$ Q-band absorption maximum at $725 \mathrm{~nm}$ in toluene solution. This 
Improving the efficiency of upconversion by light concentration using nanoparticle design 22

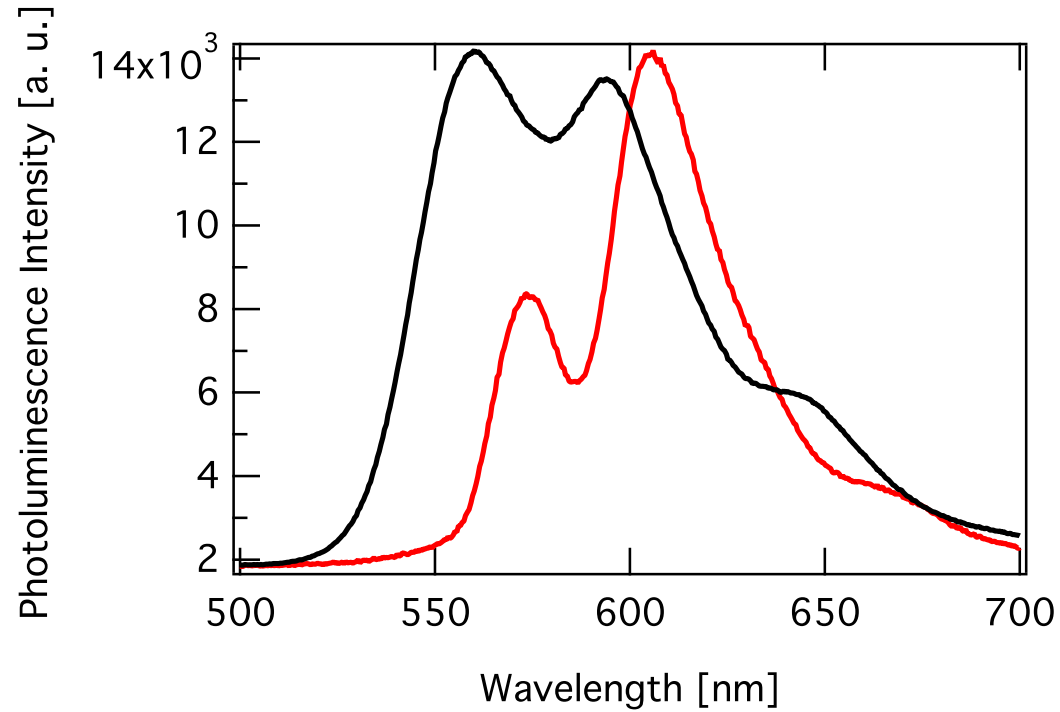

Figure 9. Stokes fluorescence $\left(\lambda_{\text {exc }}=480 \mathrm{~nm}\right)$ from a $\operatorname{PdPc}(\mathrm{OBu})_{8} /$ rubrene pair integrated in a Poly 74-30 rubbery polymer, doped with DBP and sandwiched between protective layers as described by Hagstrom et al.[43]. The black spectrum was recorded at the point of excitation through the lens of a Nikon ME600 fluorescence microscope while the red spectrum was recorded away from the point of excitation.

molecular system was first studied for photon upconversion by Singh-Rachford and Castellano [40] and it is driven by the mechanism shown in Figure 8. The lifetime of the triplet excited state of $\mathrm{PdPc}(\mathrm{OBu})_{8}$ is $3.5 \mu$ s in deaerated solution [41], which is sufficient for triplet sensitisation of the rubrene annihilator for observable photon upconversion in toluene solution [40], in gels [42], and in the ethyleneoxide/epichlorohydrin rubbery polymer [40].

Until now, the highest triplet fusion based photon upconversion quantum yields have been realised in solution phase, but due to the volatility of the applied solvents these systems are not viable for solar energy applications. As potential solid-state host materials for the $\mathrm{PdPc}(\mathrm{OBu})_{8} /$ rubrene upconverter, we have tested two different state-of-the-art rubbery polymers, which are currently the type of solid-state molecular photon upconverters showing the highest photon upconversion quantum yields [44]. The commercially available polyurethanes Poly 74-30 (Polytek) and ClearFlex 50 (Smooth-On) were integrated with the $\mathrm{PdPc}(\mathrm{OBu})_{8} /$ rubrene molecular pair following the procedures published by the Kim, Schmuttenmaer, and Kim groups [43, 45]. Using a $740 \mathrm{~nm}$ pE-100 (CoolLED) light source for excitation and a fluorescence spectrograph (Acton SpectraPro-150) with a charge-coupled device detector (Princeton PIXIS 100) for detection we did not observe a photon upconversion signal in the polyurethane based $\mathrm{PdPc}(\mathrm{OBu})_{8} /$ rubrene systems. For the Poly 74-30 system, samples were studied also including a small fraction of dibenzotetraphenylperiflanthene (DBP) which has previously shown to result in a 19 times increase of photon upconversion signal in a rubrene triplet fusion material [46]. In our studies clear signs of singlet energy transfer from rubrene to DBP was observed as a shift of photoluminescence signal from the 


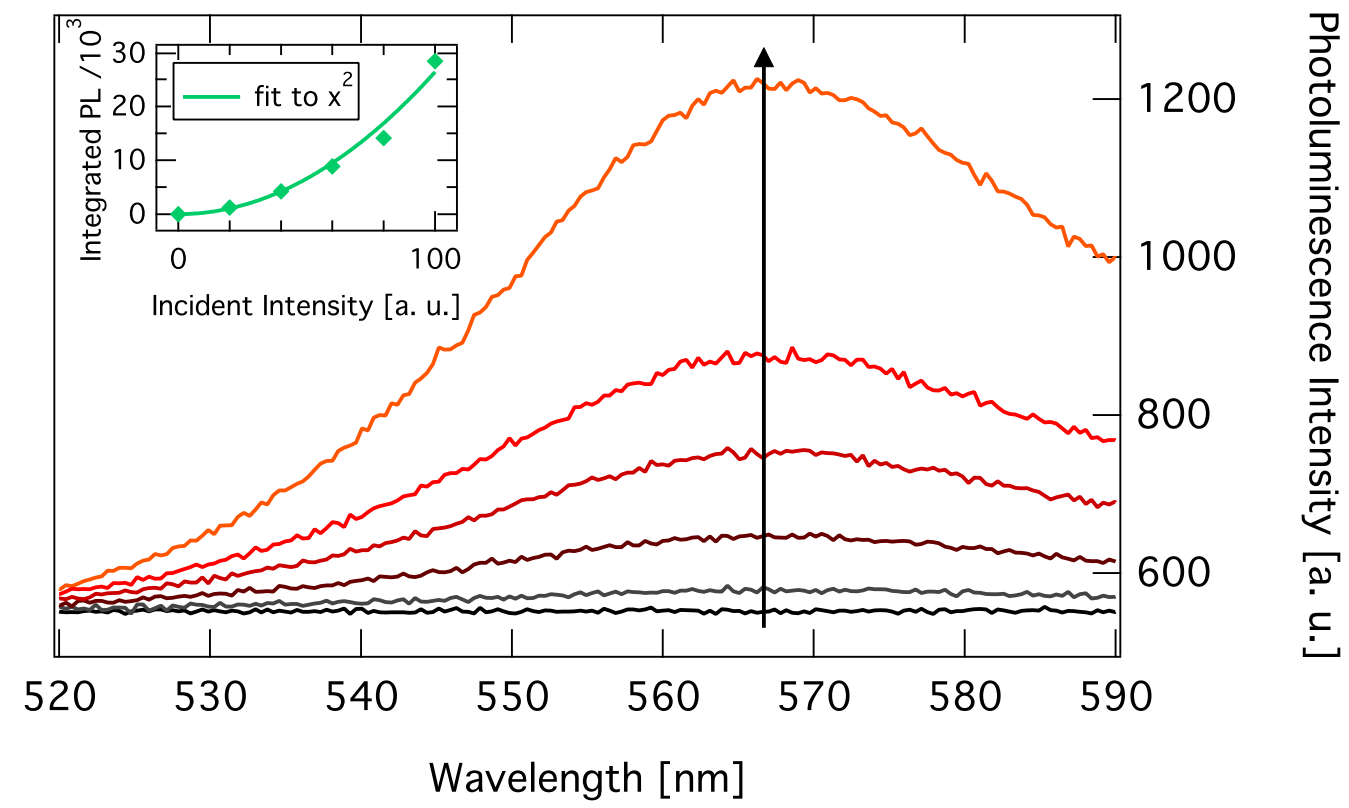

Figure 10. Anti-Stokes fluorescence $\left(\lambda_{\text {exc }}=740 \mathrm{~nm}\right)$ from $\mathrm{PdPc}(\mathrm{OBu})_{8} /$ rubrene on a glass substrate recorded at increasing incident light intensity. The inset shows the background corrected photoluminescence signal, integrated from 520 to $590 \mathrm{~nm}$, as function of incident light intensity. The green line is the best quadratic fit to the data.

fluorescence spectrum of rubrene toward the fluorescence spectrum of DBP as the point of observation was moved away from the point of excitation (Figure 9). However, even with DBP included as an additional emitter, no upconversion signal was detected.

We ascribe the lack of an observable photon upconversion signal in the polyurethane based systems to the relatively short triplet lifetime of the $\operatorname{PdPc}(\mathrm{OBu})_{8} /$ rubrene couple. In previous studies of these rubbery polymers the meso-tetraphenyl-tetrabenzoporphyrin (PdTPBP)/perylene couple was applied as the upconverter. To date this is the molecular upconverter which has shown the highest photon upconversion quantum yield [28]. PdTPBP has a triplet excited state lifetime of ca. $240 \mu \mathrm{s}$ in tetrahydrofuran and in Poly 74-30, i.e. two orders of magnitude higher than the corresponding lifetime for $\mathrm{PdPc}(\mathrm{OBu})_{8}$, which explains why PdTPBP shows efficient triplet energy transfer based on molecular diffusion in a highly viscous solid matrix, where the short triplet lifetime of $\mathrm{PdPc}(\mathrm{OBu})_{8}$ prevents efficient energy transfer. To test this hypothesis we made a sample without the polyurethane matrix by simple drop-casting of a toluene solution of $\mathrm{PdPc}(\mathrm{OBu})_{8}(0.29 \mathrm{mM})$ and rubrene (saturated) onto a glass substrate. Excitation of the sample with $740 \mathrm{~nm}$ light resulted in clearly observable anti-Stokes fluorescence from rubrene, which follows a quadratic power law as function of incident light intensity, as expected for upconversion below the strong annihilation limit [47, 48] (Figure 10). It is remarkable that the upconversion signal was recorded under normal atmosphere where molecular oxygen could be expected to quench the triplet based upconversion mechanism. Instead it seems that the close packing of the molecular component in 


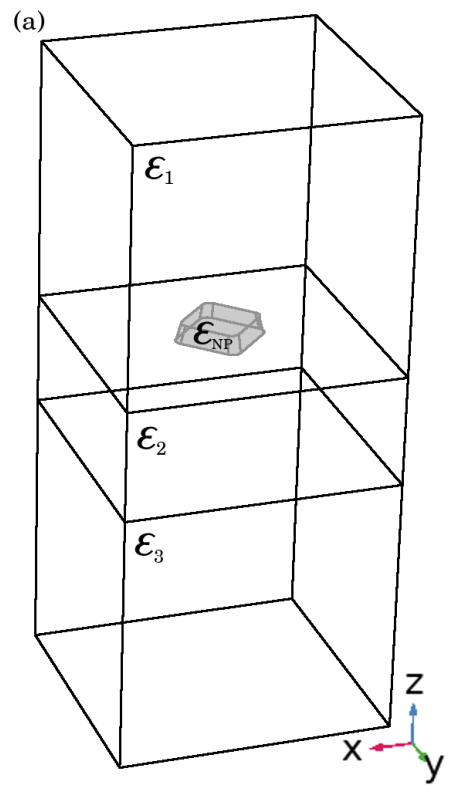

(b)

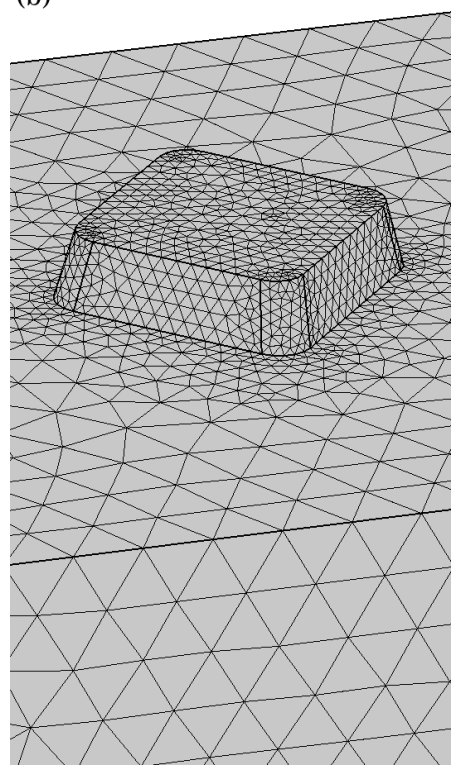

Figure 11. Geometry of a nanoparticle on top of a thin film (a) and zoom-in on a partially meshed geometry (b).

these films facilitates Dexter-type triplet energy migration among physisorbed molecules that is fast enough to outcompete quenching by molecular oxygen. Similar behavior was observed for green-to-blue molecular photon upconverters on nanostructured $\mathrm{ZrO}_{2}$ surfaces $[30,32]$. We are currently performing a more detailed study of the solid-state $\mathrm{PdPc}(\mathrm{OBu})_{8} /$ rubrene system including time-resolved characterization. Furthermore we work towards boosting the efficiency of this system by integrating nanoparticles with broad surface plasmon resonances around the Q-band absorption region of $\operatorname{PdPc}(\mathrm{OBu})_{8}$.

\section{Optical Modeling}

The propagation of electromagnetic waves are described mathematically by Maxwell's equations,

$$
\begin{aligned}
& \nabla \cdot \boldsymbol{D}=\rho_{\mathrm{f}}, \\
& \nabla \cdot \boldsymbol{B}=0, \\
& \nabla \times \boldsymbol{E}=-\frac{\partial \boldsymbol{B}}{\partial t}, \\
& \nabla \times \boldsymbol{H}=\boldsymbol{J}_{\mathrm{f}}+\frac{\partial \boldsymbol{D}}{\partial t},
\end{aligned}
$$

which can be solved to give the spatial and temporal development of the displacement field $\boldsymbol{D}$, the electric field $\boldsymbol{E}$, the magnetic field $\boldsymbol{B}$ and the $\boldsymbol{H}$-field (sometimes also named the magnetic field). In the following section, we will discuss the calculation of the electric field and related properties using these equations. Dipole-sources will 
be related to decay rates and the section will be finished with a discussion of field enhancement. All of this is needed to be able to evaluate and optimize the performance of nano structures to increasing the upconversion yield.

The four vector fields $(\boldsymbol{D}, \boldsymbol{E}, \boldsymbol{B}$, and $\boldsymbol{H})$ in (37)-(40) are related through the constitutive relations for the material. $\rho_{\mathrm{f}}$ and $\boldsymbol{J}_{\mathrm{f}}$ are the "free" charges and currents, not accounted for already in the constitutive relations. The constitutive relations for linear isotropic non-magnetic materials can be written as

$$
\boldsymbol{D}=\epsilon \boldsymbol{E}=\epsilon_{0}\left(\epsilon_{\mathrm{r}}^{\prime}+i \epsilon_{\mathrm{r}}^{\prime \prime}\right) \boldsymbol{E} \quad, \quad \mu_{0} \boldsymbol{H}=\boldsymbol{B},
$$

where $\epsilon=\epsilon_{0} \epsilon_{\mathrm{r}}$ and $\epsilon_{\mathrm{r}}$ is the complex relative permittivity, related to the complex refractive index, $N$, by

$$
N^{2}=(n+\mathrm{i} \kappa)^{2}=\epsilon_{\mathrm{r}}
$$

$\epsilon$ characterizes the response of the electrons in the material to an applied field. If all electrons are tightly bound to the nuclei, the material is an insulator and if some of the electrons can move easily, the material is termed a conductor. An insulator will generally have $\operatorname{Im}\left(\epsilon_{\mathrm{r}}\right)=0$ while conductors have $\operatorname{Im}\left(\epsilon_{\mathrm{r}}\right)>0$.

The time-harmonic ansatz is given by

$$
\begin{array}{lll}
\boldsymbol{E}(\boldsymbol{r}, t)=\boldsymbol{E}(\boldsymbol{r}) \exp (-\mathrm{i} \omega t), & \boldsymbol{B}(\boldsymbol{r}, t)=\boldsymbol{B}(\boldsymbol{r}) \exp (-\mathrm{i} \omega t), \\
\boldsymbol{J}_{\mathrm{f}}(\boldsymbol{r}, t)=\boldsymbol{J}_{\mathrm{f}}(\boldsymbol{r}) \exp (-\mathrm{i} \omega t), & \rho_{\mathrm{f}}(\boldsymbol{r}, t)=\rho_{\mathrm{f}}(\boldsymbol{r}) \exp (-\mathrm{i} \omega t),
\end{array}
$$

and can be inserted into Maxwell's equations to yield the time-harmonic Maxwell's equations, valid for a single frequency $\omega$. Inserting the ansatz, taking the curl of (39) and using (40) result in the time-harmonic wave equation for the electric field,

$$
\nabla \times(\nabla \times \boldsymbol{E})-\omega^{2} \mu_{0} \epsilon \boldsymbol{E}=\mathrm{i} \omega \mu_{0} \boldsymbol{J}_{\mathrm{f}} .
$$

where the field is generated by the external (time-harmonic) current density $\boldsymbol{J}_{\mathrm{f}}$. Note that often $\epsilon=\epsilon(\boldsymbol{r})$ and "defines" the geometry under investigation.

Figure 11(a) shows a typical geometry for scattering calculations, where a nanoparticle $\left(\epsilon=\epsilon_{\mathrm{NP}}\right)$ is placed on top of a thin film (middle, $\epsilon=\epsilon_{2}$ ) on a substrate (bottom, $\epsilon=\epsilon_{3}$ ) embedded in e.g. air (top, $\epsilon=\epsilon_{1}$ ). In this case, it is often more convenient to define $\boldsymbol{J}_{\mathrm{f}}$ implicitly, by assuming that $\boldsymbol{J}_{\mathrm{f}}$ is located outside the region of interest and then split the total field $\boldsymbol{E}$ as

$$
\boldsymbol{E}=\boldsymbol{E}_{\mathrm{b}}+\boldsymbol{E}_{\mathrm{s}}
$$

where $\boldsymbol{E}_{\mathrm{b}}$ is the background field and $\boldsymbol{E}_{\mathrm{s}}$ is the scattered field. $\boldsymbol{E}_{\mathrm{b}}$ is assumed to solve the wave equation without any nanoparticles present, i.e.

$$
\nabla \times\left(\nabla \times \boldsymbol{E}_{\mathrm{b}}\right)-\omega^{2} \mu_{0} \epsilon_{\mathrm{b}} \boldsymbol{E}_{\mathrm{b}}=0 .
$$

where $\epsilon_{\mathrm{b}}=\epsilon$ everywhere except inside the nanoparticle, where $\epsilon_{\mathrm{b}}=\epsilon_{1}$. The scattered field satisfies

$$
\nabla \times\left(\nabla \times \boldsymbol{E}_{\mathrm{s}}\right)-\omega^{2} \mu_{0} \epsilon \boldsymbol{E}_{\mathrm{s}}=-\nabla \times\left(\nabla \times \boldsymbol{E}_{\mathrm{b}}\right)+\omega^{2} \mu_{0} \epsilon \boldsymbol{E}_{\mathrm{b}}
$$


which is to be solved for $\boldsymbol{E}_{\mathrm{s}}$. Note that the right hand side is only non-zero at the positions occupied by the nanoparticles. The background field thus implicitly defines the current density generated by the scatterer, which in turn generates the scattered field. In the case of a stratified background as in figure $11, \boldsymbol{E}_{\mathrm{b}}$ can easily be calculated analytically using the transfer-matrix method. If a more complicated background is needed, a numerical calculation can also be used to obtain the background field.

The finite element method is a general method for solving partial differential equations with possibly complicated geometries [49, 50]. The computational domain is meshed by dividing it into small domains of known and often simple geometry, such as triangles or rectangles in 2D and tetrahedrons or hexahedrals in 3D. An example of this is shown in figure 11(b). Inside each geometric element, the field is approximated using simple analytical expressions, denoted shape functions. The geometric entity together with the types of expressions for the field quantity solved for is known as a finite element. It is important to choose the right shape functions to be able to accurately capture the physics being solved for. In the present case, the electric and magnetic fields must satisfy the Maxwell interface conditions

$$
\begin{aligned}
& \hat{\boldsymbol{n}} \times\left(\boldsymbol{E}_{1}-\boldsymbol{E}_{2}\right)=0 \quad, \hat{\boldsymbol{n}} \cdot\left(\boldsymbol{D}_{1}-\boldsymbol{D}_{2}\right)=\sigma_{\mathrm{f}} \\
& \hat{\boldsymbol{n}} \times\left(\boldsymbol{H}_{1}-\boldsymbol{H}_{2}\right)=\boldsymbol{K}_{\mathrm{f}}, \hat{\boldsymbol{n}} \cdot\left(\boldsymbol{B}_{1}-\boldsymbol{B}_{2}\right)=\sigma
\end{aligned}
$$

where $\hat{\boldsymbol{n}}$ is a unit outward surface normal, $\sigma_{\mathrm{f}}$ is a surface charge density, $\boldsymbol{K}_{\mathrm{f}}$ is a surface current density, and subscript $i, i \in\{1,2\}$, denotes the side of the interface. The conditions state that the tangential component of the electric field must be continuous, while the normal component is allowed to jump over an interface where the permittivity is changing and/or there is a surface charge. To satisfy the interface conditions of the electric field, the degrees of freedom attached to the finite element is the tangential component of the electric field. This type of element is knows as an edge element or a Nedeleć element [49].

Boundary conditions are needed to obtain a unique solution to the electric wave equation and they are also an integral part of the system to be modeled. In many cases the system to be modeled is placed in an open domain, but the computational domain of course has to be finite, and some form of truncation must thus be used. If the system is geometrically periodic and the input is also periodic, periodic boundary conditions can be used to limit the modeling domain to only a single unit cell. Oftentimes the input is only periodic up to a known phase shift and Bloch-Floquet periodic boundary conditions should then be employed, e.g. [49]

$$
\boldsymbol{E}(0, y, z)=\boldsymbol{E}\left(L_{x}, y, z\right) \exp \left(\mathrm{i} k_{x} L_{x}\right),
$$

for a system periodic along the $x$-axis with a unit cell length of $L_{x}$ and an input being a plane wave with wave vector $\boldsymbol{k}=\left(k_{x}, k_{y}, k_{z}\right)^{T}$. If the system has open boundaries they can be truncated with absorbing boundary conditions or by padding the 
modeling domain with an extra layer of fictitious material, known as a perfectly matched layer. The perfectly matched layer is essentially reflection free on the interface to the adjacent domain and absorbing, such that any field entering the perfectly matched layer will be attenuated. The perfectly matched layer is often truncated with some simple boundary condition. Perfectly matched layers are generally better at eliminating fictitious reflections from the boundaries of the computational box than absorbing boundary conditions, but they also require more computational resources [49].

Subsequently, the finite elements are stitched together to make the tangential electric field continuous and to satisfy the electric wave equation (in its weak form) together with the chosen boundary conditions. This results in a large sparse system of linear equations, which can be solved to obtain the unknown degrees of freedom. Together with the chosen elements, the obtained solution of the linear system can be used to reconstruct the electric field in the entire computational domain.

Physical relevant quantities can be calculated from the (numerically calculated) electric field. Assuming the domain shown in figure 11(a) is periodic along the $x$ - and $y$-directions and truncated with absorbing boundary conditions on the top and bottom surfaces and excited by an incoming plane wave with intensity $I_{0}$, the transmission coefficient is calculated by integrating the time-averaged Poynting vector

$$
\langle\boldsymbol{S}\rangle=\lim _{\tau \rightarrow \infty} \frac{1}{\tau} \int_{0}^{\tau} \boldsymbol{S}(\boldsymbol{r}, t) \mathrm{d} t=\frac{1}{2} \operatorname{Re}\left(\boldsymbol{E} \times \boldsymbol{H}^{*}\right)
$$

over a plane surface, $\Omega_{\mathrm{B}}$, near the bottom to get

$$
T=\frac{1}{I_{0}\left|\Omega_{\mathrm{B}}\right|} \int_{\Omega_{\mathrm{B}}}\langle\boldsymbol{S}\rangle \cdot \hat{\boldsymbol{n}} \mathrm{d} A
$$

where $\hat{\boldsymbol{n}}$ is the unit normal to $\Omega_{\mathrm{B}}$ in the $-z$-direction and $\left|\Omega_{\mathrm{B}}\right|$ is the area of $\Omega_{\mathrm{B}}$. Similarly, the reflection coefficient is calculated as

$$
R=1-\frac{1}{I_{0}\left|\Omega_{\mathrm{T}}\right|} \int_{\Omega_{\mathrm{T}}}\langle\boldsymbol{S}\rangle \cdot \hat{\boldsymbol{n}} \mathrm{d} A,
$$

where $\Omega_{\mathrm{T}}$ is a plane surface near the top of the domain with unit normal $\hat{\boldsymbol{n}}$ in the $z$-direction. The scattering cross section can be calculated as

$$
\sigma_{\mathrm{s}}=\frac{1}{I_{0}} \int_{\Omega_{\mathrm{SC}}}\left\langle\boldsymbol{S}_{\mathrm{S}}\right\rangle \cdot \hat{\boldsymbol{n}} \mathrm{d} A=\frac{1}{I_{0}}\left(\int_{\Omega_{\mathrm{T}}}\left\langle\boldsymbol{S}_{\mathrm{s}}\right\rangle \cdot \hat{\boldsymbol{n}} \mathrm{d} A+\int_{\Omega_{\mathrm{B}}}\left\langle\boldsymbol{S}_{\mathrm{s}}\right\rangle \cdot \hat{\boldsymbol{n}} \mathrm{d} A\right),
$$

where $\Omega_{\mathrm{SC}}$ is a surface enclosing the scatterer, $\hat{\boldsymbol{n}}$ is an outward unit normal vector and $\left\langle\boldsymbol{S}_{\mathrm{s}}\right\rangle=\frac{1}{2} \operatorname{Re}\left(\boldsymbol{E}_{\mathrm{s}} \times \boldsymbol{H}_{\mathrm{s}}^{*}\right)$ is the Poynting vector for the scattered field. Note that the first expression in (55) is also valid for non-period structures while the last expression is only valid for periodic systems. Similarly, the absorption coefficient, $A$, and absorption cross section, $\sigma_{\mathrm{a}}$, can be calculated as

$$
\sigma_{\mathrm{a}}=\left|\Omega_{\mathrm{B}}\right| A=\frac{1}{I_{0}} \int_{\mathrm{V}} \omega \epsilon_{0} \epsilon_{\mathrm{r}}^{\prime \prime}|\boldsymbol{E}|^{2} \mathrm{~d} V
$$

where $V$ is the volume of the scatterer. The extinction cross section is the sum of the absorption and scattering cross sections. 
Improving the efficiency of upconversion by light concentration using nanoparticle design 28

The dyadic Green's function, $\stackrel{\leftrightarrow}{\boldsymbol{G}}$, is defined as the dyad satisfying [51]

$$
\nabla \times\left(\nabla \times \stackrel{\leftrightarrow}{\boldsymbol{G}}\left(\boldsymbol{r}, \boldsymbol{r}^{\prime}, \omega\right)\right)-k^{2}(\boldsymbol{r}) \stackrel{\leftrightarrow}{\boldsymbol{G}}\left(\boldsymbol{r}, \boldsymbol{r}^{\prime}, \omega\right)=\stackrel{\leftrightarrow}{\boldsymbol{I}} \delta\left(\boldsymbol{r}-\boldsymbol{r}^{\prime}\right)
$$

where $\overleftrightarrow{\boldsymbol{I}}=\hat{\boldsymbol{x}} \hat{\boldsymbol{x}}+\hat{\boldsymbol{y}} \hat{\boldsymbol{y}}+\hat{\boldsymbol{z}} \hat{\boldsymbol{z}}$ is the unit dyad and $k^{2}(\boldsymbol{r})=\omega^{2} \mu_{0} \epsilon(\boldsymbol{r})$. A particular solution to Eq. (45) is obtained by dotting the definition of the dyadic Green's function from the right with $\mathrm{i} \omega \mu \boldsymbol{J}_{\mathrm{f}}\left(\boldsymbol{r}^{\prime}\right)$, integrate over $\boldsymbol{r}^{\prime}$ and using the wave equation, yielding

$$
\begin{aligned}
& \left(\nabla \times \nabla \times-k^{2}(\boldsymbol{r})\right) \int \stackrel{\leftrightarrow}{\boldsymbol{G}}\left(\boldsymbol{r}, \boldsymbol{r}^{\prime}, \omega\right) \cdot\left(\mathrm{i} \omega \mu \boldsymbol{J}_{\mathrm{f}}\left(\boldsymbol{r}^{\prime}\right)\right) \mathrm{d}^{3} r^{\prime} \\
= & \left(\nabla \times \nabla \times-k^{2}(\boldsymbol{r})\right) \boldsymbol{E}
\end{aligned}
$$

from which the electric field can then be identified as

$$
\boldsymbol{E}(\boldsymbol{r})=\mathrm{i} \omega \int \mu \stackrel{\leftrightarrow}{\boldsymbol{G}}\left(\boldsymbol{r}, \boldsymbol{r}^{\prime}, \omega\right) \cdot \boldsymbol{J}_{\mathrm{f}}\left(\boldsymbol{r}^{\prime}\right) \mathrm{d}^{3} r^{\prime}
$$

It was used that the differential operator can be taken outside the integral. Care must in general be taken with this, as the Green's functions are singular at $\boldsymbol{r}=\boldsymbol{r}^{\prime}$, but if the field is only evaluated outside the source region there is no problem.

To make physical sense out of $\stackrel{\leftrightarrow}{\boldsymbol{G}}$, remember that

$$
\stackrel{\leftrightarrow}{\boldsymbol{G}}=\boldsymbol{G}_{x} \hat{\boldsymbol{x}}+\boldsymbol{G}_{y} \hat{\boldsymbol{y}}+\boldsymbol{G}_{z} \hat{\boldsymbol{z}}
$$

where ${ }^{\wedge}$ means unit vector. Inserting into (57) results in

$$
\begin{aligned}
& \nabla \times\left(\nabla \times \boldsymbol{G}_{x}\right)-k^{2}(\boldsymbol{r}) \boldsymbol{G}_{x}=\hat{\boldsymbol{x}} \delta\left(\boldsymbol{r}-\boldsymbol{r}^{\prime}\right), \\
& \nabla \times\left(\nabla \times \boldsymbol{G}_{y}\right)-k^{2}(\boldsymbol{r}) \boldsymbol{G}_{y}=\hat{\boldsymbol{y}} \delta\left(\boldsymbol{r}-\boldsymbol{r}^{\prime}\right), \\
& \nabla \times\left(\nabla \times \boldsymbol{G}_{z}\right)-k^{2}(\boldsymbol{r}) \boldsymbol{G}_{z}=\hat{\boldsymbol{z}} \delta\left(\boldsymbol{r}-\boldsymbol{r}^{\prime}\right) .
\end{aligned}
$$

The arguments to $\stackrel{\leftrightarrow}{\boldsymbol{G}}$ have been omitted for brevity. Comparing this to the electric field generated by a dipole current density, $\boldsymbol{J}_{\text {Dipole }}=-\mathrm{i} \omega \boldsymbol{p} \delta\left(\boldsymbol{r}-\boldsymbol{r}^{\prime}\right)$ [51]

$$
\nabla \times\left(\nabla \times \boldsymbol{E}_{\text {Dipole }}\right)-k^{2}(\boldsymbol{r}) \boldsymbol{E}_{\text {Dipole }}=\omega^{2} \mu_{0} \boldsymbol{p} \delta\left(\boldsymbol{r}-\boldsymbol{r}^{\prime}\right),
$$

it is seen that each column of $\stackrel{\leftrightarrow}{\boldsymbol{G}}\left(\boldsymbol{r}, \boldsymbol{r}^{\prime}, \omega\right)$ is related to the electric field of a dipole located at $\boldsymbol{r}^{\prime}$ with dipole moment $\boldsymbol{p}$ as

$$
\boldsymbol{E}_{\text {Dipole }}(\boldsymbol{r})=\omega^{2} \mu_{0} \stackrel{\leftrightarrow}{\boldsymbol{G}}\left(\boldsymbol{r}, \boldsymbol{r}^{\prime}, \omega\right) \cdot \boldsymbol{p}
$$

where $\boldsymbol{p}$ is along one of the three axes.

For a two-level quantum system located at $\boldsymbol{r}_{0}$ with transition dipole moment $\boldsymbol{p}=\left\langle\psi_{b}|q \boldsymbol{r}| \psi_{a}\right\rangle$, the decay rate, $\gamma$, can be written as [51]

$$
\gamma=\frac{\pi \omega_{0}}{3 \hbar \epsilon_{0}}|\boldsymbol{p}|^{2} \rho_{\mathrm{p}}\left(\boldsymbol{r}_{0}, \omega_{0}\right)
$$

where $\rho_{\mathrm{p}}$ is the partial local density of optical states, given by

$$
\rho_{\mathrm{p}}\left(\boldsymbol{r}_{0}, \omega_{0}\right)=\frac{6 \omega_{0}}{\pi c^{2}} \hat{\boldsymbol{p}} \cdot \operatorname{Im}\left(\stackrel{\leftrightarrow}{\boldsymbol{G}}\left(\boldsymbol{r}_{0}, \boldsymbol{r}_{0}, \omega_{0}\right)\right) \cdot \hat{\boldsymbol{p}}
$$


A similarly looking expression is obtained by looking at the power emitted by a classical dipole locate at $\boldsymbol{r}_{0}$,

$$
\begin{aligned}
P & =\oint_{\Omega_{\mathrm{D}}}\langle S\rangle \cdot \hat{\boldsymbol{n}} d A=-\frac{1}{2} \int_{\mathrm{V}_{\mathrm{D}}} \operatorname{Re}\left(\boldsymbol{J}_{\text {Dipole }}^{*} \cdot \boldsymbol{E}\right) \mathrm{d} V \\
& =\frac{\omega_{0}}{2} \operatorname{Im}\left(\boldsymbol{p}^{*} \cdot \boldsymbol{E}\left(\boldsymbol{r}_{0}\right)\right)=\frac{\omega_{0}^{3} \mu_{0}}{2} \hat{\boldsymbol{p}} \cdot \operatorname{Im}\left(\stackrel{\leftrightarrow}{\boldsymbol{G}}\left(\boldsymbol{r}_{0}, \boldsymbol{r}_{0}, \omega_{0}\right)\right) \cdot \hat{\boldsymbol{p}} .
\end{aligned}
$$

where $\boldsymbol{p}$ is the classical dipole moment, $\Omega_{\mathrm{D}}$ is a surface enclosing only the dipole and $V_{\mathrm{D}}$ is the volume inside the surface. In the case of free space, one can explicitly calculate the decay rate and the power emitted by a classical dipole and arrive at the conclusion $\gamma / \gamma_{0}=P / P_{0}$ for the relative change in decay rate when the emitter is embedded in an inhomogeneous medium. The power emitted by a dipole near a metallic nanoparticle will induce currents in the metal which will be dissipated as heat. Thus, the power emitted by the classical dipole can further be divided into a part which is absorbed by a metal nanoparticle and a part which is radiated to infinity, $P=P_{\mathrm{R}}+P_{\mathrm{NR}}$. This leads to the expressions for the relative changes in non-radiative and radiative decay rates of the emitter,

$$
\frac{\gamma}{\gamma_{0}}=\frac{P}{P_{0}}=\frac{\gamma_{\mathrm{R}}}{\gamma_{0}}+\frac{\gamma_{\mathrm{NR}}}{\gamma_{0}},
$$

with $\gamma_{\mathrm{R}} / \gamma_{0}=P_{\mathrm{R}} / P_{0}$ and $\gamma_{\mathrm{NR}} / \gamma_{0}=P_{\mathrm{NR}} / P_{0}$. $P_{\mathrm{R}}$ can be calculated as the power transmitted through a surface enclosing the entire system and $P_{N R}$ can be calculated as the power absorbed by the nanoparticle.

The changes in spontaneous radiative and non-radiative decay rates of an atom embedded in an inhomogeneous environment can be calculated by moving a dipole to different locations in space and calculating the power emitted by the dipole, the power radiated to infinity and the power absorbed in metals for all three dipole polarizations [51]. Note that periodic boundary conditions can not be used, as a single dipole breaks the periodicity. In general, a huge number of finite element calculations have to be made (three per dipole position) to get a good spatial resolution of the changes in decay rates. However, using adaptive sampling, the number of calculations can be limited to only the spatial locations where the decay rates are changing, as they will tend towards the homogeneous-space values far from any interfaces. In addition, any symmetries of the geometry can also be used to limit the number of calculations needed. In this way both 2D and 3D maps of the spatial dependency of radiative and non-radiative decay rates can be calculated, even on a modest computer system, as all the calculations are independent [52]. Figure 12 shows such a calculation for a metal nano structure on top of a thin film. The metal structure consists of 4 rectangles arranged as a cross, with no metal in the center. Panel (a) shows the enhancement in the electric field when the structure is excited by a normal-incident plane wave and panel (b) shows the grid where the dipoles are placed. The two lower panels shows the radiative (c) and non-radiative (d) decay rates. It is evident that close to the metal, a high non-radiative decay rate is found, leading to quenching of the upconverted light. 
(a)

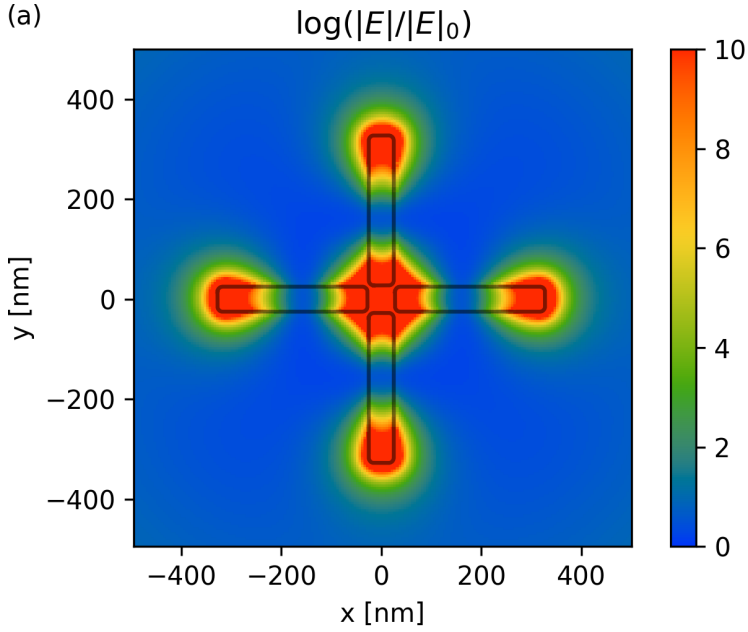

(c)

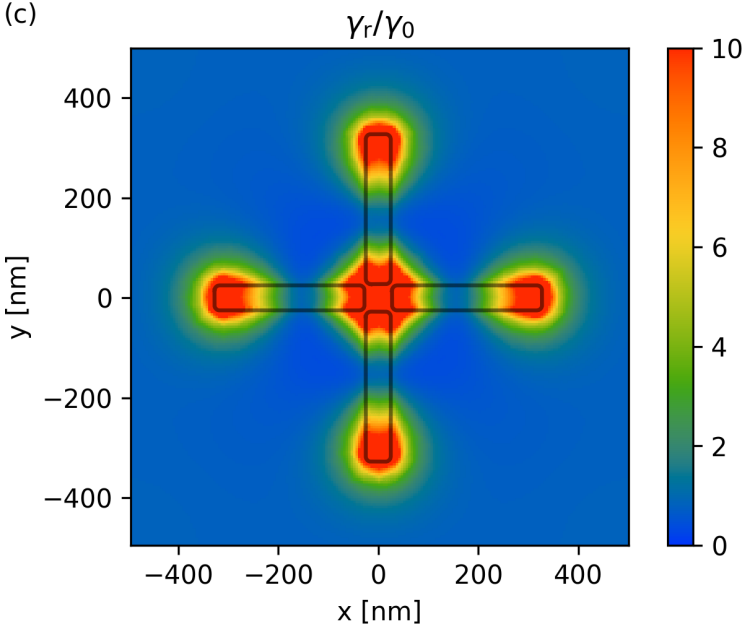

(b)
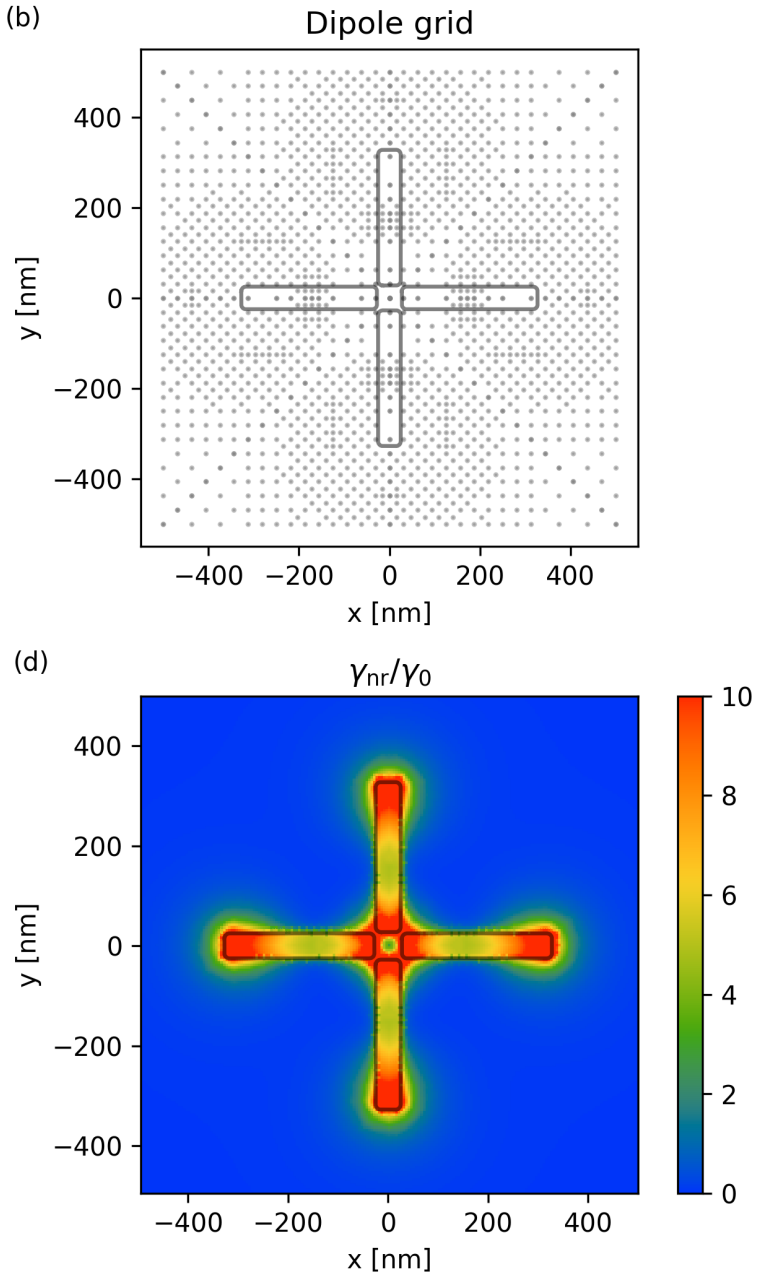

Figure 12. Calculation of decay rates at $\lambda=1558 \mathrm{~nm}$ near four rectangular metal nanoparticles arranged as a cross, with no metal in the center of the cross. Panel (a) shows the electric field enhancement (polarization average), (b) shows the grid used for placing dipoles, (c) shows the radiative decay rates and (d) shows the non-radiative decay rates. The non-radiative decay rate is increased near the particle, where the field is also increased by plasmonic enhancement, leading to quenching of the upconverted light and lower enhancement than expected from the field alone.

\subsection{Field enhancement}

The obvious way of increasing the electromagnetic energy within a region of space is to increase the optical path length. This is in general known as light trapping and has been studied considerably in connection with solar-cell designs, as it is essential for high cell performance. The simplest method to increase the light path in a film is to texture the surface, such that normal incident light will be scattered in multiple directions and thus not go straight through the region where high fields are needed. A backreflector may also be used, such that the light will both enter and exit through the same surface. Under quite general conditions, the maximum enhancement of absorption of light in a medium can be shown to be given by $4 n^{2} / \sin ^{2} \theta$, where $n$ is the refractive index of 
the medium and $\theta$ is half the acceptance cone angle [53]. In [53] Yu et. al. also show that is is possible to beat this limit in the nanophotonic regime, using sub-wavelength structures.

3.1.1. Plasmonics The plasmonic response of the electrons in a metal to an external field can be used to enhance the electric field near a metal nanoparticle. In the case of a sphere with radius much smaller than the wavelength, one can neglect retardation effects and solve for the electric field using an electric potential. The electric potential can be written in terms of Legendre polynomials both inside and outside the small metal nanosphere. By comparing the electric potential outside the particle with the potential of a dipole, the response of of the nanosphere can be described by a dipole with dipole moment of size [54]

$$
p=4 \pi \epsilon \frac{\left(\epsilon_{\mathrm{NP}}-\epsilon\right) a^{3}}{\epsilon_{\mathrm{NP}}+2 \epsilon} E
$$

for a particle with radius $a$, permittivity $\epsilon_{\mathrm{NP}}$ and embedded in a dielectric medium with permittivity $\epsilon$ subjected to a field of magnitude $E$. A plasmonic resonance occurs when the denominator approaches zero, i.e. if $\operatorname{Re}\left(\epsilon_{\mathrm{NP}}\right) \approx-2 \epsilon$. If the material of the nanosphere can be approximated by a simple Drude model [55], Re $\left(\epsilon_{\mathrm{NP}}\right) \approx$ $\epsilon_{0}\left(1-\omega_{\mathrm{p}}^{2} / \omega^{2}\right)$, the resonance condition can be stated as

$$
\omega=\frac{\omega_{\mathrm{p}}}{\sqrt{1+2 \epsilon / \epsilon_{0}}}
$$

where $\omega_{\mathrm{p}}=N \mathrm{e}^{2} /\left(m_{\mathrm{e}} \epsilon_{0}\right)$ is the plasma frequency, $\omega$ the driving frequency, $m_{\mathrm{e}}$ the electron mass and $N$ is the electron density in the material. From these expressions it is seen that the resonance frequency will depend on the material of the particle as well as the material of the surroundings and the magnitude of the resonance also depends on the geometry of the particle. For larger particles of arbitrary shape, numerical methods show that this also hold true and additionally that the resonance frequency is also geometry-dependent [56].

By tuning the geometry of a metal nanoparticle, a dipole-like field of large magnitude (compared to the incoming field) can be created near the nanoparticle, and the resonance wavelength can be adjusted as needed by adjusting the nanoparticle geometry. The localized regions of very high electric field should potentially lead to a large gain in upconversion efficiency but, as was noted above, in the very same regions the non-radiative decay rate is also increased, leading to a much smaller gain in upconversion intensity than expected when considering the field enhancement alone [52].

3.1.2. Waveguiding Waveguides can propagate waves with minimal loss, by confining the waves in one or two dimensions. A slab waveguide is confining the wave in 2D in a thin film surrounded by materials of lower refractive index. In this arrangement, waves in the thin film may be totally reflected and thereby be guided by the slab waveguide. The waveguide supports a set of modes, which can be found by numerical or 
Improving the efficiency of upconversion by light concentration using nanoparticle design32

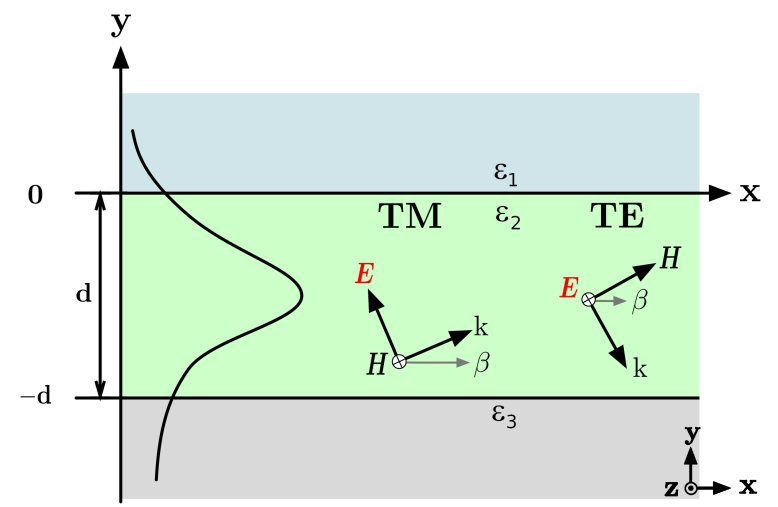

Figure 13. Geometry of a slab waveguide. The curve shown indicate a possible mode profile.

analytical solution of the electric wave equation (45) in the geometry of the waveguide and assuming that the wave is propagating in the thin-film plane, see figure 13. In each of the three layers of the waveguide, the electric field must satisfy the wave equation, and on the interfaces between the layers the interface conditions (49) and (50) must be satisfied. Referring to the coordinate system of figure 13 and assuming that the wave is propagating in the $x$-direction as $\exp (\mathrm{i} \beta x)$, two cases of polarization can be considered. TE polarization is with the electric field having only a component in the $z$ direction and TM polarization is where the magnetic field has only a $z$-component. This leads to

$$
\begin{aligned}
& \frac{\partial^{2} E_{z}^{\mathrm{TE}}}{\partial y^{2}}+\left(k_{0}^{2} \epsilon_{\mathrm{r}}(y)-\beta^{2}\right) E_{z}^{\mathrm{TE}}=0 \\
& \frac{\partial^{2} H_{z}^{\mathrm{TM}}}{\partial y^{2}}+\left(k_{0}^{2} \epsilon_{\mathrm{r}}(y)-\beta^{2}\right) H_{z}^{\mathrm{TM}}=0,
\end{aligned}
$$

with the quantity solved for being continuous in both cases.

The solution in the TE case can be written as

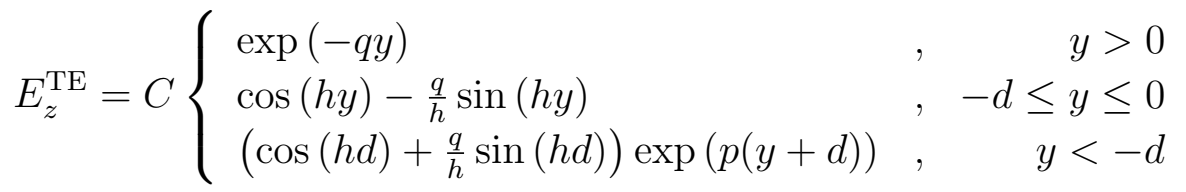

with

$$
q=\sqrt{\beta^{2}-\epsilon_{1} k_{0}^{2}} \quad, \quad h=\sqrt{\epsilon_{2} k_{0}^{2}-\beta^{2}} \quad, \quad p=\sqrt{\beta^{2}-\epsilon_{3} k_{0}^{2}}
$$

where the propagation constant $\beta$ must satisfy the TE mode equation,

$$
\tan (h d)=\frac{p+q}{h\left(1-p q / h^{2}\right)} .
$$

Similar expressions can be obtained for the TM case [57].

One way to excite one or more of the waveguide modes is to use a grating coupler, as sketched in figure 14. A periodic structure on top of a thin film will act as a grating 


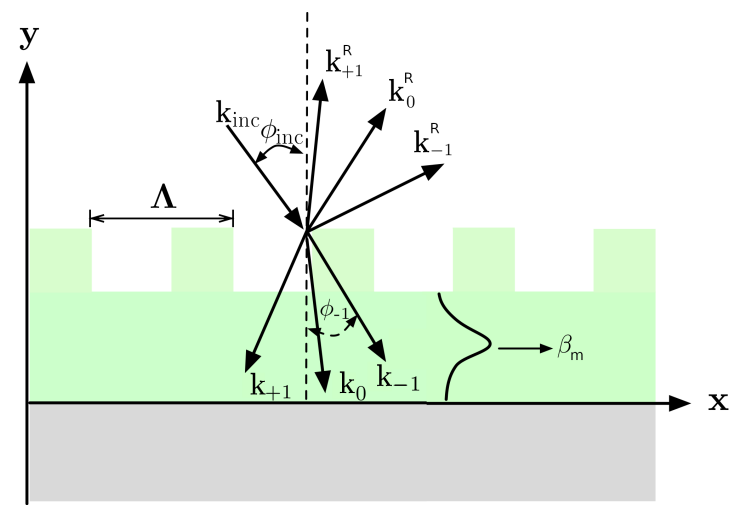

Figure 14. Geometry of a grating coupler where an incoming wave is coupled to a number of diffracted modes in reflection and a number of transmitted modes. The transmitted modes may excite a guided mode in the waveguide.

and diffract an incoming wave into multiple angles. The forward diffracted wave and the incoming wave satisfy the grating equation,

$$
k_{n} \sin \left(\phi_{n}\right)-k_{\mathrm{inc}} \sin \left(\phi_{\mathrm{inc}}\right)=\frac{2 \pi n}{\Lambda}
$$

where $k_{\text {inc }}$ and $k_{n}$ are the magnitudes of the wave vectors of the incoming and forward diffracted waves, respectively, $\Lambda$ is the grating period and $n \in \mathbb{Z}$ is the diffracted wave order. By phase matching one of the forward diffracted waves to one of the guided modes supported by the slab waveguide, an efficient coupling between the incoming light and a wave propagating in the film plane is possible. This happens when $k_{n} \sin \left(\phi_{n}\right)=\beta_{m}$ for a waveguide mode with propagation constant $\beta_{m}$. The phase matching condition

$$
k_{\text {inc }} \sin \left(\phi_{\text {inc }}\right)=\beta_{m}-\frac{2 \pi n}{\Lambda}
$$

can thus be used as a guideline to determine the period $\Lambda$, such that it is possible to get an efficient coupling into a guided mode. Note that there are often many possibilities for phase matching, as there are two polarizations of the incoming light, two polarizations of the guided wave, a number of forward diffraction orders and a number of waveguide modes supported by the film. In addition, even if the phase matching condition is not met exactly, there may still be a coupling to the guided mode [58].

\section{Optimization}

Careful design of plasmonic structures can lead to a substantial enhancement of upconversion [59, 24]. Although intuition and experience may be used to conceive nanoparticle configurations that improve upconversion, the intricate interplay between material properties, geometry features and non-linear nano-optical effects call for systematic inverse design optimization approaches to ensure convergence to (near) optimal solutions. 
Optimization approaches can be divided into sizing optimization, shape optimization and topology optimization [60]. Sizing optimization refers to simple scaling or translation of fixed geometries, c.f. radius and position optimization of pennyshaped nanoparticles. Shape optimization allows more geometrical freedom, by e.g. parameterizing the particle circumference by splines, hence allowing for starfish or more complex shapes [61]. Topology optimization [60] does not require a predefined geometry and hence represents the ultimate design freedom by allowing (dielectric or metallic) material to distribute freely in the design domain. Having originated in mechanical engineering, systematic topology optimization for nanophotonics was initiated at the turn of the millennium for photonic crystal structures [62] and photonic waveguides $[63,64]$. Since then, topology optimization results have inspired general geometric rules for 2D photonic band gap design [65] and it has been applied to a range of applications including: cloaking [66], structural colors [67], extraordinary optical absorption [68], slow light optical waveguides [69], plasmonic effects [70], nanocavities [71], nanoantennas [72], meta surfaces [73, 74] and lately topological insulators [75, 76]. Early work as well as background theory was reviewed in [77] but despite its successes, applications and dissemination has only been spreading slowly to the nanophotonics community. However, recent exposure in high-level journals such as Nature Photonics [78, 79, 80, 81] has respurred interest and the approach is now becoming a general tool in academia as well as industry.

Whether performing sizing, shape or topology optimization, efficient gradient computations using adjoint methods [77, 82, 83] are key to efficient optimization. Nature inspired, non-gradient methods such as genetic algorithms and simulated annealing may be sufficient for problems with few design variables and cheap function evaluations, however, when it comes to complex geometries, expensive modeling and many design variables, such methods are hopelessly inefficient and must be substituted by adjoint sensitivity analysis and mathematical programming-based gradient descent algorithms [84]. A common misconception is that "global approaches" like genetic algorithms and other non-gradient approaches ensure convergence to global minima. This is not true. Such approaches may converge to local minima just as well as gradient-approaches may [84]. Very few optimization problems are convex, and hence all optimization approaches suffer from convergence to local minima. However, in nanophotonics, one can often calculate theoretical maximum performance (like $100 \%$ transmission in a waveguide) and hence, if an optimization process has converged to a design with say 99\% transmission the obtained local minimum may be satisfactory for all practical means. For further discussions on bounds for photonic system performance the reader is referred to [85]. Lately, the increasing popularity of Artificial Intelligence, and deep learning in particular, has lead to a number of publications on the use of such methods for inverse design (see recent review in [86]). Once, such networks have been trained, they may perform quite well on simple geometric design problems. However, considering that tens of thousands of training samples must be used for appropriate training, the computational expense for such approaches are still comparable to genetic algorithms 
Improving the efficiency of upconversion by light concentration using nanoparticle design35

(a)

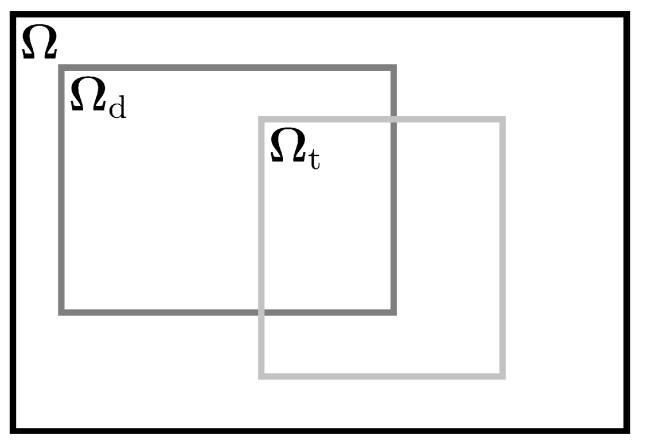

(b)

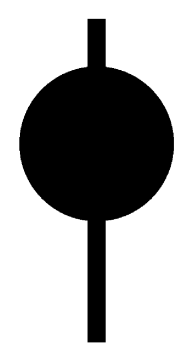

(c)

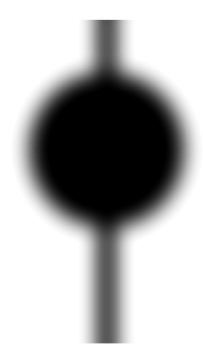

(d)

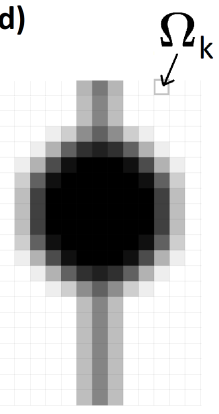

Figure 15. (a) Illustration of the modeling domain $\boldsymbol{\Omega} \in \mathbb{R}^{n}, n \in\{1,2,3\}$ along with the design $\boldsymbol{\Omega}_{\mathrm{d}} \subseteq \boldsymbol{\Omega}$ and target $\boldsymbol{\Omega}_{\mathrm{t}} \subseteq \boldsymbol{\Omega}$ subdomains. (b) Illustration of a discrete spatial material distribution consisting of a solid (black) in a void background (white). (c) Illustration of a continuous material distribution allowing non-physical mixtures of solid and void (gray). (d) Illustration of a model problem spatially discretized into $k$ pixels $\Omega_{\mathrm{k}}$ for the design in (c) used for the numerical modeling.

or other non-gradient approaches and hence still hopelessly inefficient compared to systematic gradient approaches. It is also still an open question if a system trained for a specific physical setup can be used for an alternative physical problem with other boundary conditions and material properties, or if it has to be retrained for these new settings.

In the following, we first outline efficient adjoint sensitivity-based topology optimization in general terms, discussing sensitivity analysis and filtering and thresholding techniques used to promote physically realizable, geometrically robust designs. The outline is followed by a discussion of the method related to our specific applications, as well as a brief discussion of other methods and tools used in the design process.

\subsection{Topology Optimization}

Solving structural design problems using Density Based Topology Optimization (DBTO) is based on numerical simulations of the structure and physics under consideration in a model domain $\boldsymbol{\Omega} \in \mathbb{R}^{n}, n \in\{1,2,3\}$ illustrated in figure 15(a). The structure under design is taken to reside in a subset of $\boldsymbol{\Omega}$, here denoted as the design domain $\boldsymbol{\Omega}_{\mathrm{d}} \subseteq \boldsymbol{\Omega}$. The performance of the structure is measured in another subset of $\boldsymbol{\Omega}$, here denoted as the target domain $\boldsymbol{\Omega}_{\mathrm{t}} \subseteq \boldsymbol{\Omega}$.

When using DBTO to solve a design problem it is formulated as a continuous constrained mathematical optimization problem [87]. The identification of a spatially discrete material distribution constituting the structure is replaced by a continuous material distribution problem, as illustrated in figure 15(b) and 15(c). Here, black denotes the structure and white the background material while grey denotes a nonphysical mixture of the structure and background materials. Allowing non-physical 
mixtures of material in the initial stages of the optimization, enables the use of gradient based optimization methods to solve the design problem. The non-physical mixtures are gradually eliminated in the later stages of the optimization, resulting in physically admissible final designs.

As part of formulating the structural design problem as an optimization problem, the performance of the structure must be measured by an objective function (figure of merit) which is sought minimized or maximized through tailoring the material distribution constituting the structure. Any restrictions on the structure, e.g. the maximally allowed material usage, are formulated as a set of constraints. In its general form the constrained optimization problem may be written as,

$$
\begin{array}{ll}
\max _{\xi} & \Phi\left(\psi_{j}(\xi), \xi\right), \quad j \in\{1,2, \ldots\}, \\
\text { s.t. } & c_{i}\left(\psi_{j}(\xi), \xi\right) \leq 0, \quad i \in\{1,2, \ldots\}, \\
& 0 \leq \xi(\boldsymbol{x}) \leq 1, \quad \forall \boldsymbol{x} \in \boldsymbol{\Omega}_{\mathrm{d}} \quad \boldsymbol{\Omega}_{\mathrm{d}} \subseteq \mathbb{R}^{n}, n \in\{1,2,3\}, \\
& \xi(\boldsymbol{x})=0, \quad \forall \boldsymbol{x} \notin \boldsymbol{\Omega}_{\mathrm{d}}
\end{array}
$$

where $\Phi$ denotes the objective function, which may depend on a set of physical (real or complex) state fields $\psi_{j}=\psi_{j R}+\mathrm{i} \psi_{j I}\left(\psi_{j R}\right.$ and $\psi_{j I}$ being real) as well as on a design field $\xi$ which determines the material distribution constituting the structure under design. In the following only a single state field is considered, i.e. $j=1$. In (82) $c_{i}$ denotes a set of constraints, which may be related to the underlying physical model; to the structural design problem itself; be imposed to achieve a desired effect, etc. Finally, (83) denotes a constraint on the design field, limiting the values it can take throughout the model domain, with $\xi(\boldsymbol{x})=0$ corresponding to one material and $\xi(\boldsymbol{x})=1$ to another. The material distribution in $\boldsymbol{\Omega}$ is determined by $\xi$ and is introduced into the models of the physics through a set of material interpolation functions, $M_{l}\left(\mathcal{M}_{1}, \mathcal{M}_{2}, \xi(\boldsymbol{x})\right), l \in\{1,2, \ldots\}$, which interpolates between parameters for materials $\mathcal{M}_{1}(\xi(\boldsymbol{x})=0)$ and $\mathcal{M}_{2}(\xi(\boldsymbol{x})=1)$.

In all but the most trivial cases no analytical solution exists for (81)-(83). Therefore, the design field and the physical model problem(s) must be discretized in space, using e.g. the finite element method [49], as described in section 3 and illustrated in figure $15(\mathrm{~d})$, and solved to obtain approximate solutions. $\neq$ This discretization results in the design field being represented by $\mathcal{N}_{\xi} \in \mathbb{N}$ continuous design variables. In discretized form the optimization problem may be written as,

$$
\begin{array}{ll}
\max _{\xi_{k}} & \Phi(\psi(\xi), \xi), \quad \xi=\left\{\xi_{1}, \xi_{2}, \ldots \xi_{k}\right\} \\
\text { s.t. } & c_{i}(\psi(\xi), \xi) \leq 0, \quad i \in\{0,1,2, \ldots\}, \\
& 0 \leq \xi_{k} \leq 1, \quad k \in\left\{0,1,2, \ldots, \mathcal{N}_{\xi}\right\},
\end{array}
$$

where the approximation of the state field $\psi(\xi)$ is obtained by solving the corresponding finite element discretized partial differential equation (PDE) modeling the physics,

$$
\boldsymbol{S}\left(M_{l}(\xi)\right) \psi=\boldsymbol{f} .
$$

$\ddagger$ Note that the design variables, $\xi_{k}$, remain continuous. 
Here, $\boldsymbol{S}\left(M_{l}(\xi)\right)$ denotes the system matrix for the governing physics, which depends on $\xi_{k}$ through the material distribution. Assuming a finite element model of the physics, discretizing $\Omega$ into $\mathcal{N}_{\xi}$ elements denoted $\Omega_{k}$, and element-wise constant design variables, the spatial design field may be written in terms of the design variables $\xi_{k}$ as,

$$
\xi(\mathrm{x})=\sum_{k=1}^{\mathcal{N}_{\xi}} \xi_{k} M_{k}(\boldsymbol{x}), \quad M_{k}=\left\{\begin{array}{ll}
1 & \text { for } \boldsymbol{x} \in \Omega_{k} \\
0 & \text { otherwise }
\end{array} .\right.
$$

The change from a discrete to a continuous material distribution problem enables the use of efficient gradient based algorithms such as the globally convergent method of moving asymptotes (GCMMA) [88] to solve the optimization problem. This in turn allows for the solution of design problems involving billions of degrees of freedom (DOFs) [89] rather than a few hundred DOFs which is the feasible limit for non-gradient based approaches. In order to apply gradient based methods such as GCMMA, an efficient approach for computing the gradients $\mathrm{d} \Phi / \mathrm{d} \xi_{k}$ and $\mathrm{d} c_{i} / \mathrm{d} \xi_{k}$, also known as sensitivities, is needed. Adjoint sensitivity analysis [90] offers one such approach. Put briefly, the application of adjoint sensitivity analysis to the discretized optimization problem consists of augmenting the objective function with a set of Lagrange multipliers,

$$
\tilde{\Phi}=\Phi+\lambda_{1}^{\mathcal{T}}(\boldsymbol{S} \psi-\boldsymbol{f})+\lambda_{2}^{\mathcal{T}}\left(\boldsymbol{S}^{*} \psi^{*}-\boldsymbol{f}^{*}\right),
$$

where it is noted that the residue of the discretized state equation from (87) and its complex conjugate are zero. Here $(\cdot)^{\mathcal{T}}$ denotes the transpose and $(\cdot)^{*}$ the complex conjugate. Calculating the total derivative of $\tilde{\Phi}$ with respect to $\xi_{k}$ yields,

$$
\begin{aligned}
\frac{\mathrm{d} \tilde{\Phi}}{\mathrm{d} \xi_{k}}=\frac{\mathrm{d} \Phi}{\mathrm{d} \xi_{k}}= & \frac{\partial \Phi}{\partial \xi_{k}}+\frac{\partial \Phi}{\partial \psi_{R}} \frac{\partial \psi_{R}}{\partial \xi_{k}}+\frac{\partial \Phi}{\partial \psi_{I}} \frac{\partial \psi_{I}}{\partial \xi_{k}} \\
& +\lambda^{\mathcal{T}}\left(\frac{\partial \boldsymbol{S}}{\partial \xi_{k}} \psi_{R}+\boldsymbol{S} \frac{\partial \psi_{R}}{\partial \xi_{k}}+\mathrm{i} \frac{\partial \boldsymbol{S}}{\partial \xi_{k}} \psi_{I}+\mathrm{i} \boldsymbol{S} \frac{\partial \psi_{I}}{\partial \xi_{k}}-\frac{\partial \boldsymbol{f}}{\partial \xi_{k}}\right) \\
& +\lambda^{\mathcal{T}, *}\left(\frac{\partial \boldsymbol{S}^{*}}{\partial \xi_{k}} \psi_{R}+\boldsymbol{S}^{*} \frac{\partial \psi_{R}}{\partial \xi_{k}}-\mathrm{i} \frac{\partial \boldsymbol{S}^{*}}{\partial \xi_{k}} \psi_{I}-\mathrm{i} \boldsymbol{S}^{*} \frac{\partial \psi_{I}}{\partial \xi_{k}}-\frac{\partial \boldsymbol{f}^{*}}{\partial \xi_{k}}\right),
\end{aligned}
$$

which, when collecting terms and defining the adjoint problem

$$
\boldsymbol{S} \lambda=-\frac{1}{2}\left(\frac{\partial \Phi}{\partial \psi_{R}}-\mathrm{i} \frac{\partial \Phi}{\partial \psi_{I}}\right)^{\mathcal{T}}
$$

reduces to

$$
\frac{\mathrm{d} \Phi}{\mathrm{d} \xi_{k}}=\frac{\partial \Phi}{\partial \xi_{k}}+2 \operatorname{Re}\left(\lambda^{\mathcal{T}}\left[\frac{\partial \boldsymbol{S}}{\partial \xi_{k}} \psi-\frac{\partial \boldsymbol{f}}{\partial \xi_{k}}\right]\right),
$$

where Re denotes the real part and it was used that $\lambda_{1}=\lambda_{2}^{*}=\lambda$. This approach eliminates the prohibitively expensive step of computing the derivative of the state field with respect to each design variable $\xi_{k}$ individually, using e.g. finite differences, by instead solving a single adjoint problem (91) of identical size to the original state problem (87). A similar procedure may be used to compute the sensitivities of each constraint, $c_{i}$, depending on the state field. 
Improving the efficiency of upconversion by light concentration using nanoparticle design38

(a)

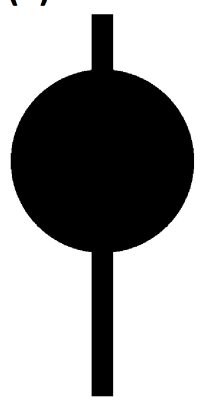

(b)

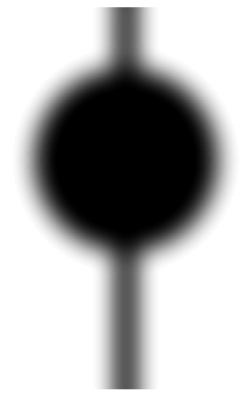

(c)

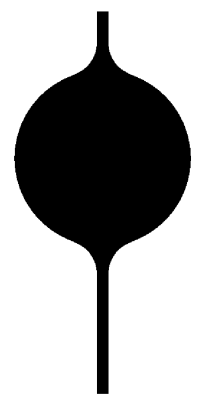

(d)

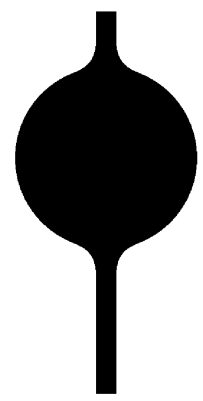

(e)

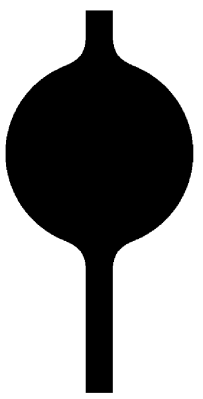

Figure 16. Illustration of the effect of the smoothing filter (93) and threshold operation (95) on the design field, $\xi$. (a) Design field, $\xi$. (b) Smoothed field, $\tilde{\xi}$. (c) Eroded field, $\overline{\tilde{\xi}}^{\eta_{1}=0.6}$, (d) nominal field, $\overline{\tilde{\xi}}^{\eta_{2}=0.5}$, (e) dilated field, $\overline{\tilde{\xi}}^{\eta_{3}=0.4}$.

While the procedure outlined above allows for the solution of the structural design problem, the resulting design field is likely to contain a significant fraction of the design variables $\xi_{k}$ 's taking non-physical values between 0 and 1 . Further, no length-scale is imposed in the design. Thus, the final solution is likely to be non-physical and even if that is not the case, it may be impossible to realize the design using present day available fabrication techniques. Fortunately, resolving these issues has been the focus of a significant research effort [91, 92, 93, 94, 95, 96].

Originating in DBTO for problems in linear elasticity [91, 92], density filtering is a way of regularizing the optimization problem and a step in introducing length-scale in the design [93]. Density filtering may be performed by applying the following volume preserving operator to the design field

$$
\tilde{\xi}\left(\boldsymbol{x}_{i}\right)=\frac{\int_{\Omega_{\mathrm{d}}} w\left(\boldsymbol{x}_{i}-\boldsymbol{x}_{j}\right) \xi\left(\boldsymbol{x}_{j}\right) \mathrm{d} \boldsymbol{x}_{j}}{\int_{\Omega_{\mathrm{d}}} w\left(\boldsymbol{x}_{i}-\boldsymbol{x}_{j}\right) \mathrm{d} \boldsymbol{x}_{j}}, \quad w(\boldsymbol{x})= \begin{cases}R-|\boldsymbol{x}| & \forall|\boldsymbol{x}| \leq R \\ 0 & \text { otherwise }\end{cases}
$$

This operation introduces a length-scale into the design problem controlled by the filter radius $R$. An alternate approach for performing the filter operation in (93), proposed in [97], is to solve an auxiliary PDE in $\boldsymbol{\Omega}_{\mathrm{d}}$ with zero Neumann boundary conditions given as

$$
r^{2} \nabla^{2} \tilde{\xi}+\tilde{\xi}=\xi, \boldsymbol{x} \in \boldsymbol{\Omega}_{\mathrm{d}}, \quad \frac{\partial \tilde{\xi}}{\partial \boldsymbol{n}}=0, \boldsymbol{x} \in \boldsymbol{\Gamma}_{\mathrm{d}},
$$

where $\boldsymbol{\Gamma}_{\mathrm{d}}$ denotes the boundary of $\boldsymbol{\Omega}_{\mathrm{d}}$. Using this approach the filter radius is controlled by $r=R / 2 \sqrt{3}$. Figure 16(a) and 16(b) provides an illustration of the application of the smoothing filter.

In order to promote physically admissible final designs a widely used strategy is to apply the following threshold operation to $\tilde{\xi}\left(\boldsymbol{x}_{i}\right)$ coupled with a continuation procedure for the thresholding strength [93, 94, 95],

$$
\overline{\tilde{\xi}}\left(\boldsymbol{x}_{i}\right)=\frac{\tanh (\beta \eta)+\tanh \left(\beta\left(\tilde{\xi}\left(\boldsymbol{x}_{i}\right)-\eta\right)\right)}{\tanh (\beta \eta)+\tanh (\beta(1-\eta))}, \eta \in[0,1], \beta \in \mathbb{R}^{+} .
$$


Here, $\eta$ denotes the threshold level and $\beta$ the threshold strength. The continuation procedure is applied in the process of solving the optimization problem and consists of gradually increasing $\beta$ from 1 to a high enough value that $\overline{\tilde{\xi}}$ almost exclusively takes values of 0 and 1 for all $k$. Hereby, only minor post-processing is required to arrive at the final physically admissible design. An illustration of the result of applying the threshold procedure for $\eta \in\{0.6,0.5,0.4\}$ and $\beta=1000$ to $\tilde{\xi}$ in figure 16 (b) is shown in figure 16(c)-16(e).

When applying the filter and threshold procedure, the material parameters are interpolated using $\tilde{\xi}\left(\boldsymbol{x}_{i}\right)$. This introduces modifications of the sensitivities which may be computed trivially using the chain rule as

$$
\frac{\mathrm{d} \Phi}{\mathrm{d} \xi_{k}}=\frac{\mathrm{d} \Phi}{\mathrm{d} \Phi} \frac{\mathrm{d} \tilde{\tilde{\xi}}}{\mathrm{d} \tilde{\xi}} \frac{\mathrm{d} \tilde{\xi}}{\mathrm{d} \xi_{k}}, \quad k \in\left\{1,2, \ldots, \mathcal{N}_{\xi}\right\} .
$$

The application of (95) destroys the length-scale introduced by (93), however it is possible to reintroduce length-scale along with a degree of geometric robustness towards near-uniform perturbations by applying the robust approach proposed in [95] and extended in [96] with an introduction of a double filtering procedure. Considering the approach from [95] for simplicity, the approach consists of solving the optimization problem for three different realizations of $\tilde{\xi}$ obtained by choosing different $\eta$-values for each realization, which ideally corresponds to near-uniform erosion/dilation of the design, as illustrated in figure 16(c)-16(e). When applying the robust approach and/or when optimizing for multiple values of other parameters, such as wavelength, the optimization problem may be written as a max-min problem,

$$
\begin{array}{ll}
\max _{\xi_{k}} & \min _{\eta_{p}, \lambda_{m}}\left(\Phi\left(\psi_{p}\left(\overline{\tilde{\xi}}^{\eta_{p}}, \lambda_{m}\right), \overline{\tilde{\xi}}^{\eta_{p}}\right)\right), \quad p \in\{1,2,3\}, \quad m \in \mathbb{N}^{+} \\
\text {s.t. } & c_{i}\left(\psi_{p}\left(\overline{\tilde{\xi}}^{\eta}, \lambda_{m}\right), \overline{\tilde{\xi}}^{\eta_{p}}\right) \leq 0, \quad i \in\{0,1,2, \ldots\}, \\
& 0 \leq \xi_{k} \leq 1, \quad k \in\{0,1,2, \ldots\} .
\end{array}
$$

Although the min function in (97) is non-differentiable, the problem can be transformed into an equivalent continuous optimization problem by introducing an extra variable, acting as the lower bound on the min function, and turning the arguments of the min function into constraints, thereby allowing the use of gradient information [88].

The cost of applying the robust approach is that three state problems (and corresponding adjoint problems) must be solved,

$$
\boldsymbol{S}\left(M_{l}\left(\overline{\tilde{\xi}}^{\eta_{p}}\right) \psi_{p}=\boldsymbol{f}, \quad p \in\{1,2,3\} .\right.
$$

\subsection{Design of nanostructures}

The UCL yield is calculated by use of (29) with the $f^{(\mathrm{b})}$ function used to represent the experimental condition of a Gaussian laser beam. The UCL enhancement can then be 
computed as the ratio of the yields on and off the nanostructures as

$$
L_{\mathrm{UCL}}=\frac{Y_{\mathrm{UCL}}^{\mathrm{on}}}{Y_{\mathrm{UCL}}^{\mathrm{off}}}=\frac{\int_{\mathcal{V}_{\mathrm{UC}}} f^{(\mathrm{b})}\left(\frac{|\boldsymbol{E}|^{2}}{\left|E_{\mathrm{sat}}\right|^{2}}\right) \mathrm{d} V}{\int_{\mathcal{V}_{\mathrm{UC}}} f^{(\mathrm{b})}\left(\frac{\left|\boldsymbol{E}_{b}\right|^{2}}{\left|E_{\mathrm{sat}}\right|^{2}}\right) \mathrm{d} V},
$$

where $|\boldsymbol{E}|^{2}$ is the simulated electric field with the nanostructure present on the film surface, $\left|\boldsymbol{E}_{b}\right|$ is the "background" electric field without nanostructures on the surface, and $\left|\boldsymbol{E}_{\text {sat }}\right|^{2}$ is the background electric field at the experimental conditions (polarization and angle of incidence) of the intensity-dependence measurement, where $I_{\text {sat }}$ was determined. The argument of the $f$-function is scaled by the excitation-level factor $\bar{I} / I_{\text {sat }}$ to the desired intensity, see [19] for a thorough derivation. To apply this in DBTO, the choice of an incoming intensity must be made. A simpler form can be derived such that it is capturing the physics in both the high and the low excitation regime, by expanding the integrands around an intermediate intensity $\sim 10 I_{\text {sat }}$, which is valid for a large range of intensities as can be seen from figure 4a. This leads to a cubic dependence on the electric field. Calculating the average of both polarizations, the objective function becomes

$$
\Phi=\frac{1}{2} \sum_{\mathrm{s}, \mathrm{p}} \frac{\int_{\Omega_{\mathrm{t}}} g(\boldsymbol{x}, \overline{\tilde{\xi}}(\boldsymbol{x}))|\boldsymbol{E}(\boldsymbol{x}, \overline{\tilde{\xi}}(\boldsymbol{x}))|^{3} \mathrm{~d} \boldsymbol{x}}{\int_{\Omega_{\mathrm{t}}}|\boldsymbol{E}(\boldsymbol{x}, \overline{\tilde{\xi}}(\boldsymbol{x})=0)|^{3} \mathrm{~d} \boldsymbol{x}}, \quad n \in \mathbb{N}^{+},
$$

where the sum is taken to average the enhancement over $s$ - and $p$-polarized incident light and $g(\boldsymbol{x}, \overline{\tilde{\xi}}(\boldsymbol{x}))$ is included to enable limiting of the spatial region in which the contribution from the integral in the numerator is included [98]. Hereby one may exclude the enhancement of $|\boldsymbol{E}|^{3}$ near Au-surfaces, not contributing to the upconversion process due to the quenching phenomenon.

The electric field in (102) is obtained by solving discretized versions (87) of the model PDE for either the full electric field (45) or the scattered field (48) with an excitation consisting of a linearly polarized incident plane wave.

The material distribution in the design domain is determined through $\overline{\tilde{\xi}}$ using the interpolation scheme proposed in [99],

$$
\begin{aligned}
\epsilon_{\mathrm{r}}(n(\overline{\tilde{\xi}}), \kappa(\overline{\tilde{\xi}})) & =\left(n(\overline{\tilde{\xi}})^{2}-\kappa(\overline{\tilde{\xi}})^{2}\right)-\mathrm{i}(2 n(\overline{\tilde{\xi}}) \kappa(\overline{\tilde{\xi}})) \\
n(\overline{\tilde{\xi}}) & =n_{\mathcal{M}_{1}}+\overline{\tilde{\xi}}\left(n_{\mathcal{M}_{2}}-n_{\mathcal{M}_{1}}\right) \\
\kappa(\overline{\tilde{\xi}}) & =\kappa_{\mathcal{M}_{1}}+\overline{\tilde{\xi}}\left(\kappa_{\mathcal{M}_{2}}-\kappa_{\mathcal{M}_{1}}\right) .
\end{aligned}
$$

This scheme was demonstrated to possess several beneficial properties, resulting in a near monotonic increase of $\Phi$ during the solution of the optimization problem as well as leading to highly performing designs.

An outline of the full design procedure may be stated as follows. The initial configuration for the model, design and target domains is found using parameter sweeps for basic geometrical parameters such as cell sizes and layer thicknesses. Based on these studies, DBTO is applied to generate highly optimized, but sometimes 
geometrically complex nanoparticle layouts. These geometries can directly be used in e.g. electron beam lithography using proximity-effect-correction software, see section 5.1.1. Sometimes it is, however, desirable to simplify and post-optimize through sizing and shape optimization before manufacturing. In these cases, the objective function was often found to only change slightly even though the number of design variables were reduced significantly to allow for the use of e.g. a Nelder-Mead simplex algorithm to perform fine tuning of the geometry through shape optimization. The approach was able to generate structures which remain highly efficient, while solely consisting of shapes resembling well-known geometric forms.

\section{Fabrication and characterization of nanoparticle designs}

Over the years, the field of micro- and nanofabrication has continuously evolved, confronting the challenges of improving resolution, increasing throughput, and reducing fabrication costs. Some of these fabrication tools, which are used both in research and industry, are electron beam lithography (EBL), photolithography, and nanoimprint lithography (NIL). In this section, we focus on EBL and NIL because of their ability to produce small complex nanoscale features. A proximity-effect-correction (PEC) scheme that helps to boost the resolution of the EBL is described. The section ends with a brief review on two experimental techniques for characterizing the optical response of nanostructures.

\subsection{Electron beam lithography}

In EBL, an electron beam is scanned across the surface of a thin sacrificial layer of electron-sensitive resist, which allows for selective removal of exposed (unexposed) areas in a subsequent development step for positive (negative) resists. The small focus (typically a few nanometers) of the electron beam allows for the production of highresolution structures. However, it is ultimately limited by forward scattering of the electrons traveling through the resist and the backscattering of the electrons from the underlying substrate. The length scale of the backscattering is in the order of tenths of $\mu \mathrm{m}$, whereas the forward scattering of the electrons is a local effect in the range of nanometers [100]. These effects result in exposure of the resist not only in the intended areas but also in areas nearby, thus distorting the original design. This effect is known as the proximity effect. A simple approach to limit proximity effects is to increase the electron's kinetic energy and decrease the resist thickness. This will reduce the beam widening from forward scattering in the resist as well as reducing the local

backscattering from the substrate. However, in order to increase the resolution of the fabricated structures, prepossessing of the intended design has to be performed.

5.1.1. Proximity-effect-correction For high-resolution EBL it is required to correct for proximity effect; early proximity-effect-correction (PEC) schemes date back to the 1970s 
[101]. The three principal PEC schemes can be classified as dose correction, pattern size compensation, and background exposure compensation [102]. Dose correction is done by modifying the electron-beam write pattern and exposure at different locations in the resist to account for exposure smearing resulting from electron scattering. PEC schemes in this group include the self-consistent method [101, 103], the pattern-area density map [104], and different transformation- [105] and optimization-based schemes [106, 107, 108]. Pattern size compensation schemes employ a more empirical approach where the pattern shapes are modified according to experimentally-determined design tables and work best for relatively simple designs [109, 110]. The third method for PEC relies on background exposure to compensate the non-uniform electron energy distribution from backscattering, and is traditionally named GHOST [111]. Furthermore, various hybrid approaches exist $[112,113,114]$ as well as geometry assisted PEC design rules [115] and minimal variation of critical-development time [116].

The PEC scheme utilized by us, namely dose regularization via filtering and projection [117], is part of the latter category. This method uses an image-based representation of the dose pattern and formulates the PEC scheme as an optimization problem. In order to achieve a two-toned dose pattern, a dose regularization strategy is applied which is based on filtering and projection techniques developed in the field of topology optimization already described in section 4.1. This tool has been made available as an open-source Python package on pypi under the package name pecpy [118].

The mathematical reasoning is described in [117] and is briefly introduced here. Assuming that the resist layer is thin allows for neglecting any dependence of the exposure E on the distance from the resist surface to the substrate [119]. The modeling is then simplified to two-dimensional space where the effective dose $\boldsymbol{x}^{\mathrm{E}}$ is given as the incoming dose convoluted by a point spread function (PSF) that accounts for the electron-scattering effects,

$$
\boldsymbol{x}^{\mathrm{E}}=\boldsymbol{x}^{\mathrm{D}} * \mathrm{PSF} .
$$

* denotes here convolution and is similar to the operation in (93) with $w$ being the PSF. In general, when both forward and backward scattering of the electrons are considered, a sum of two Gaussians of different widths is used [111]. However, the focus of this PEC scheme is forward scattering and thus the PSF is chosen as a single Gaussian with variance $\sigma^{2}$ expressed via a beam-broadening parameter $\alpha=\sqrt{2} \sigma$.

Development of the exposed resist is essentially removal of areas with exposure below a threshold value $\eta$ (a positive resist is assumed, and the PEC scheme has only been formulated for such). The development process is modeled by applying an elementwise Heaviside step function $H_{\eta}$ yielding the desired physical material distribution

$$
\boldsymbol{x}^{\mathrm{P}}=H_{\eta}\left(\boldsymbol{x}^{\mathrm{D}} * \mathrm{PSF}\right)
$$

$H_{\eta}$ is numerically used in a smoothed version, similar to the right hands side of (95) such that gradients can be obtained using adjoint sensitivity analysis as in section 4.1. Obtaining the dose pattern that yields physical structures equal to the design 

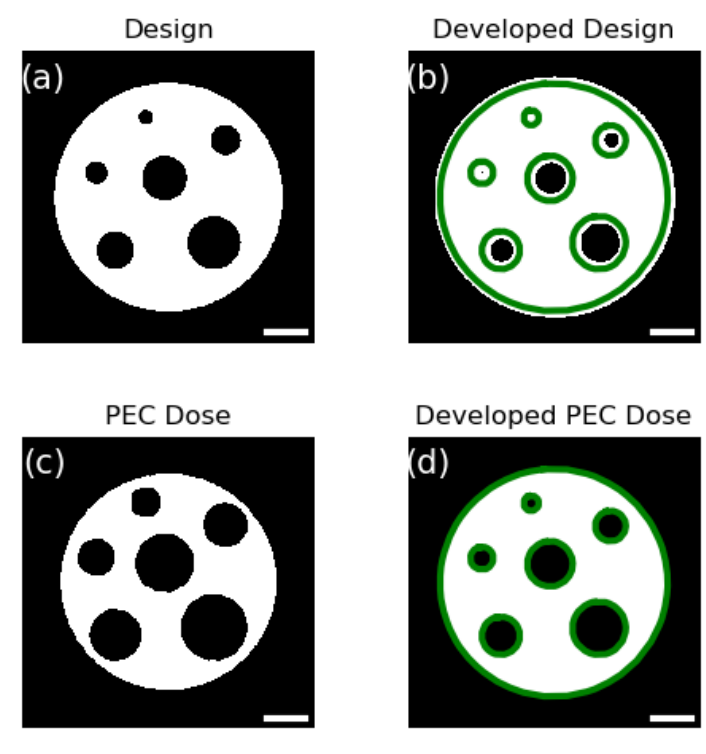

Figure 17. Schematic illustration of the effect of applying PEC. Panel (a) is the design of interest. Panel (b) illustrates the achieved physical structure if panel (a) is used directly as input for the EBL process. Panel (c) illustrates a calculated PEC dose using $(\eta, \alpha)=(0.12,20 \mathrm{~nm})$. Panel $(\mathrm{d})$ is the corresponding developed physical structure. The intended design is imposed as a green outline. The scale bar in each panel is $100 \mathrm{~nm}$.

can be stated as a least-squares optimization problem, efficiently solved by gradient based optimization methods.

The effect of implementing PEC is illustrated in figure 17 where it is evident that using the design directly as the dose pattern results in a broadening of features - in this specific case, closing of the holes in the disc. In contrast, for the calculated PEC dose pattern (panel c)), an excellent match between the developed and intended design is achieved.

5.1.2. PEC parameter determination The physical dose parameters, $\eta$ and $\alpha$, depend on all aspects of the EBL process such as substrate material, resist type and thickness, acceleration voltage and current of the electron beam, the development process, etc. However, they do not depend on the chosen design, which makes it possible to use the same parameters for arbitrary designs as long as the EBL process parameters are kept constant.

In order to determine $\eta$ and $\alpha$, a two-dimensional array of PEC-corrected designs, each design associated with a unique value of $(\eta, \alpha)$, is fabricated. A schematic example is given in figure 18 where the intended design is the one shown in panel a) of figure 17. Following the full structure fabrication process, the resulting array is inspected using SEM to determine the structure most accurately representing the desired design, thereby identifying the dose parameters $(\eta, \alpha)$ of the EBL process. Such an SEM investigation can be seen in figure 18. For this demonstration, the structures were fabricated in $\mathrm{Au}$ 

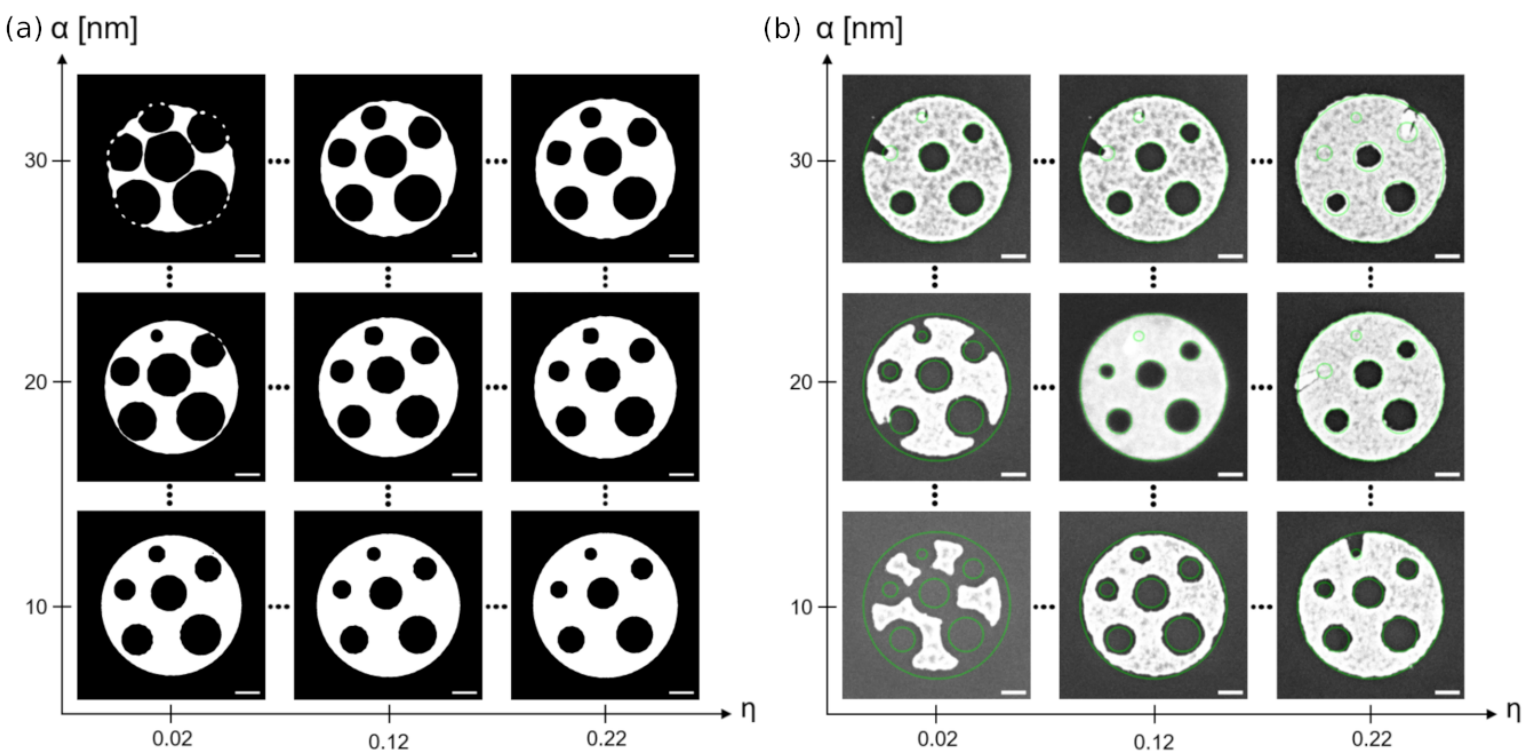

Figure 18. Panel (a) shows an array consisting of calculated PEC dose patterns using different values of $\eta$ and $\alpha$. Panel (b) shows an array consisting of SEM images for structures corrected using different values of $\eta$ and $\alpha$. The intended design is imposed as a green outline, clearly demonstrating that the optimal parameter choice is $(\eta, \alpha)$ $=(0.12,20 \mathrm{~nm})$ corresponding to the middle panel. The scale bar in each panel is 100 nm. Figure adapted with permission from [117].

on a Si substrate. For details about the fabrication, please refer to ref. [117].

The pecpy package is capable of performing PEC for both a single structure and periodic structures. Since the correction is performed on the Fourier transform, the correction is per default performed for periodic structures. In this case, the PEC domain should correspond to the unit cell of the periodic structure. In the case of a single isolated structure, a padding of empty space surrounding the structure is included in the PEC calculations to suppress the effects from periodicity which are included by default through the Fourier transform.

\subsection{Nanoimprint lithography}

Although EBL is an excellent tool for investigation and optimization of nanostructure designs, the throughput and cost is a hurdle for large-scale production, especially when structures with high nanostructure density and complexities are to be produced. In contrast, NIL is a simple and cost-effective tool for mass production with high resolution and throughput. NIL involves a reusable mold to imprint the elevated features by physical deformation of a curable resist, coated on the targeted wafers.

The material properties of the resist is one of the critical parameters which impact the resolution as well as the throughput of NIL. After coating the resist on a substrate, it is heated above the glass transition temperature of the resist in order to reduce its elastic modulus. Under suitable pressures, the mold with (elevated) patterns is pressed 
into the resist (molten phase) so that the resist flows and fills up the cavities of the mold. Subsequently the resist is cooled down to retain its normal phase (hardened) before the mold is removed, resulting in the resist-patterns on the substrate which are the inverted imprints of the mold. Usually molds are coated with an anti-sticking layer, which facilitates the removal of the mold and increases its re-usability. After the imprint, in addition to the achieved resist-patterns, a layer of residual resist typically remains on the compressed regions on the substrate. The residual resist can be removed, for example, by using reactive ion etching. Once the mask (patterned resist) is prepared, the structures can be realized either by etching or metallization of the substrate through the mask.

The above-mentioned process is also defined as Thermal-NIL, whereas it is known as UV-NIL if the type of used resist is photo-curable. Nevertheless, the principle of imprinting remains the same in both cases. Furthermore, NIL can be sub-grouped as i) plate-to-plate ii) roll-to-plate and iii) roll-to-roll NIL, based upon the arrangements of mold and targeted substrate. The types of NIL, and its pros and cons can be studied in detail elsewhere $[120,121,122]$.

A good quality 2D-array of gold nanodiscs fabricated on a silicon substrates, using plate-to-plate Thermal-NIL, is presented in figure 19. The substrate was cleaned with acetone and isopropanol, and dried afterwards with nitrogen blow. The substrate was spin-coated with (thermoplastic) nanoimprint resist (Nanonex nxr-1025) at $2800 \mathrm{rpm}$, providing a film thickness of $190 \mathrm{~nm}$. The sample was baked at $150{ }^{\circ} \mathrm{C}$ for 4 minutes on a hot plate. The imprint was carried out at temperature and pressure values of $170{ }^{\circ} \mathrm{C}$ and $180 \mathrm{psi}$, respectively. A patterned mold with round pillars (230 nm diameter, $200 \mathrm{~nm}$ height), arranged in hexagonal pattern, was used in the Nanonex-NIL system. The $38 \mathrm{~nm}$ layer of residual resist was removed using oxygen plasma. After the mask preparation, $50 \mathrm{~nm}$ layer of gold was deposited by using e-beam evaporation. Lift-off was carried out to strip the unwanted gold, leaving $50 \mathrm{~nm}$ heigh gold nanodiscs on top of the Si substrate (figure 19).

\subsection{Characterization}

Once the nanostructures have been fabricated, the next step is to characterize them optically to test that they perform as expected. In the following, we describe two experimental method that we find useful in this regard. The first method is based on measuring transmission and extinction, which easily reveals the far-field resonance frequencies of the nanostructure. The measurements, if properly calibrated, are also useful for quantitatively benchmarking simulated extinctions. The second method utilizes ultrashort laser pulses to write contours of the nanostructure's electric near-field enhancements (NFEs) in a photosensitive resist. This approach provides a stringent and direct check of electric-near-field simulations, which is particularly useful in this work where the near fields are utilized directly for enhanced upconversion in erbium. 


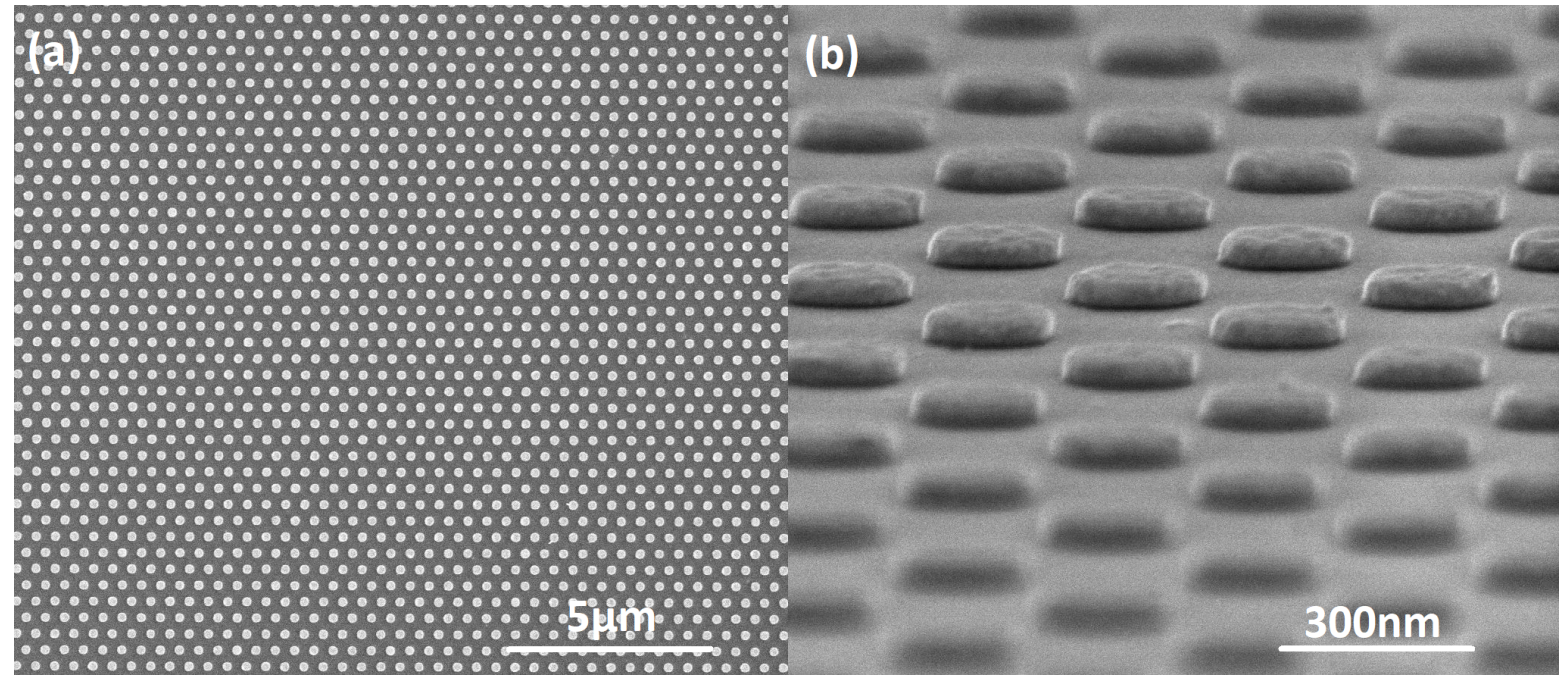

Figure 19. SEM images of fabricated Au nanodiscs on the Si substrate using NIL tool. (a) Top view, (b) tilted view.

5.3.1. Transmission and extinction Different definitions of extinction may be found in the literature [123]. From an experimental point of view, the following definition is convenient: Suppose that a parallel, monochromatic beam of finite size illuminates a region containing the nanostructure. Extinction $E$ is then the ratio between the incident power $P_{\mathrm{i}}$ and the power removed in the forward direction; the latter is just the incident power minus the direct-transmitted power $P_{\mathrm{t}}$. Thus,

$$
E \equiv\left(P_{\mathrm{i}}-P_{\mathrm{t}}\right) / P_{\mathrm{i}}
$$

By "direct transmission" we mean the total flux transmitted in the same direction as that of the incident beam in absence of the nanostructure.

The rationale behind (108) is quite simple: light rays incident upon the nanostructure either pass through unhindered with proportion $T$ (i.e. they are directly transmitted), or else they are partly absorbed, and partly scattered into other directions. Extinction encompasses both absorption and scattering such that $E+T=1$. The meaning of absorption is obvious, but scattering is more ambiguous. Here, as just noted, we understand scattering as a redirection of the incident energy flux. However, in conventional theoretical expositions [124], scattering is defined in terms of the flux of the scattered electromagnetic field which is defined with respect to some reference field (typically the incident or background field, see section 3). The definition is somewhat arbitrary, and experimentally it is awkward because it is always the response due to the total fields that is measured. Nevertheless, in the conventional approach, extinction is also due to total scattering and absorption, but because of a different definition of scattering, the results of such calculations will generally not agree with the experimentalbased definition in 108 as we illustrate by an example below.

Figure 20(a) shows a simple procedure for measuring extinction [c.f. (108)]: The sample containing the nanostructure is placed in the path of a well-collimated beam 


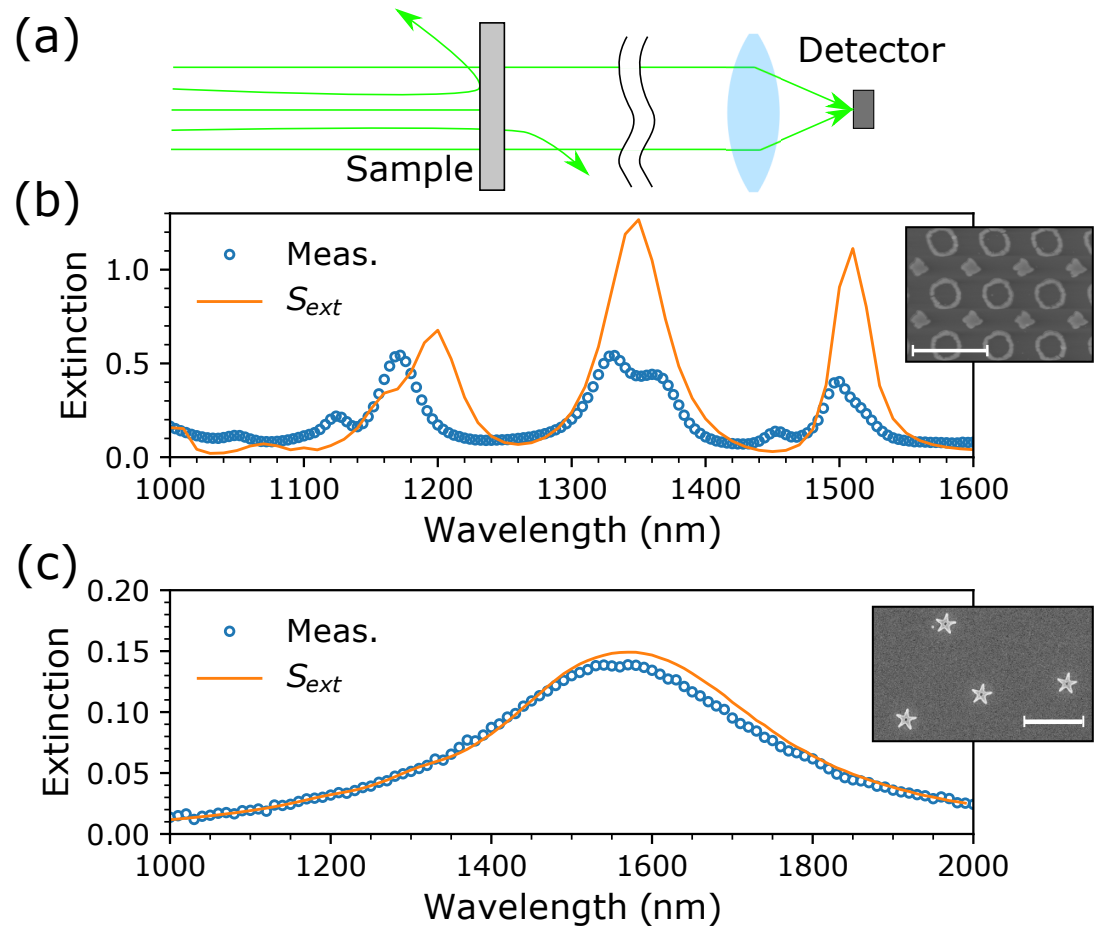

Figure 20. (a) Illustration of an extinction measurement: parallel light rays are incident from the left upon the sample which scatters and absorbs a portion of the light rays. The direct-transmitted rays are imaged onto a photodetector. (b) An example of an extinction measurement (blue circles) of a periodic array of nanoparticles placed on an Er-doped $\mathrm{TiO}_{2}$ film. The inset displays an SEM image of the nanoparticles (the scale bar is $1 \mu \mathrm{m}$ ). The solid orange line (labeled " $S_{\text {ext }}$ ") is a calculation. Extinction is here due to the nanoparticles only, and does not include contributions from the substrate. (c) Extinction measurements and calculations of randomly distributed nanoparticle, shown in the right inset (the scale bar is $1 \mu \mathrm{m}$ ).

of light while a photodetector on the right records the direct-transmitted power. It is important that the photodetector subtends a small solid angle as viewed from the sample plane in order not to capture any scattered light rays (otherwise, extinction is underestimated due to a larger measured power). This can be achieved quite easily by placing the detector far away from the sample.

Typically, the nanoparticles are placed on a substrate or immersed in solution which may contribute to an additional unwanted extinction $E_{\mathrm{b}}$. ( $E_{\mathrm{b}}$ is straightforward to obtain from a reference sample or solution containing no nanoparticles.) To get the extinction $E_{\mathrm{NP}}$ due to the nanoparticles or nanostructure, one simply subtracts $E_{\mathrm{b}}$ from the total extinction $E$ : $E_{\mathrm{NP}}=E-E_{\mathrm{b}}$.

Panel (b) of figure 20 show a comparison between measured (blue circles) and calculated (solid line) polarization-averaged extinction for a periodic arrangement of EBL-produced nanoparticles on top of a Er: $\mathrm{TiO}_{2}$ thin film (an SEM image of the structure is displayed in the inset). The calculation (labeled " $S_{\text {ext }}$ ") is based on the conventional approach where $E$ is calculated from the extinction Poynting vector, 
obtained by decomposing the total electric field into an incident and a scattered part [124]. The agreement for this nanostructure is seen to be only qualitative. This is to be expected, since the calculation is not immediately related to the experimental measurements of direct-transmitted powers. The latter is slightly more difficult to compute because it demands a far-field transformation of the simulated fields in order to classify the resulting poynting vector according to direction. Still, the calculation generally does agree well with the location of the extinction peaks. Note that some peak splitting is seen in the measurements. We attribute this to imperfections in the periodicity in the two directions, which differed on average by approximately $10 \mathrm{~nm}$.

It is interesting to note that the $S_{\text {ext }}$ calculation predicts $E>1$ around $1350 \mathrm{~nm}$ and $1500 \mathrm{~nm}$. This is an example of the extinction paradox [124] where it appears that more power is depleted from the incident beam by the nanostructure than was directed towards it. Such high extinctions can of course never be observed experimentally. The issue is that the $S_{\text {ext }}$ computation generally does not correspond to an observable quantity because of the way in which the electromagnetic fields were arbitrarily decomposed into a background field and a scattered field.

Nevertheless, the extinction computed by the $S_{\text {ext }}$ method can be shown - under simplifying circumstances, such as for isolated nanoparticles - to agree with (108) [124]. Indeed, we have on several occasions obtained very decent quantitative agreement with extinction measurements on randomly-distributed nanoparticles [23, 125], which at least approximately behaves as isolated nanoparticles. An example is shown in figure 20 (c). The extinction calculation was performed on a single nanoparticle in a finite volume surrounded by absorbing boundary conditions (PMLs). The calculated extinction was then scaled according to the average occupied area per nanoparticles of the fabricated structure.

To summarize, the conventional approach to calculating extinction based on the extinction Poynting vector compares quantitatively well with the experimental definition (108) for randomly-distributed nanoparticles. But when inter-particle couplings are strong, as in the case of periodic nanostructures, the computational predictions can only be used qualitatively, the reasons of which we pointed out. To compare quantitatively to (108), one would have to directly calculate the direct transmission (with and without nanoparticles), which is numerically slightly more involved as it requires a far-field transformation of the calculated electromagnetic field in the computational volume.

\subsubsection{Electric near-field mapping Direct measurements of the electric-field amplitudes} surrounding a nanostructure or a nanoparticle is useful for validating electric near-field calculations: in this project, we need to be sure that the near fields are as predicted by simulations, and thereby that the enhanced erbium-upconversion is in fact due to the near field enhancements. However, direct measurements of near fields is challenging because a resolution well-below the diffraction limit is required. One approach is to use scanning near-field optical microscopy techniques where a nanometer-sized probe interacts with the nanostructure. The major issue is, however, that it is the interaction 
between probe and nanostructrue that is imaged, and thus not the electric fields inherent to the unperturbed nanostructure.

A different approach using femtosecond laser pulse excitation was proposed in [126]. It relies on the fact that the onset of laser ablation (material removal) appears only above a well-defined threshold of laser fluence (pulse energy per unit area). In [126], nanoparticles were placed on a silicon substrate and irradiated below the ablation threshold of silicon; local ablation around the nanoparticles was observed, which provided proof of increased electric fields (hence absorption) surrounding the particles.

In fact, if both the local incident fluence $F$ and the threshold fluence $F_{\text {th }}$ is known, it can be inferred that the local fluence must have been enhanced by the nanoparticles by the ratio $F_{\mathrm{th}} / F$. Since fluence scales as the square of the electric field, the near-field enhancement (NFE) can be estimated as

$$
\mathrm{NFE}=\sqrt{F_{\mathrm{th}} / F} .
$$

Using this approach, contours of near-field enhancements can be mapped out by ablation and compared directly to the simulated near-field patterns $[127,128,125]$. The approach is however not restricted to ablation; any permanent change in the medium surrounding the nanoparticles that possess a threshold onset, such as optical phase change $[129,130]$ or photopolymerization $[131,132]$, can be used.

We recently described our progress on near-field mapping in a previous paper [25]. Currently we are pursuing an approach like in [132] where the nanoparticles are embedded in the photoresit SU8; upon sufficient exposure to laser irradiation above a threshold (which now depends on the number of pulses), parts of the photoresist polymerizes. The unpolymerized SU8 is washed away (developed) in a subsequent step, whereafter only the above-threshold exposed SU8 remains which can be imaged by SEM and atomic force microscropy (AFM).

The near-field mapping procedure using SU8 is exemplified in figure 21. We fabricated a random array of nanoparticle crosses with legs of length $200 \mathrm{~nm}$ and height $50 \mathrm{~nm}$, containing a small $40 \mathrm{~nm}$ gap in the center. The nanoparticles were placed on a $\mathrm{TiO}_{2}$ film, and were embedded in $450 \mathrm{~nm}$ SU8. From extinction measurements described in Section 5.3.1, we found a broad resonance around $1700 \mathrm{~nm}$. Therefore, the samples were irradiated with $1700 \mathrm{~nm}$ fs pulses (spectral FWHM $\sim 60 \mathrm{~nm}$ ). In this case we used 10,000 pulses with an average peak fluence around $170 \mathrm{~mJ} \mathrm{~cm}^{-2}$. The radial distribution of the beam's laser fluence follows a Gaussian with a spot size of 8.2(3) $\mathrm{\mu m}$ at $1 / e^{2}$. Panel (a) depicts a topographic atomic force microscope (AFM) image of such a laser spot wherein a central polymerized disc (boundary indicated by the green line) remains because the exposure in the center of the beam exceeded the polymerization threshold of SU8.

Panel (b) shows a closer look at an area about $17 \mu \mathrm{m}$ away from the central spot where four nanoparticles are seen; the upper left nanoparticle contains no visible amounts of SU8, but the remaining three do. The green arrow indicates the direction toward the center of the laser spot (i.e. increasing fluence); the upper-right yellow arrows 

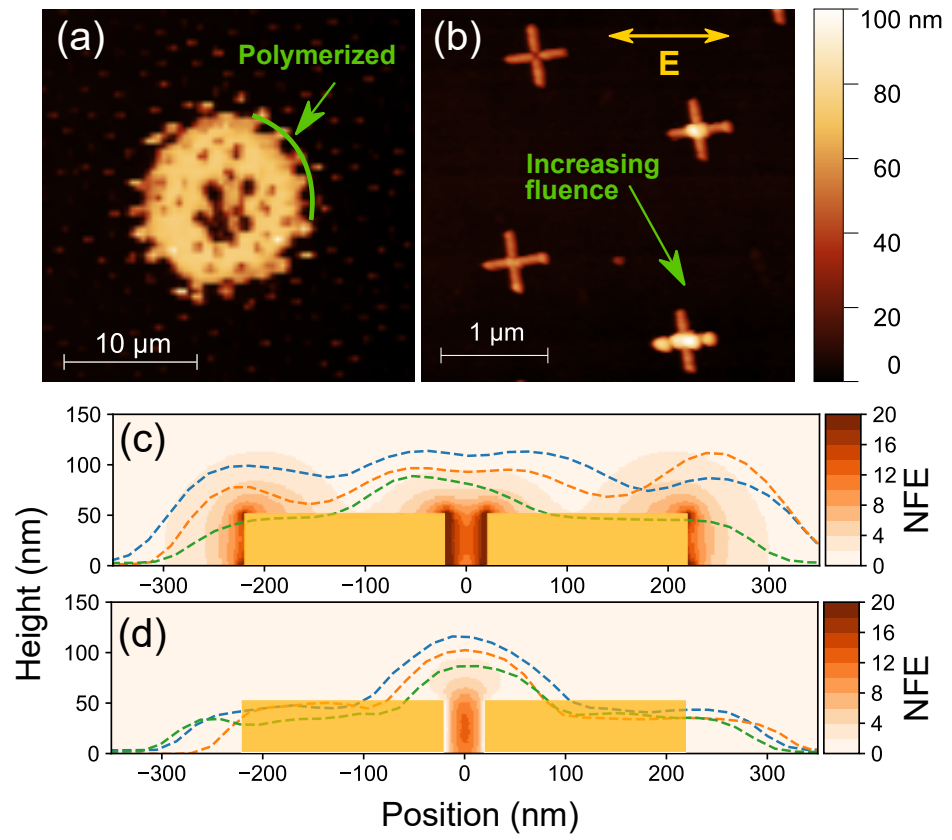

Figure 21. Laser exposure of nanoparticles embedded in SU8 which has subsequently been developed. (a) Area exposed to 10,000 laser pulses. The laser fluence exceeded the polymerization threshold of bare SU8; the marked green outline shows the position where the local exposure equals the polymerization threshold. (b) Region outside the polymerized central spot, showing SU8 sticking to the nanoparticles in the direction of the laser beam's electric-field polarization (upper right yellow arrow). (c)-(d) Traces along the nanoparticle parallel (c) and perpendicular (d) to the laser polarization. The color scale shows simulated values of NFE; the dashed lines are AFM height profiles at three different local fluences (from highest to lowest: blue, orange, and green).

shows the direction of electric-field polarization of the laser. As expected, the SU8 is polymerized by NFE along the laser beam polarization. Simulated NFEs are shown along a slice through the nanoparticle parallel to the laser polarization in panel (c), and perpendicular to the laser polarization in panel (d). Also shown as dotted lines are height profiles obtained from AFM at three different locations (i.e. three different local fluences). Qualitatively, the trend in simulated NFE and AFM height contours are similar. However, we observed a lot of variance in the AFM profiles for nanoparticles that should have been irradiated by roughly the same fluence, so our estimations of the NFE (c.f. 109) are quite uncertain. It is not yet clear whether this is due to a nonhomogeneous beam profile or because the NFE is extremely sensitive to small randomization (errors) in the nanoparticle geometry.

The imaged near-field patterns are generally broader than the simulations suggest, which is expected due to limited spatial resolution of AFM (resulting e.g. from tip broadening). Another resolution-limiting factor could be that at high laser fluences, SU8 may be heated, locally, above the glass-transition temperature $T_{g} \approx 55^{\circ} \mathrm{C}[133]$ by thermal conduction from laser-heated gold nanoparticles. Passing the glass transition during the exposure increases the diffusion rate of molecules, thereby potentially 
broadening the polymerized near-field patterns. We note that the increased temperature of SU8 does not actually promote polymerization. Indeed, we observe polymerization caused by plasmonic near fields only near nanoparticle surfaces, where electric charges are expected to accumulate to create large NFEs. Here, the local absorption by the particle is low due to a small charge-current density. Heating of the nanoparticles instead takes place predominantly near the center (here, the center of the "legs") [134], where we observe no nanoparticle-assisted polymerization.

The results could clearly be improved upon by averaging the experimental NFE contours among many nanoparticles irradiated at roughly the same fluence. This is however extremely time consuming. More reliable measurements might be obtained from periodic structures which has been observed to be quite robust against small defects [130]. Our next step is to apply this method on topology-optimized periodic nanostructures designed to resonate at $1500 \mathrm{~nm}$ (coinciding with the Erbium absorption line). This will also help give a better idea of the potential limitations of the method.

\section{Nanoparticle designs}

This section presents a variety of projects combining upconversion materials with photonic structures for light concentration and enhancement of upconversion luminescence. Different host materials $\left(\mathrm{NaYF}_{4}, \mathrm{TiO}_{2}\right)$, photonic designs (topologically optimized structures, back-reflector structures, immersed NCs), and upconverting systems ( $\mathrm{Er}^{3+}$ and organic triplet-triplet-annihilation materials) will be presented, showing the diversity in the frame work developed. All cases share the end goal of achieving the largest possible UCL yield for subsequent absorption in a PV device. The emphasis is on the comparison between numerical calculation and experimental measurements in the first part of the section, while the later parts present the status of ongoing work.

\subsection{Utilizing waveguiding and plasmonic effects}

In a recent study [19], we used DBTO to tailor the design of 3D gold nanostructures placed in a periodic array on top of a $320 \mathrm{~nm}$ thick erbium-doped $\mathrm{TiO}_{2}$ film. A unit cell with period $\Lambda=780 \mathrm{~nm}$ was chosen based on the phase-matching criteria in (80), as this period gives the optimization freedom to trap light in the film either by coupling to a waveguide mode, exciting a plasmon resonance, or a mix of the two [70]. Using the objective function (102) and the max-min formulation in (97) without robustness ( $p=1$ only), the nanostructures were optimized while simultaneously considering excitation of normally incident s- and p-polarized light with wavelengths $\lambda \in\{1490 \mathrm{~nm}, 1500 \mathrm{~nm}, 1510 \mathrm{~nm}\}$. The design domain covers the unit cell in the $x-y$ plane and is set to be $50 \mathrm{~nm}$ high and discretized using $5 \mathrm{~nm}$ wide voxels. The design variables were constrained to be only changing in $2 \mathrm{D}$ and then extruded to $3 \mathrm{D}$, to make designs that can be realized using electron beam lithography. The resulting design can 
Improving the efficiency of upconversion by light concentration using nanoparticle design52

(a)

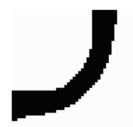

(c)
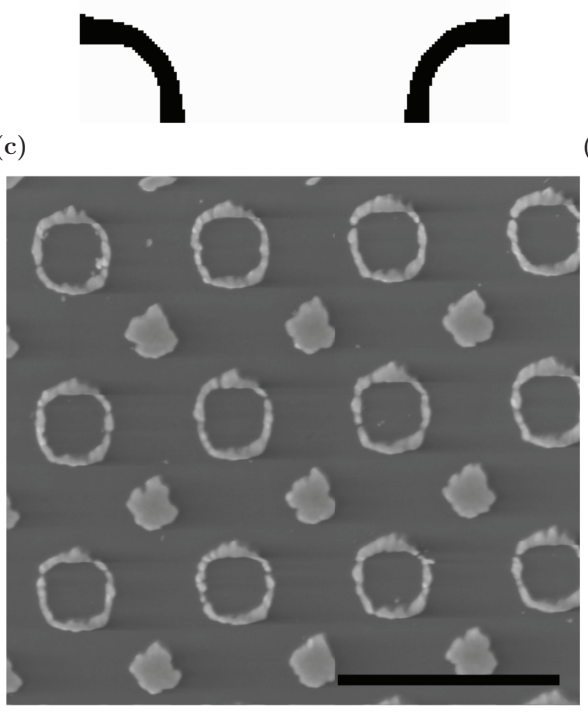

(b)
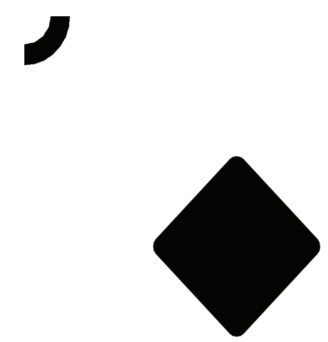

(d)

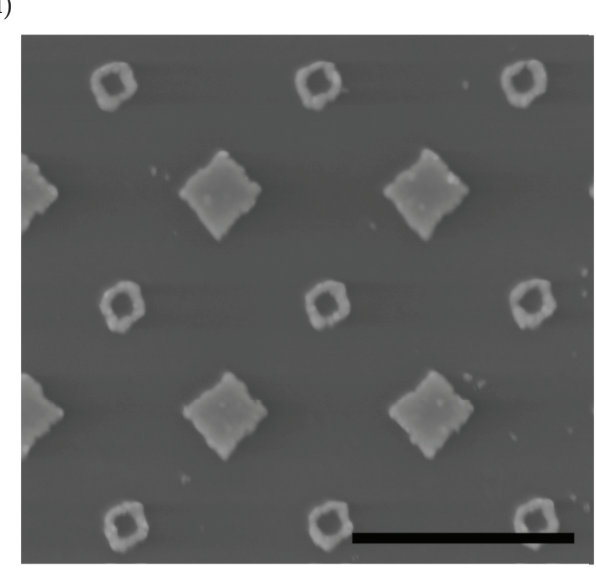

(f)
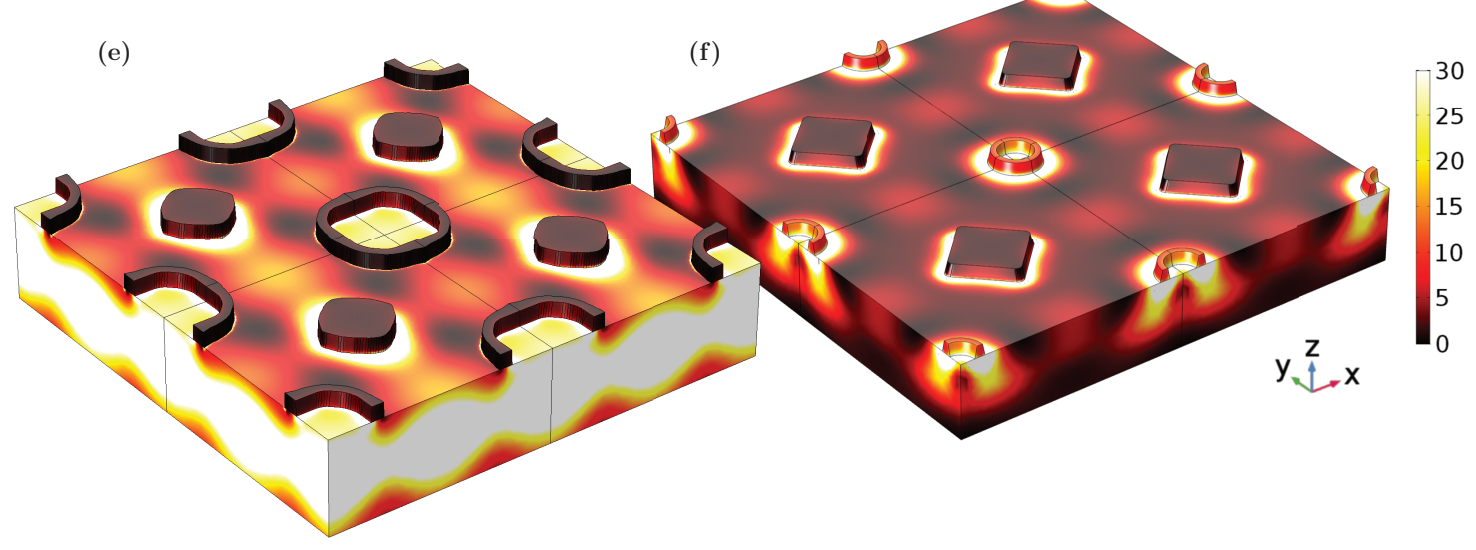

Figure 22. Panels (a) and (b) show unit-cell design of P780* and P1000. Panels (c) and (d) show SEM pictures of EBL-produced arrays, the scalebar is $1 \mu \mathrm{m}$. Panels (e) and (f) show the polarized-average energy-density enhancement $1 / 2 \sum_{\mathrm{s}, \mathrm{p}}|\boldsymbol{E}|^{2} /\left|\boldsymbol{E}_{\mathrm{b}}\right|^{2}$ in the film at $\lambda=1500 \mathrm{~nm}$. A 2-by-2 unit-cell array is shown.

be seen in figure 22(a). The design obtained from the topology optimization appears very simple and can be interpreted as a central square enclosed by four rings. This interpretation forms the basis of a parameterized model used in a second optimization step using three defining parameters; the square width, the ring radius, and ring thickness. The optimization problem was set up to maximize (102) considering again the same wavelengths and polarizations, for a total of three fixed unit-cell periods $\Lambda \in\{780 \mathrm{~nm}, 800 \mathrm{~nm}, 1000 \mathrm{~nm}\}$, in the following denoted by P780, P800 and P1000, 
Improving the efficiency of upconversion by light concentration using nanoparticle design53
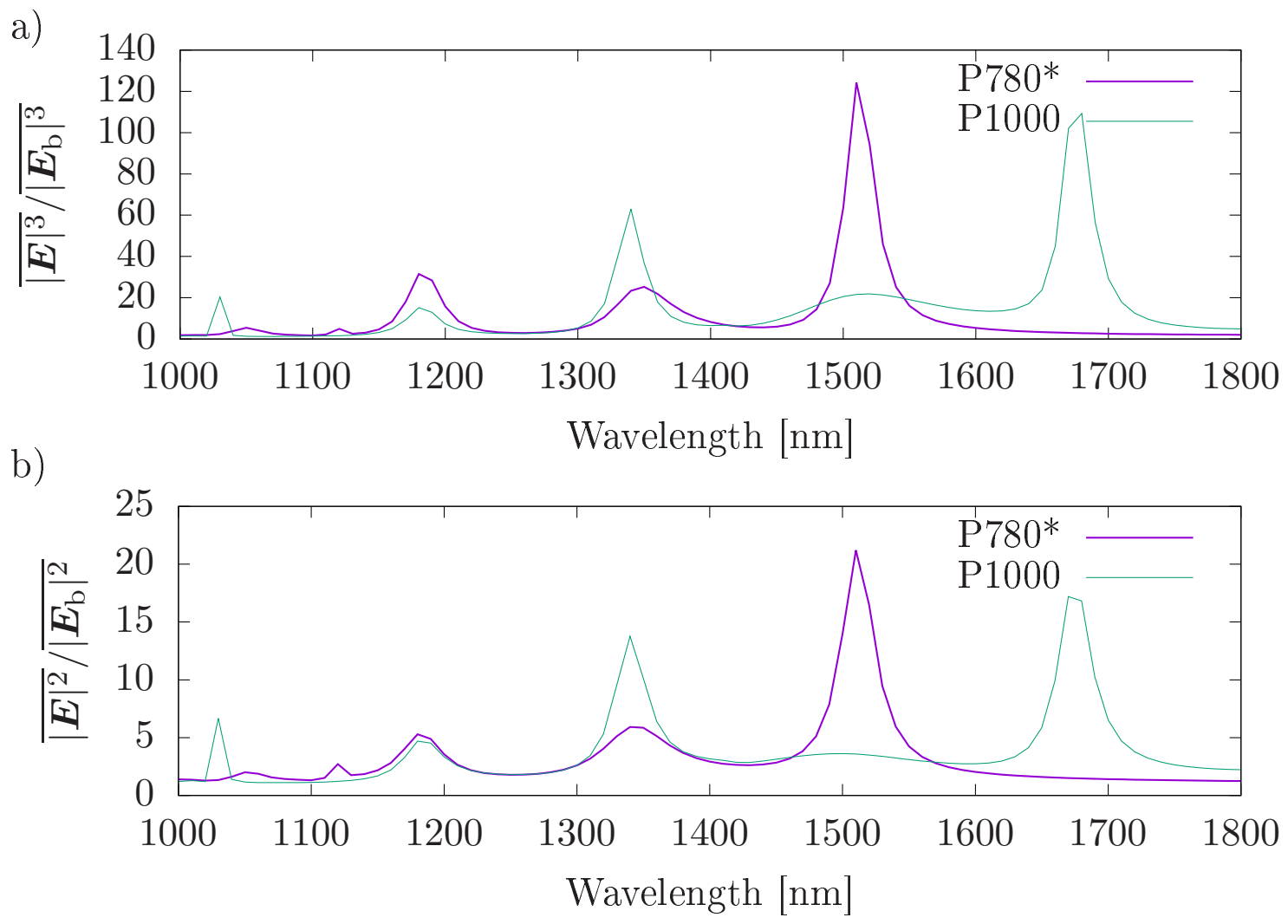

Figure 23. For the two designs, P780* and P1000, panel (a) shows the average $|\boldsymbol{E}|^{3}$ in the presence of nanoparticles normalized to the average $\left|\boldsymbol{E}_{\mathrm{b}}\right|^{3}$ for the background field, corresponding to the important part of the object function in (102). Panel (b) shows the concentration factor $C_{\mathrm{ns}}$ from (32). In all cases, the incoming wave is a plane wave at normal incidence with the electric field along the $x$-direction.

respectively. The original topology-optimized design is denoted by P780*. The result of the optimization with period $1000 \mathrm{~nm}$ can be seen in figure 22(b). Figure 22 shows also SEM pictures of the corresponding EBL-produced arrays and the polarization-averaged energy-density enhancement $1 / 2 \sum_{\mathrm{TM}, \mathrm{TE}}|\boldsymbol{E}|^{2} /\left|\boldsymbol{E}_{\mathrm{b}}\right|^{2}$ in the film at $\lambda=1500 \mathrm{~nm}$. The optimization with varying $\Lambda$ was performed to modify the waveguide coupling efficiency, and one can clearly see how the topology optimized design bears resemblance to that of an excited waveguide mode (figure 22(e)) while the parameter-optimized design shows characteristics of plasmonic hot spots near the gold nanostructures (figure 22(f)) but also traces of the waveguide mode signaling a non-zero, but poor, coupling to the waveguide mode at the $1000 \mathrm{~nm}$ period.

Figure 23(a) shows the approximate UCL-enhancement expression used in the optimization objective function (102) versus the wavelength for the two designs P780* and P1000. Similarly, figure 23(b) shows the concentration factor $C_{\mathrm{ns}}$ from (32). Near a wavelength of $1500 \mathrm{~nm}$, the topology optimized design exhibits a value of the UCL enhancement around 120 while the P1000 design should give around 20 times enhancement. Peaks at other wavelengths are also present and the P1000 design is seen to outperform the P780* design at e.g. $1680 \mathrm{~nm}$ wavelength, where the P1000 design 
couples effectively to a waveguide mode. Not surprisingly, the objective function (102) used to maximize the UCL enhancement is large whenever the concentration factor is large.

The upconversion properties of the samples have been studied experimentally through the intensity dependence of the upconverting film and the UCL enhancements occurring due to the presence of the gold nanostructure. The UCL spectra were obtained by exciting the sample with $1500 \mathrm{~nm}$ laser radiation while recording the resulting luminescence by an integrated spectrometer and CCD detector. First, the intensity dependence of the bare upconverting film was studied by measuring the UCL intensity at various excitation intensities for a fixed angle of incidence and polarization of the excitation source. The UCL yield is computed by integrating the UCL intensity spectra over the $980 \mathrm{~nm}$ peak. The data are fitted to the $f^{(b)}$-function stated in (23) to obtain the saturation intensity described in section 2.1. Hereafter, the UCL enhancement measurements were carried out at varying angles of incidence, for two orthogonal polarizations of the excitation source and for two distinct excitation intensities. To ensure identical collection efficiency for all measurements, the samples were placed in an integrating sphere setup. The measured UCL enhancement for the two designs, P780* and P1000, are shown in figure 24. The solid curves are the theoretically calculated enhancements using (101). Hence, these curves are derived from only the simulated electric fields inside the upconverting film and one experimental parameter - the fitted saturation intensity. Note how the theoretical predictions in figure 24 are able to resolve the order of magnitude of the enhancement at the two widely different excitation intensities, and further, how the qualitative behavior for the different polarizations and angles of incidence are agreeing reasonably. Moreover, the different nature, waveguidelike or plasmonic, of the coupling, discussed in connection with the field plots of figure 22 and the wavelength scans shown in figure 23, is also evident from the UCL enhancement in figure 24. Here, P780* shows large enhancement at normal incidence with large variations for the different angles and polarizations expected for a waveguide resonance while P1000 shows somewhat lower UCL enhancement but with only minor variations with angles and polarizations, features normally attributed plasmonic resonances.

Considering the results for the the P780* structure in figure 24(a,c), a clear increase in the enhancement is observed by lowering the excitation intensity which tells us that even larger enhancements could be obtained by lowering the excitation intensity even further. However, due to the low signal-to-noise ratio of the integrating-sphere setup, it is impractical to keep on lowering the excitation level, and moreover, we would like to shift our attention towards the concentration of the mean-squared electric field inside the upconverting film due to the presence of the nanostructure. This is a more suitable figure of merit for photonic structures, especially when considering the implications the presented upconverters would have on the efficiency of a silicon solar cell. It is not possible to probe this concentration factor directly, but as discussed in section 2.1, it can be indirectly determined through the saturation intensity using (34). In figure 25 the excitation-intensity-dependence data, obtained outside the integrating sphere, is 

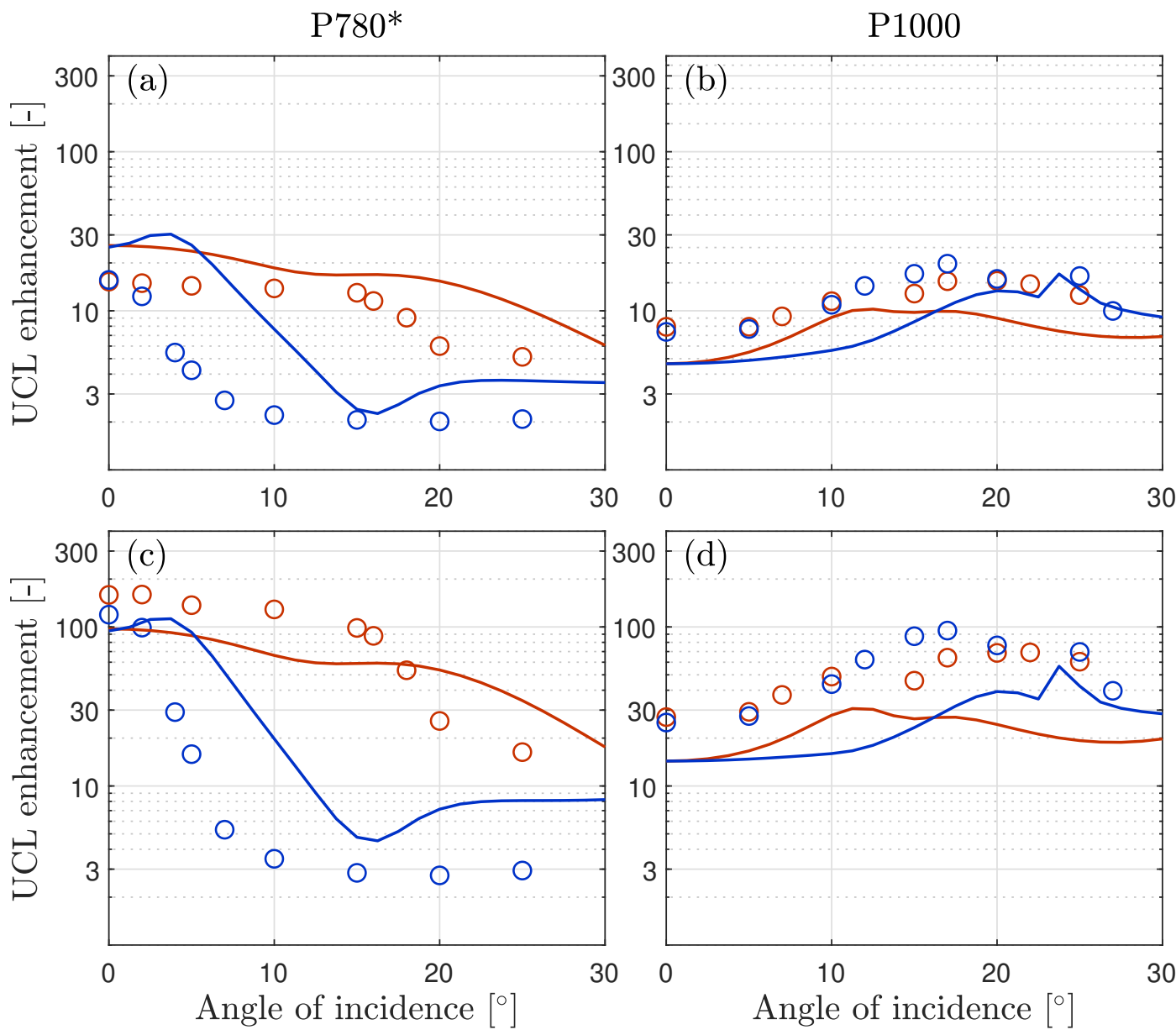

Figure 24. UCL enhancements for the two structures P780* (a,c) and P1000 (b,d) plotted as a function of incidence angle, for both polarization directions with the s-polarized case plotted in blue and the p-polarized case plotted in red. The two excitation intensities are $\mathrm{I}=323 \mathrm{~W} / \mathrm{cm}^{2}(\mathrm{a}, \mathrm{b})$ and $\mathrm{I}=5.8 \mathrm{~W} / \mathrm{cm}^{2}(\mathrm{c}, \mathrm{d})$. The open red or blue circles are measured data whereas the solid curves are theoretically predicted values computed from (101).

plotted both "on" and "off" the structure alongside fitted saturation curves for the P780 sample (this sample is slightly different from P780*, see [19] for further details). The vertical axes have been carefully calibrated to external quantum efficiency as explained in section 2.3. From the fitted saturation intensities, $I_{\mathrm{sat}}^{\mathrm{on}}=(0.59 \pm 0.05) \mathrm{W} / \mathrm{cm}^{2}$ and $I_{\mathrm{sat}}^{\text {off }}=(39 \pm 11) \mathrm{W} / \mathrm{cm}^{2}$, and $\zeta=0.48$ (see [19] for further details), a concentration factor of $C_{\mathrm{ns}}=32 \pm 10$ was determined, consistent with but somewhat large than the simulated value of $\overline{|\boldsymbol{E}|^{2}} / \overline{\left|\boldsymbol{E}_{\mathrm{b}}\right|^{2}}=23$.

In the following we address how the presented concentration factor relates to the quest for a working device for improving the performance of solar cells. Mainly two factors are important to consider here: (I) as discussed in section 2.1 the ideal excitation level for an upconverter for high-internal quantum yield is about an order of magnitude above the saturation intensity, and (II) due to the low absorption of $\mathrm{Er}^{3+}$ 


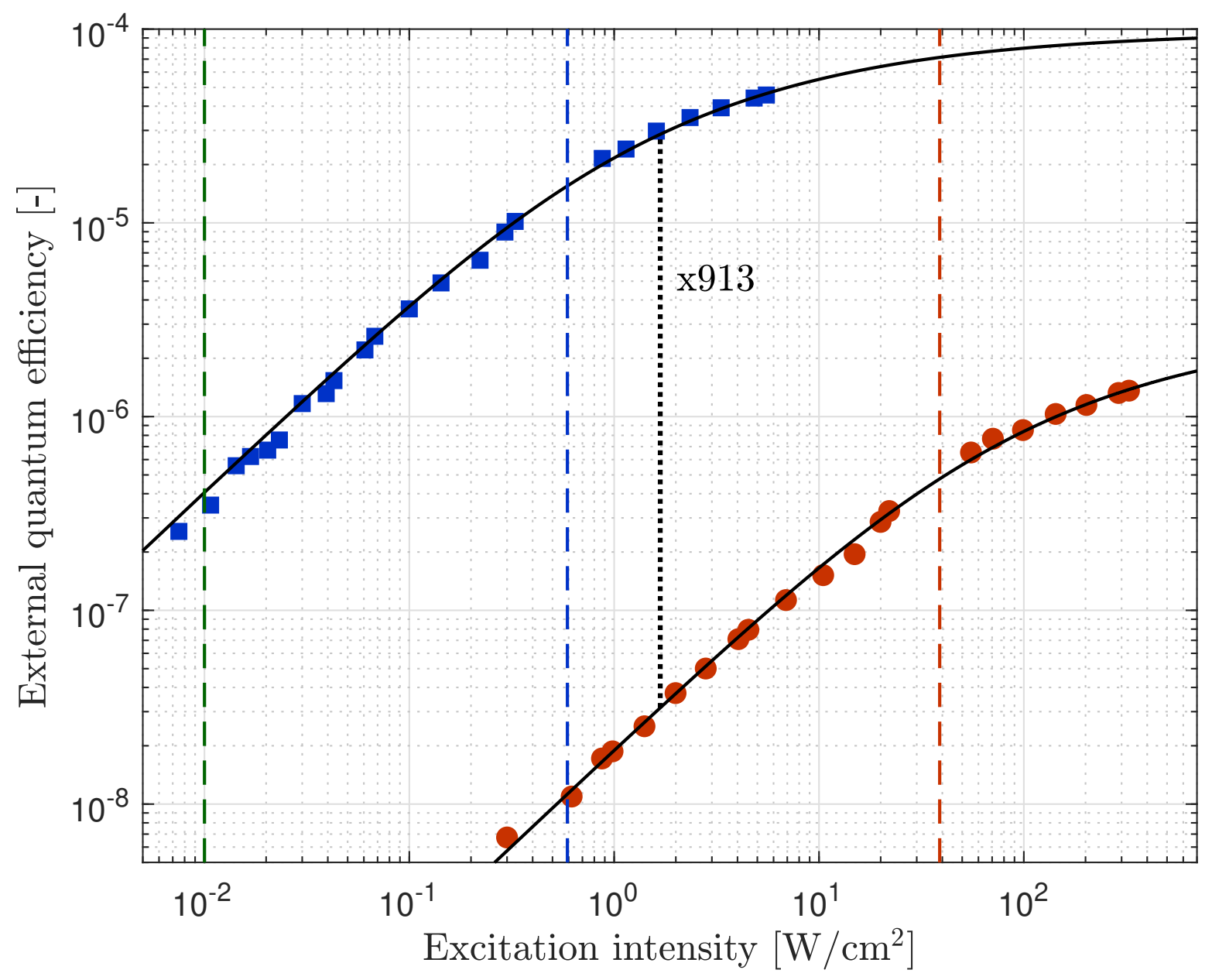

Figure 25. The UCL yield is plotted against excitation intensity for exciting off gold structure (red circles) and on the gold structures (blue squares) with saturation curves (solid black line) found by fitting the data to (23). The vertical scale is re-calibrated to external quantum efficiency as explained in section 2.3 for the "off" structure. The "on" structure is also re-calibrated through a calibrated maximum enhancement factor of 913 (indicated by the black dotted line) measured at $1.7 \mathrm{~W} / \mathrm{cm}^{2}$. Three additional vertical dashed lines are included to show the saturation intensities $I_{\mathrm{sat}}^{\mathrm{off}}(\mathrm{red}), I_{\mathrm{sat}}^{\mathrm{on}}$ (blue), and the maximum non-concentrated intensity available for upconversion, $I_{\mathrm{mnc}}$ (green).

the upconverting film must be much thicker than the films presented in order to absorb a significant amount of the incident light for the current values of the concentration factor. For both (I) and (II), the concentration factor plays an important role. Instead of raising the electromagnetic energy density, the concentration factor can also be viewed as a means for lowering the saturation intensity as stated in (34), and moreover, the total absorption rate (18) in the upconverting film is enhanced by exactly the concentration factor which can thus be seen as a means for lowering the requirements to the thickness of the upconverting film. Considering the upconverting material $\mathrm{TiO}_{2}$ :Er with a saturation intensity of about $40 \mathrm{~W} / \mathrm{cm}^{2}$ (red vertical dashed line in figure 25), another factor of $\approx 600$ larger concentration factor is needed in order to satisfy (I) to get the on- 
structure saturation intensity down to roughly one tenth of $I_{\mathrm{mnc}}$ (i.e. to move the blue vertical dashed line all the way down to one order of magnitude below the green vertical dashed line). That would result in a concentration factor totaling at almost 20,000, which is certainly not feasible. A high concentration factor of a photonic structure will be accompanied by a low frequency bandwidth. The present value of $C_{\mathrm{ns}} \approx 32$ corresponds to a bandwidth (in wavelength) of $\approx 1500 \mathrm{~nm} / 32 \approx 50 \mathrm{~nm}$, which already seems appropriate. Due to (II), the present film structure is also insufficient in the total absorption rate. Assuming the value of $\sigma_{12}$ from (1) to be valid for $\mathrm{TiO}_{2}: \mathrm{Er}$ (which is reasonable due to the agreement between theory and experiment demonstrated in section 2.3), the absorption depth of our $\mathrm{TiO}_{2}$ : $\mathrm{Er}$ films is $\approx 0.6 \mathrm{~mm}$. The light concentration by the nanostructures will effectively reduce this absorption depth by $C_{\mathrm{ns}}$, but our film thickness of $320 \mathrm{~nm}$ is still significantly smaller, resulting in an absorption probability at the percent level. This is one important reason that the predicted external quantum efficiency at $I_{\mathrm{mnc}}$ is only $4 \times 10^{-7}$. We remind that $I_{\mathrm{mnc}}$ in a sense represents 1 sun with the additional assumption of a perfect downshifter which converts the entire solar spectrum in the range $1100 \mathrm{~nm}<\lambda<1450 \mathrm{~nm}$ to the erbium absorption band $1450 \mathrm{~nm}<\lambda<1600 \mathrm{~nm}$ (i.e. converts the yellow band to the red range in figure $1(\mathrm{e})$ and thereby increasing the intensity by roughly a factor of three within the $\mathrm{Er}^{3+}$ absorption band as discussed in the beginning of section 2) without any geometrical concentration. Another important reason for the low external quantum efficiency at $I_{\mathrm{mnc}}$ is the strong contribution of non-radiative decay channels in $\mathrm{TiO}_{2}$ : $\mathrm{Er}$ as discussed in section 2.3. With that said, $\mathrm{TiO}_{2}$ has been a very strong work horse in our research due to the high level of control and reproducibility in the synthesis process. Furthermore, with a high refractive index $n_{\mathrm{R}} \approx 2.2$, this material has demonstrated that waveguide effects present a very feasible method for achieving a large light concentration factor and in turn a large enhancement factor of the UCL yield.

Turning our speculations towards the better host material $\mathrm{NaYF}_{4}$ with a saturation intensity of $0.5 \mathrm{~W} / \mathrm{cm}^{2}$, see figure 7 , we would still need an order of magnitude larger concentration factor than found here to satisfy (I), which would be challenging since this material has a lower refractive index making efficient waveguide coupling difficult. Moreover, as explained above, further concentration comes at the expense of lowering the bandwidth of the photonic structure even further, which is not feasible. However, the extra concentration factor could be achieved through a geometrical concentration of light in the downshifter material, which would increase the available intensity reaching the upconverter material beyond the $I_{\mathrm{mnc}}$. This is known as fluorescent concentration and is well-described in $[1,2]$. One should then just bear in mind due to (II) that the concentration factor $C_{\mathrm{ns}} \approx 32$ effectively reduces the absorption depth of $0.8 \mathrm{~mm}$ (for $\mathrm{NaYF}_{4}: \mathrm{Er}$ ) to about $25 \mu \mathrm{m}$, which is thus the ball-park estimate of the film thickness in which this concentration factor $C_{\mathrm{ns}}$ must be achieved. It should also be noted that, at least for our synthesized $\mathrm{NaYF}_{4}$ :Er nanoparticles, the emission quantum efficiency $A_{31} / \Gamma_{31} \approx 10 \%$ must also be improved. All these considerations taken together, it seems that the most ideal way forward would be to find new upconverting materials 
with stronger absorption and at the same time a large refractive index. This would significantly lower the demands set by (I) and (II) while the large refractive index ensures the possibility of efficient waveguide coupling and thus allowing for the large concentration of the electromagnetic energy density as demonstrated here.

\subsection{Au-nanostructures with $E r^{3+}$-doped $\mathrm{NaYF}_{4} \mathrm{NCs}$}

The truncated cone shape (i.e. nanodisc) is the possibly simplest geometry that can be fabricated with a short EBL processing time. Therefore, we fabricated Au-nanodiscs both on top of $\mathrm{NaYF}_{4}$ :Er NC monolayers and buried underneath the monolayers after being deposited on quartz. The designing of a simple geometry of Au-structures with the thinnest possible film of the NCs is the best way to reveal the physics behind the interaction between the Au-nanodiscs and the emitters (i.e. the $\operatorname{Er}^{3+}$ ions). The fabrication of Au-nanodiscs on top of a single layer of NCs was an intriguing task. The design of the buried-Au configuration, on the other hand, involved a simple spin-coating of a pre-defined concentrated NCs solution on a Au-disc patterned quartz substrate. The experimental details of both fabrications are mentioned in [23]. Experimentally, the Au-nanodiscs were fabricated in $1 \times 1 \mathrm{~mm}^{2}$ regions such that measurements on and off Au-nanodiscs could be performed on a single sample. This reduces the systematic effects of variation in sample fabrication. The localized surface plasmon resonance peak position of the nanodisc was matched with the excitation wavelength of $1500 \mathrm{~nm}$ by altering the diameter of the discs while keeping the height and shape of structures constant. The scattering and absorption cross sections of the nanodiscs were calculated using the methods described in section 3, and the sum of both corresponds to the theoretical extinction cross section. Dividing this with the disc area, neutralized the size variation effect, leading to the relative extinction cross section, $\sigma_{E} / A$. Experimentally, we determined the extinction cross section by measuring absorption and scattering of light by the nanodiscs. A comparison between both is presented in figure 26 for Au-discs buried monolayers configuration. The figure shows a nice match in the peak positioning of the experimentally designed Au-discs guided with the simulated parameters.

The fabrication of Au-discs on top of the monolayers was successfully implemented. It was, however, found that the upconversion of the monolayers drastically dropped after the EBL process. This could possibly be due to defect formation in the NCs by the e-beam. Despite the successful proof of concept, we thus chose to proceed all the experimental advances with the Au-buried monolayers configurations. We refer to [23] for an elaborate discussion of these efforts.

Fabrication of topologically optimized structures are in progress with the micron sized thick $\mathrm{Er}^{3+}$-doped $\mathrm{NaYF}_{4} \mathrm{NC}$ films. Previously, in section 2.2, we discussed the fabrication of such thick films via spin-coating and we are currently fabricating such films on a quartz substrate coated with topologically optimized Au structures. In this way the samples will be in the buried-structures configuration. Currently, we are in the process of designing the samples and further comparison between the numerical 

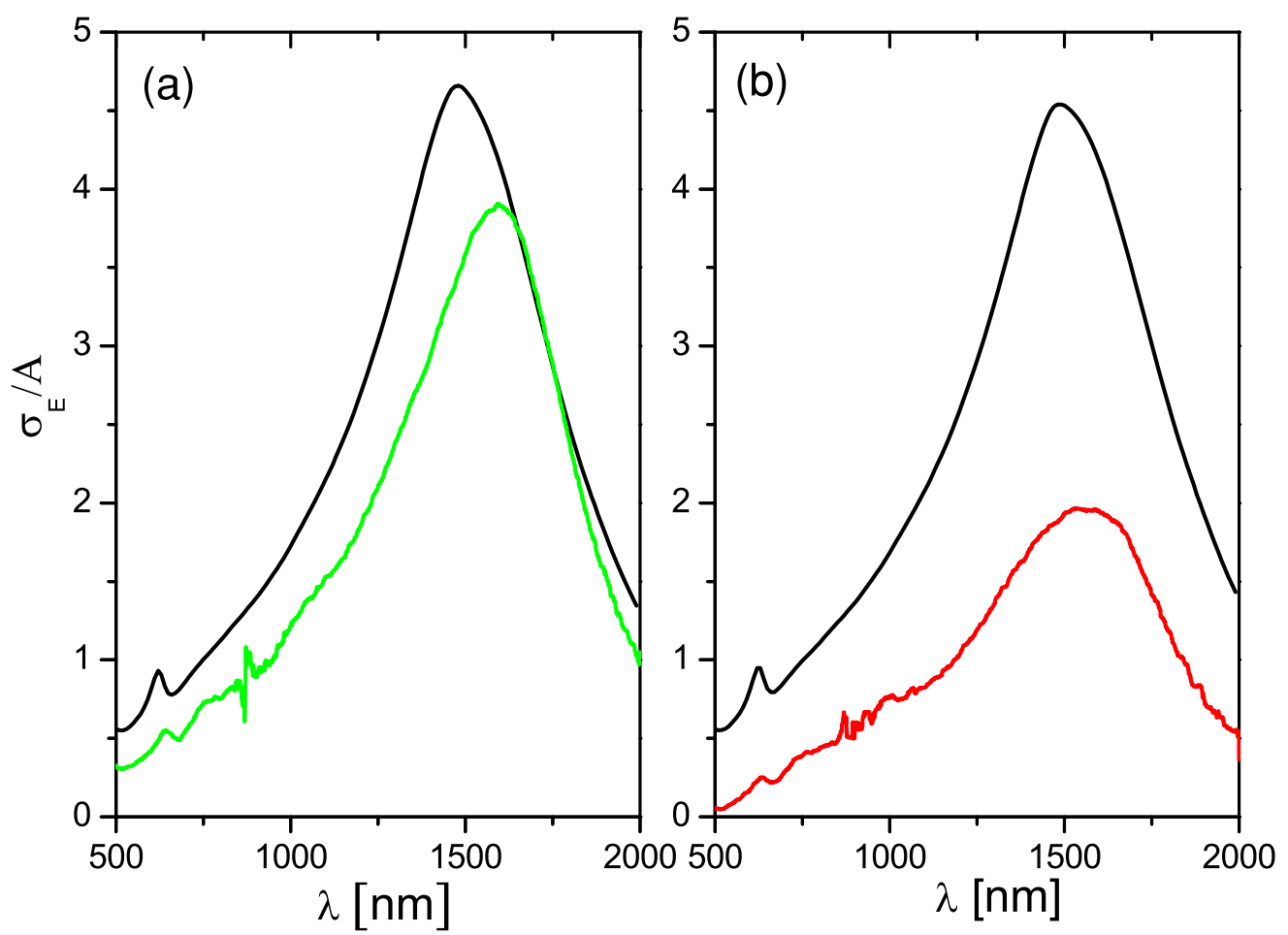

Figure 26. The experimental (colored curves) and calculated (black curves) relative extinction cross section showing the localized surface plasmon resonance peak positioning of Au-discs buried in the monolayers of core (a) and core with thickest shell (b) NCs.

calculations and the experimental results will be carried out.

\subsection{A back-reflector-based design}

Another investigated approach for attaining localized field enhancement in the erbiumdoped $\mathrm{TiO}_{2}$ film is to introduce a planar reflector/mirror (a back reflector) below this active region and an array of optimized Au-nanoparticles above it. The back-reflector ensures that no light propagates past the $\mathrm{TiO}_{2}$ film and may serve to reflect upconverted light back into the relevant layer of the solar cell. One obvious limitation of this approach is that only a single layer of $\mathrm{TiO}_{2}$ film including a back-reflector can be included. Nonetheless, the approach has been found to yield promising results as will be demonstrated with the following numerical example.

This example consider a $\Lambda=800 \mathrm{~nm}$ (x,y)-periodic unit-cell, with mirror symmetry imposed along both the $\mathrm{x}$ - and $\mathrm{y}$-direction. The model geometry consists of a substrate of $\mathrm{Si}$ on which a $100 \mathrm{~nm}$ thick layer of Au, acting as the back-reflector, is placed. A 320 $\mathrm{nm}$ thick TiO2 film is situated on top of the Au layer and an array of $100 \mathrm{~nm}$ high Auparticles are placed on top of the TiO2 film. The full setup is placed in an air background. 
(a)

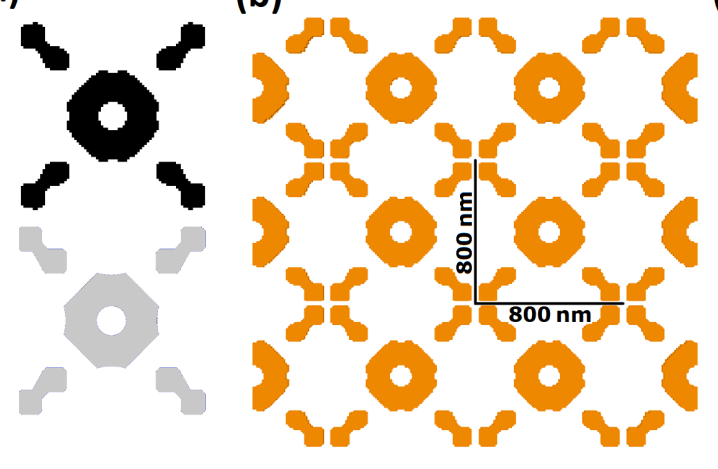

(c)

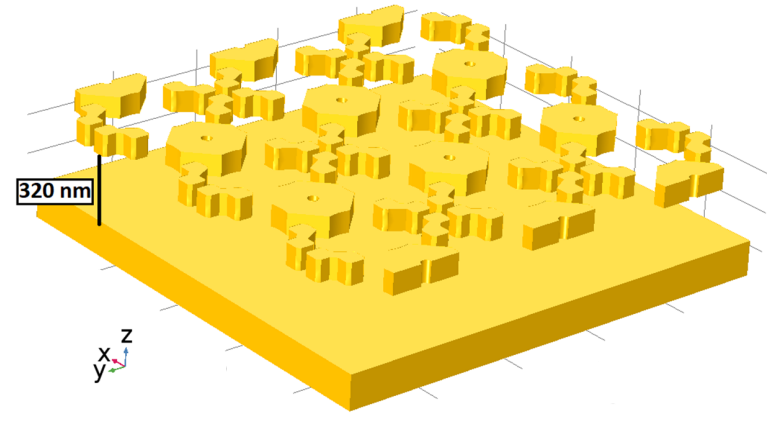

Figure 27. The top of panel (a) show a top-down view of final design from DBTO procedure and the bottom show a parameterized version of the final design. Panel (b) show a top-down view of 3 by 3 unit cells of the optimized Au-particle geometry. Panel (c) show a side view of the final parametrized Au-particle geometry and Au-back reflector surrounding the $\mathrm{TiO} 2$ region (not shown).

The Au-particles are designed to operate in the wavelength band $\lambda \in[1480 \mathrm{~nm}, 1560 \mathrm{~nm}]$ for both s- and p-polarizations at normal incidence.

The Au-particle geometries and placements are designed using topology optimization as described in section 4 . The physics is modeled in the frequency domain using (45) and solved using the finite element method with first order Nedelec elements [49]. The design problem is solved as a max/min-problem, (97)-(99), simultaneously considering normal incident s- and p-polarized light for the three wavelengths $\lambda \in$ $\{1480 \mathrm{~nm}, 1520 \mathrm{~nm}, 1560 \mathrm{~nm}\}$. The design domain is discretized using $10 \mathrm{~nm}$ voxels and the objective function (102) is evaluated $20 \mathrm{~nm}$ from all Au-surfaces to avoid the function benefiting from field-enhancement in regions where upconversion quenched.

A top-down view of the final design field and a post processed version are shown in figure 27(a). An array of 3 by 3 unit cells of the final particle design cross section is presented in figure 27(b) and a side view of the final Au-distribution for a simple parametrization of the final particle design is shown in figure 27(c).

The final design in figure 27(a) [top] is post processed to remove the $10 \mathrm{~nm}$ staircase features stemming from the discretization. This post processing has the effect of shifting the height at which the best performance is obtained from $100 \mathrm{~nm}$ to $\approx 110 \mathrm{~nm}$. The spatially averaged enhancement of $|\boldsymbol{E}|^{3}$ in the $\mathrm{TiO}_{2}$-film (evaluated $20 \mathrm{~nm}$ from all $\mathrm{Au}$ surfaces), attained by introducing the Au nanoparticles and back reflector (the objective function value), for the $110 \mathrm{~nm}$ high particle array, is presented in figure 28(a).

From the graph it is seen that an average enhancement of $>90$ is obtained across the full wavelength interval of interest with the per frequency enhancement remaining above 50 and peaking at 135 at $\lambda=1530 \mathrm{~nm}$. It is also observed that the DBTO procedure has clearly optimized the Au-particle to exhibit high performance in the desired wavelength region, as the performance is seen to deteriorate rapidly when leaving the region. Further, it appears as if two resonance peaks exists in the targeted interval 

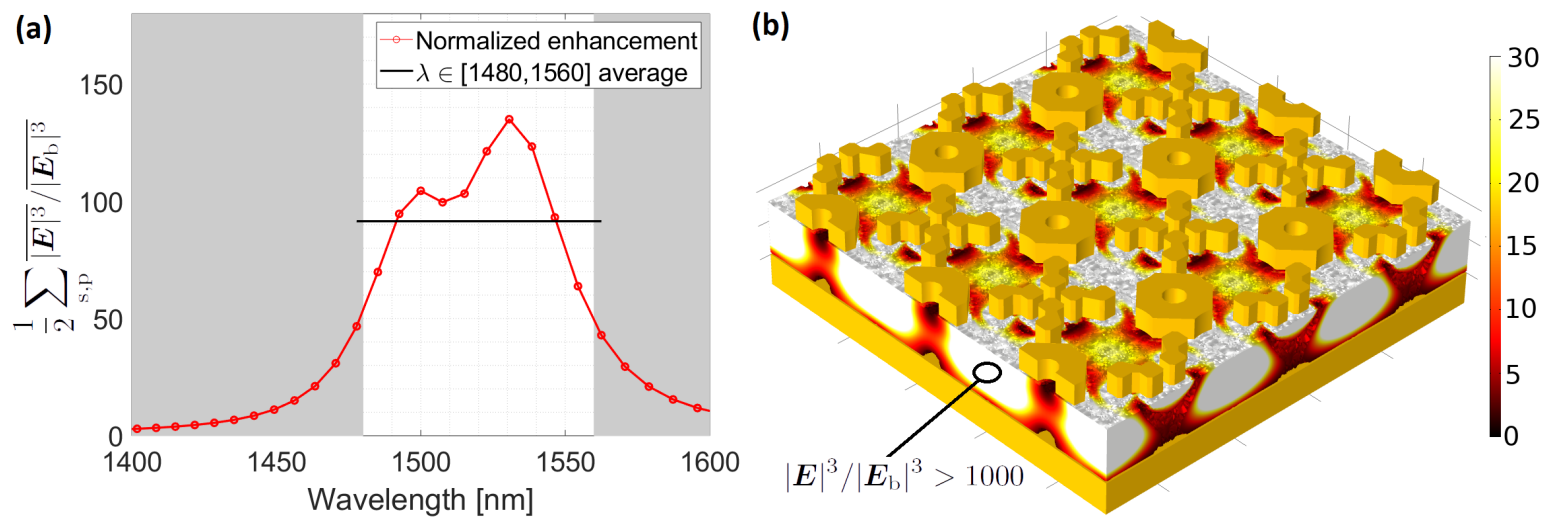

Figure 28. Panel (a) show the spatially averaged enhancement of $|E|^{3}$ in the $\mathrm{TiO}_{2}$-film (evaluated $20 \mathrm{~nm}$ from all Au-surfaces), attained by introducing the Au nanoparticles and back reflector (the objective function value), for the $110 \mathrm{~nm}$ high particle array in figure 27. Panel (b) show the polarization averaged energy-density enhancement at $1500 \mathrm{~nm}$ in the $\mathrm{TiO}_{2}$ film.

centered approximately at $\lambda=1500 \mathrm{~nm}$ and $\lambda=1530 \mathrm{~nm}$. This observation is supported when looking at the $|\boldsymbol{E}|^{3}$ distribution in the $\mathrm{TiO}_{2}$-film.

The simulated polarization averaged energy-density enhancement at $1500 \mathrm{~nm}$ is shown in figure 28(b). Firstly, it is clear from the figure that a large energy-density enhancement occurs near the Au-particles, which is likely attributable to localized plasmonic enhancement. Secondly, a large enhancement is observed in localized regions between the Au back-reflector and the An-particles, which may be described as localized cavity resonances in the $\mathrm{TiO}_{2}$ film. Thirdly, a non-local waveguiding effect also contributes to the overall energy-density enhancement in the structure. It is noted that spatially localized enhancements of $|\boldsymbol{E}|^{3}$ above a 1000 are observed in the pockets in the middle of the $\mathrm{TiO}_{2}$-film, more than $100 \mathrm{~nm}$ from any Au-surfaces, at $\lambda=1500$ nm.

This example clearly demonstrates the usefulness DBTO as a design tool for achieving significant wideband enhancements of the electric field, for a setup consisting of a layer of Au-nanoparticle on top of an active region with a Au back-reflector underneath.

\subsection{Upconversion in organic photovoltaics (OPV): Optimized organic solar cell design}

UC layers can be integrated in OPV using multiple different strategies, which overall can be separated into integration external or internal to the OPV stack. These integration schemes are demonstrated in Figure 29. Figure 29a shows an example of an upconversion layer integrated outside a semi-transparent OPV stack, which we have recently pursued. In Figure 29b, a representative J-V curve of such a cell is shown. In this case, the cell is developed from the active layer material system $\mathrm{PCE} 11: \mathrm{PC}_{70} \mathrm{BM}$, where $\mathrm{ZnO}$ was used as ETL and PEDOT:PSS used as HTL. The organic solar cell reached a device efficiency of up to $6.5 \%$, showing a small additional photocurrent arising from the UC 
layer. Few other reports have been made for external UC layers integration in OPV cells $[135,136,137]$. The group of Brabec demonstrated that $\mathrm{NaYF}_{4}: \mathrm{Yb} / \mathrm{Er}$ hexagonal nanoplates can be integrated as UC layers outside an OPV cell, when embedded in PMMA matrices [137]. The cells provided a short-circuit current density $\left(\mathrm{J}_{\mathrm{sc}}\right)$ of $450 \mu \mathrm{A}$ $\mathrm{cm}^{-2}$ under $975 \mathrm{~nm}$ laser diode illumination (at $200 \mathrm{~mW} / \mathrm{cm}^{2}$ ), leading to around $0.1 \%$ pure UC PCE of the cell. The total PCE of the cell upon 1 sun illumination was $2.5 \%$ for these P3HT:PC ${ }_{61} \mathrm{BM}$ based UC OPV devices, and reached a $1.6 \% \mathrm{~J}_{\mathrm{sc}}$ enhancement at one sun illumination. Adikaari et al. used $\mathrm{Yb}^{3+}: \mathrm{Ho}^{3+}$ co-doped $\mathrm{Y}_{2} \mathrm{BaZnO}_{5}$ near-infrared up-converting phosphors, and demonstrated a short-circuit current $\left(\mathrm{J}_{\mathrm{sc}}\right)$ of $16 \mu \mathrm{A} \mathrm{cm}^{-2}$ under $986 \mathrm{~nm}$ laser diode illumination (at $390 \mathrm{~mW} / \mathrm{cm} 2$ ) in PCDTBT:PCBM OPV cells [136]. Schulze et al. demonstrated an enhancement in $\mathrm{J}_{\mathrm{sc}}$ of $0.16 \cdot 10^{-3} \mathrm{~mA} / \mathrm{cm}^{2} / \odot^{2}$ for P3HT:ICBA OPV devices using the nitroaminopalladiumtetrakis porphyrin/rubrene molecular pair as the upconverter [135]. The increase in short circuit current was reported per suns squared, which makes the value a figure-of-merit measuring the increase at the illumination density equal to 1 sun. The decoupled device and UC layer optimization is a clear advantage for UC layers integrated outside the cells, although a semitransparent OPV cell is required, which puts extra constraints on the top electrode. In such UC OPV device configurations, a back-reflector is used to efficiently couple the visible UC light back into the cell. Alternatively, the UC layer is integrated internally in the OPV cell, which brings along the advantage of fewer fabrication steps and potentially less photon losses. The Brabec group demonstrated a UC photocurrent from $\mathrm{MoO}_{3} \mathrm{Yb}^{3+} / \mathrm{Er}^{3+}$ hole transport layers contributing to the slightly enhanced $\mathrm{J}_{\mathrm{sc}}$ of such UC OPV devices (although less than 1\% under one sun illumination), showing a proofof-concept method for internal UC integration in OPV [138]. However, in this method, the interlayer should be optimized for both upconversion and hole transport, which may lead to non-ideal performance properties. As a positive addition, many of the UC layers are already embedded inside a metal oxide matrix, in our case e.g. $\mathrm{TiO}_{x}$ deposited by sputtering. Sputtered titanium and molybdenum oxide interlayers have recently been introduced as efficient and stable transport layers in OPV cells [139, 140], and may be utilized for such UC design schemes. A joint consideration for internal and external integration is the position of $\mathrm{UC}$ layer with respect to the cell. Absorption/reflection losses in the visible regime are clear challenges for front-side integration, but it comes along with higher degree of fabrication freedom when internal cell integration is pursued. For external cell integration, this makes back-side integration the preferred choice [136], although semitransparent cells are here required.

Plasmonically enhanced upconversion is still to be demonstrated for OPV cells. As for cell design, plasmonically enhanced upconversion would follow similar routes and guidelines as those set out for pure UC layers. It should be noted that integration of nanoparticles inside an interlayer, i.e. in order to enhance photon upconversion in that layer, may affect not only the optical properties, but also the electrical properties of the solar cell, if the structures are partially transferred to layer surface. In fact, such nanostructured interfaces may in OPV cells contribute with a positive electrical effect, 
a)

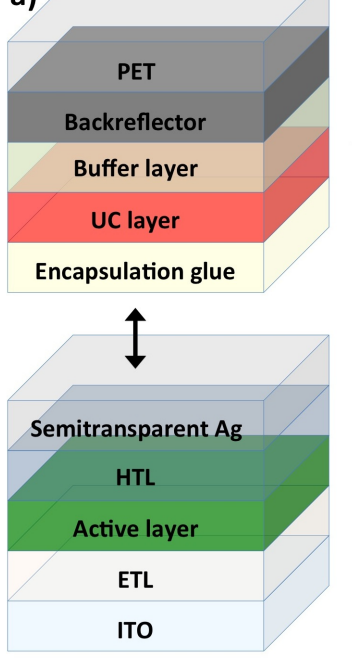

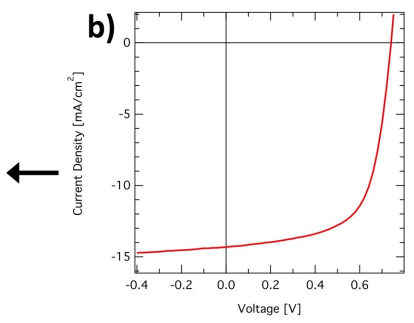

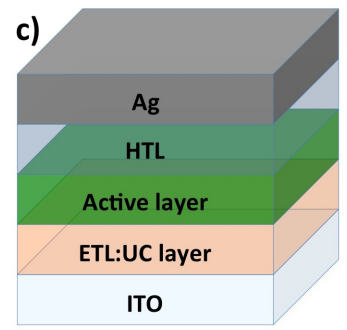

Figure 29. Panel (a) show an example of UC layer integration outside an organic solar cell stack. Panel (b) show a representative J-V curve of the cell design shown in (a), using PCE11:PC $70 \mathrm{BM}$ as the active layer, and $\mathrm{ZnO}$ and PEDOT:PSS as ETL and HTL, respectively. Panel (c) show an example of UC layer integrated inside the ETL of an organic solar cell.
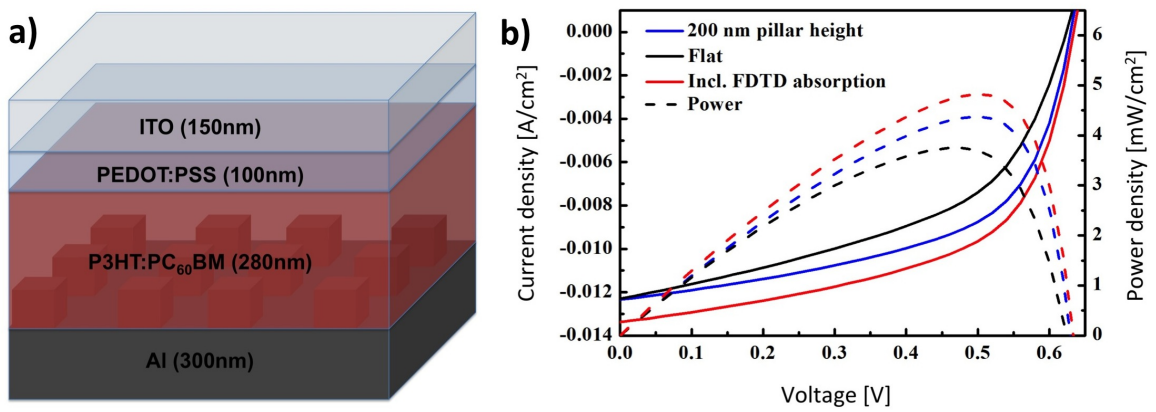

Figure 30. Panel (a) shows the design of the nanostructured OPV cell studied in this work. Panel (b) shows the electrical properties of reference OPV (black), a nanostructured OPV with the optical generation profile equalized to the planar cell (blue) and a nanostructured OPV with an optical generation profile calculated from FDTD (red). The total PCE improvement, incl. optical and electrical effects are $28.5 \%$ [141]. Figure adapted from reference [141].

in addition to the desired optical effect. In recent work [141], we have demonstrated that OPV cells containing squared nanostructures at the active layer and interlayer interface, can lead to a $18 \%$ fill factor enhancement as compared to the planar OPV cells, due to an improved charge collection efficiency in the nanostructured organic solar cells (Figure $30)$. 


\section{Conclusion}

Upconversion of sunlight in order to better utilize the solar spectrum in photovoltaics is a promising method for increasing the short-circuit current, but the technique still has its challenges. The main challenge is the rather poor absorption of the basic materials, which severely limits the total upconversion efficiency. Much of the poor absorption can be counter-acted by utilizing downshifting to effectively increase the available light in the absorption band, and by the use of modern optical design techniques, such as topological optimization, to design devices capable of delivering high field concentration. There is, however, still some way to a commercially viable device. Based on the discussions in this review, it is evident that decreasing the value of $I_{\text {sat }}$ is paramount, and it will likely also be necessary to further effectively decrease it by utilizing efficient nanoscale electric field concentrators. As demonstrated, most of the setup for doing this is well-known, but the methods for field enhancement presented generally work best for materials with a higher refractive index. Future research in the field can therefore be focused on the search for new materials with lower values of $I_{\text {sat }}$ and higher refractive indices.

\section{Acknowledgments}

We acknowledge the support from Innovation Fund Denmark through the SunTune project, Aarhus University Centre for Integrated Materials Research (iMAT), the NATEC Center of Excellence (Grant No. 8692) granted by VILLUM FONDEN and research grant (17677) from VILLUM FONDEN.

\section{Bibliography}

[1] Goldschmidt J C, Loper P, Fischer S, Janz S, Peters M, Glunz S W, Willeke G, Lifshitz E, Kramer K and Biner D 2008 Advanced upconverter systems with spectral and geometric concentration for high upconversion efficiencies 2008 Conference on Optoelectronic and Microelectronic Materials and Devices (IEEE) URL https://doi.org/10.1109/commad.2008.4802153

[2] Goldschmidt J C 2009 Novel solar cell concepts Ph.D. thesis University of Konstanz

[3] Moraitis P, Schropp R and van Sark W 2018 Optical Materials 84 636-645 URL https://doi.org/10.1016/j.optmat.2018.07.034

[4] Moreels I, Lambert K, Smeets D, Muynck D D, Nollet T, Martins J C, Vanhaecke F, Vantomme A, Delerue C, Allan G and Hens Z 2009 ACS Nano 3 3023-3030 URL https://doi.org/10.1021/nn900863a

[5] Takeda Y, Mizuno S, Luitel H N and Tani T 2016 Appl. Phys. Lett. 108043901 URL https://doi.org/10.1063/1.4940442

[6] Takeda Y, Mizuno S, Luitel H N and Tani T 2018 J. Am. Ceram. Soc. 102 3457-3467 URL https://doi.org/10.1111/jace.16193

[7] Polman A and Atwater H A 2012 Nature Materials 11 174-177 URL https://doi.org/10.1038/nmat3263

[8] Huang X, Han S, Huang W and Liu X 2013 Chem. Soc. Rev. 42 173-201 URL https://doi.org/10.1039/c2cs35288e

[9] Strümpel C, McCann M, Beaucarne G, Arkhipov V, Slaoui A, Švrček V, del 
Improving the efficiency of upconversion by light concentration using nanoparticle design65

Cañizo C and Tobias I 2007 Sol. Energy Mater. Sol. Cells 91 238-249 URL https://doi.org/10.1016/j.solmat.2006.09.003

[10] Shang Y, Hao S, Yang C and Chen G 2015 Nanomaterials 5 1782-1809 URL https://doi.org/10.3390/nano5041782

[11] van Sark W G, de Wild J, Rath J K, Meijerink A and Schropp R E 2013 Nanoscale Res. Lett. 8 URL https://doi.org/10.1186/1556-276x-8-81

[12] Ramasamy P, Manivasakan $\mathrm{P}$ and Kim J $2014 \quad R S C$ Adv. 4 34873-34895 URL https://doi.org/10.1039/c4ra03919j

[13] Gueymard C, Myers D and Emery K 2002 Solar Energy 73443 - 467 ISSN 0038-092X URL http://www.sciencedirect.com/science/article/pii/S0038092X03000057

[14] Fischer S, Martín-Rodríguez R, Fröhlich B, Krämer K W, Meijerink A and Goldschmidt J $\quad$ C 2014 Journal of Luminescence $\mathbf{1 5 3} 281-287$ URL https://doi.org/10.1016/j.jlumin.2014.03.047

[15] Milonni P W and Eberly J H 2010 Laser Physics (John Wiley \& Sons, Inc.) URL https://doi.org/10.1002/9780470409718

[16] Fischer S 2014 Upconversion of Sub-Band-Gap Photons for Silicon Solar Cells Ph.D. thesis University of Freiburg

[17] Fischer S, Steinkemper H, Löper P, Hermle M and Goldschmidt J C 2012 Journal of Applied Physics 111013109 URL https://doi.org/10.1063/1.3674319

[18] Christiansen J, Lakhotiya H, Eriksen E, Madsen S P, Balling P and Julsgaard B 2019 Journal of Applied Physics 125043106 URL https://doi.org/10.1063/1.5064409

[19] Christiansen J, Vester-Petersen J, Roesgaard S, Møller S H, Christiansen R E, Sigmund O, Madsen S P, Balling P and Julsgaard B 2019 Submitted

[20] Bahtat A, Bouazaoui M, Bahtat M, Garapon C, Jacquier B and Mugnier J 1996 Journal of Non-Crystalline Solids 202 16-22 URL https://doi.org/10.1016/0022-3093(96)00172-x

[21] Lakhotiya H, Christiansen J, Hansen J L, Balling P and Julsgaard B 2018 Journal of Applied Physics 124 163105 URL https://doi.org/10.1063/1.5037059

[22] Johannsen S R, Roesgaard S, Julsgaard B, Ferreira R A S, Chevallier J, Balling P, Ram S K and Larsen A N 2016 Opt. Mater. Express 6 1664-1678 URL http://www . osapublishing. org/ome/abstract. cfm?URI=ome-6-5-1664

[23] Lakhotiya H, Nazir A, Roesgaard S, Eriksen E, Christiansen J, Bondesgaard M, van Veggel F C J M, Iversen B B, Balling P and Julsgaard B 2019 ACS Appl. Mater. Interfaces 11 1209-1218 URL https://doi.org/10.1021/acsami.8b15564

[24] Lakhotiya H, Nazir A, Madsen S P, Christiansen J, Eriksen E, Vester-Petersen J, Johannsen S R, Jeppesen B R, Balling P, Larsen A N and Julsgaard B 2016 Applied Physics Letters 109263102 URL https://doi.org/10.1063/1.4972785

[25] Balling P, Christiansen J, Christiansen R, Eriksen E, Lakhotiya H, Mirsafaei M, Møller S, Nazir A, Vester-Petersen J, Jeppesen B, Jensen P, Hansen J, Ram S, Sigmund O, Madsen M, Madsen S and Julsgaard B 2018 Opt. Mater. 83 279-289 URL https://doi.org/10.1016/j.optmat.2018.06.038

[26] Fischer S private communication

[27] Pedrini J and Monguzzi A 2017 J. Photon. Energy 8022005 URL https://doi.org/10.1117/1.JPE.8.022005

[28] Hoseinkhani S, Tubino R, Meinardi F and Monguzzi A 2015 Phys. Chem. Chem. Phys. 174020 4024 ISSN 1463-9076 URL http://dx.doi.org/10.1039/C4CP03936J

[29] Monguzzi A, Oertel A, Braga D, Riedinger A, Kim D K, Knüsel P N, Bianchi A, Mauri M, Simonutti R, Norris D J and Meinardi F 2017 ACS Appl. Mater. Interfaces 9 40180-40186 ISSN 1944-8244 URL https://doi.org/10.1021/acsami.7b10829

[30] Lissau J S, Gardner J M and Morandeira A 2011 J. Phys. Chem. C 115 23226-23232 ISSN 1932-7447 URL http://dx.doi.org/10.1021/jp209774p

[31] Ekins-Daukes N J and Schmidt T W 2008 Appl. Phys. Lett. 93063507 
Improving the efficiency of upconversion by light concentration using nanoparticle design66

[32] Lissau J S, Nauroozi D, Santoni M P, Ott S, Gardner J M and Morandeira A 2015 J. Phys. Chem. C 119 25792-25806 ISSN 1932-7447 URL http://dx.doi.org/10.1021/acs.jpcc.5b08907

[33] Lissau J S, Nauroozi D, Santoni M P, Ott S, Gardner J M and Morandeira A 2013 J. Phys. Chem. C 117 14493-14501 ISSN 1932-7447 URL http://dx.doi.org/10.1021/jp402477q

[34] Lissau J S, Nauroozi D, Santoni M P, Edvinsson T, Ott S, Gardner J M and Morandeira A 2015 J. Phys. Chem. C 119 4550-4564 ISSN 1932-7447 URL http://dx.doi.org/10.1021/jp5118129

[35] Simpson C, Clarke T M, MacQueen R W, Cheng Y Y, Trevitt A J, Mozer A J, Wagner P, Schmidt T W and Nattestad A 2015 Phys. Chem. Chem. Phys. 17 24826-24830 ISSN 1463-9076 URL http://dx.doi.org/10.1039/C5CP04825G

[36] Hill S P, Dilbeck T, Baduell E and Hanson K 2016 ACS Energy Lett. 1 3-8 URL http://dx.doi.org/10.1021/acsenergylett.6b00001

[37] Lissau J S, Viñas Muñoz A, Gotfredsen H, Jevric M, Nielsen M B and Sølling T I 2018 J. Phys. Chem. A 122 6683-6692 ISSN 1089-5639 URL https://doi.org/10.1021/acs. jpca.8b06064

[38] Zhou J, Liu Q, Feng W, Sun Y and Li F 2014 Chem. Rev. 115 395-465 ISSN 0009-2665 URL http://dx.doi.org/10.1021/cr400478f

[39] Englman R and Jortner J 1970 Mol. Phys. 18 145-164 ISSN 0026-8976 URL https://doi.org/10.1080/00268977000100171

[40] Singh-Rachford T N and Castellano F N 2008 J. Phys. Chem. A 112 3550-3556

[41] Rihter B D, Kenney M E, Ford W E and Rodgers M A J 1990 J. Am. Chem. Soc. 112 8064-8070 ISSN 0002-7863 URL https://doi.org/10.1021/ja00178a032

[42] Duan P, Yanai N, Nagatomi H and Kimizuka N 2015 J. Am. Chem. Soc. 137 1887-1894 ISSN 0002-7863 URL http://dx.doi.org/10.1021/ja511061h

[43] Hagstrom A L, Lee H L, Lee M S, Choe H S, Jung J, Park B G, Han W S, Ko J S, Kim J H and Kim J H 2018 ACS Appl. Mater. Interfaces 10 8985-8992 ISSN 1944-8244 URL https://doi.org/10.1021/acsami.7b17789

[44] Joarder B, Yanai N and Kimizuka N 2018 J. Phys. Chem. Lett. 9 4613-4624 ISSN 1948-7185 URL https://doi.org/10.1021/acs.jpclett.8b02172

[45] Li C, Koenigsmann C, Deng F, Hagstrom A, Schmuttenmaer C A and Kim J H 2016 ACS Photonics 3 784-790 URL https://doi.org/10.1021/acsphotonics.5b00694

[46] Wu M, Congreve D N, Wilson M W B, Jean J, Geva N, Welborn M, Van Voorhis T, Bulović V, Bawendi M G and Baldo M A 2015 Nat. Photonics 1031 URL https://doi.org/10.1038/nphoton.2015.226

[47] Monguzzi A, Mezyk J, Scotognella F, Tubino R and Meinardi F 2008 Phys. Rev. B 78195112

[48] Haefele A, Blumhoff J, Khnayzer R S and Castellano F N 2012 J. Phys. Chem. Lett. 299-303 ISSN 1948-7185 URL http://dx.doi.org/10.1021/jz300012u

[49] Jin J 2002 The Finite Element Method in Electromagnetics - Second Edition (John Wiley \& Sons Inc. New York)

[50] Larson M G and Bengzon F 2013 The Finite Element Method: Theory, Implementation, and Applications (Springer Publishing Company, Incorporated) ISBN 3642332862, 9783642332869

[51] Novotny L and Hecht B 2006 Principles of Nano-Optics (Cambridge University Press)

[52] Eriksen E H, Madsen S P, Julsgaard B, Hofmann C L M, Goldschmidt J C and Balling P 2019 Journal of Optics $\mathbf{2 1} 035004$

[53] Yu Z, Raman A and Fan S 2010 Proceedings of the National Academy of Sciences 107 17491-17496 ISSN 0027-8424 URL https://www.pnas.org/content/107/41/17491

[54] Fan X, Zheng W and Singh D J 2014 Light: Science ESAmp; Applications 3 e179 EP - review URL https://doi.org/10.1038/1sa.2014.60

[55] Jackson J D 1998 Classical Electrodynamics (Wiley) ISBN 9780471309321

[56] Pelton M and Bryant G W 2013 Introduction to metal-nanoparticle plasmonics A Wiley-Science Wise Co-Publication (Hoboken, NJ: Wiley) URL http://cds.cern.ch/record/1618678

[57] Yariv A and Yeh P 2007 Photonics : optical electronics in modern communications 
Improving the efficiency of upconversion by light concentration using nanoparticle design67

sixth edition ed (New York ; Oxford : Oxford University Press) ISBN 0195179463 revised edition of: Optical electronics in modern communications. 5th ed., (1997 URL http://hdl.library. upenn.edu/1017.12/366299

[58] Ogawa K, Chang W, Sopori B and Rosenbaum F 1973 IEEE Journal of Quantum Electronics 9 29-42 ISSN 0018-9197

[59] Johannsen S R, Madsen S P, Jeppesen B R, Nygaard J V, Julsgaard B, Balling P and Larsen A N 2015 Applied Physics Letters 106053101 URL https://doi.org/10.1063/1.4907415

[60] Bendsøe M P and Sigmund O 2003 Topology Optimization (Springer)

[61] Ginzburg P, Berkovitch N, Nevet A, Shor I and Orenstein M 2011 Nano Letters 11 2329-2333 pMID: 21528909 URL https://doi.org/10.1021/nl200612f

[62] Cox S J and Dobson D C 1999 SIAM J. Appl. Math. 59 2108-2120 ISSN 0036-1399 URL https://doi.org/10.1137/S0036139998338455

[63] Borel P I, Harpøth A, Frandsen L H, Kristensen M, Shi P, Jensen $\mathrm{J} \quad \mathrm{S}$ and Sigmund O 2004 Opt. Express 12 1996-2001 URL http://www.opticsexpress.org/abstract. cfm?URI=oe-12-9-1996

[64] Jensen J S and Sigmund O 2004 Applied Physics Letters 84 2022-2024 URL https://doi.org/10.1063/1.1688450

[65] Sigmund O and Hougaard K 2008 Phys. Rev. Lett. 100(15) 153904 URL https://link.aps.org/doi/10.1103/PhysRevLett.100.153904

[66] Andkjær J, Asger Mortensen N and Sigmund O 2012 Applied Physics Letters 100101106 URL https://doi.org/10.1063/1.3691835

[67] Andkjær J, Johansen V E, Friis K S and Sigmund O 2014 J. Opt. Soc. Am. B 31 164-174 URL http://josab.osa.org/abstract.cfm?URI=josab-31-1-164

[68] Dühring M B and Sigmund O 2013 J. Opt. Soc. Am. B 30 1154-1160 URL http://josab.osa.org/abstract. cfm?URI=josab-30-5-1154

[69] Wang F, Jensen J and Sigmund O 2012 Photonics and Nanostructures - Fundamentals and Applications 10 378-388 ISSN 1569-4410

[70] Vester-Petersen J, Madsen S P, Sigmund O, Balling P, Julsgaard $\mathrm{B}$ and Christiansen $\mathrm{R}$ E 2018 Opt. Express $\mathbf{2 6}$ A788-A795 URL http: //www . opticsexpress . org/abstract. cfm?URI=oe-26-18-A788

[71] Wang F, Christiansen R E, Yu Y, MÃ,rk J and Sigmund O 2018 Applied Physics Letters 113 241101 URL https://doi.org/10.1063/1.5064468

[72] Wadbro E and Engström C 2015 Computer Methods in Applied Me$\begin{array}{llllllll}\text { chanics and Engineering } & \mathbf{2 9 3} & 155 & - & 169 & \text { ISSN } & 0045-7825 & \text { URL }\end{array}$ http://www.sciencedirect.com/science/article/pii/S0045782515001589

[73] Pestourie R, Pérez-Arancibia C, Lin Z, Shin W, Capasso F and Johnson S G 2018 Opt. Express 26 33732-33747 URL http://www.opticsexpress.org/abstract.cfm?URI=oe-26-26-33732

[74] Lin Z, Liu V, Pestourie R and Johnson S G 2019 Opt. Express 27 15765-15775 URL http://www . opticsexpress . org/abstract. cfm?URI=oe-27-11-15765

[75] Christiansen R E, Wang F and Sigmund O 2019 Phys. Rev. Lett. 122(23) 234502 URL https://link.aps.org/doi/10.1103/PhysRevLett.122.234502

[76] Christiansen R E, Wang F, Sigmund O and Stobbe S 2019 To appear in Nanophotonics URL https://doi.org/10.1515/nanoph-2019-0057

[77] Jensen J and Sigmund O 2011 Laser 8 Photonics Reviews 5 308-321 URL https://onlinelibrary.wiley.com/doi/abs/10.1002/lpor.201000014

[78] Piggott A Y, Lu J, Lagoudakis K G, Petykiewicz J, Babinec T M and Vuckovic J 2015 Nature Photonics 9374 EP - URL https://doi.org/10.1038/nphoton.2015.69

[79] Shen B, Wang P, Polson R and Menon R 2015 Nature Photonics 9378 EP - URL https://doi.org/10.1038/nphoton.2015.80

[80] Sigmund O, Jensen J S and Frandsen L H 2016 Nature Photonics 10142 EP - correspondence URL https://doi.org/10.1038/nphoton.2016.26 
Improving the efficiency of upconversion by light concentration using nanoparticle design68

[81] Molesky S, Lin Z, Piggott A Y, Jin W, Vuckovic J and Rodriguez A W 2018 Nature Photonics 12 659-670 ISSN 1749-4893 URL https://doi.org/10.1038/s41566-018-0246-9

[82] Lalau-Keraly C M, Bhargava S, Miller O D and Yablonovitch E 2013 Opt. Express 21 21693-21701 URL http://www.opticsexpress.org/abstract.cfm?URI=oe-21-18-21693

[83] Niederberger A C R, Fattal D A, Gauger N R, Fan S and Beausoleil R G 2014 Opt. Express 22 12971-12981 URL http://www . opticsexpress .org/abstract.cfm?URI=oe-22-11-12971

[84] Sigmund O 2011 Structural and Multidisciplinary Optimization 43 589-596 ISSN 1615-1488 URL https://doi.org/10.1007/s00158-011-0638-7

[85] Shim H, Fan L, Johnson S G and Miller O D 2019 Phys. Rev. X 9(1) 011043 URL https://link.aps.org/doi/10.1103/PhysRevX.9.011043

[86] Kan Y, Rohit U and Yuebing Z 2019 Intelligent nanophotonics: merging photonics and artificial intelligence at the nanoscale vol 8 (De Gruyter, Berlin/Boston) chap Nanophotonics, p $339 \quad 3$ URL https://www. degruyter.com/view/j/nanoph.2019.8. issue-3/nanoph-2018-0183/nanoph-2018-0183.xml

[87] Nocedal J and JWright S 2006 Numerical Optimization - Second Edition (Springer Science+Business Media LCC)

[88] Svanberg K 2002 Siam Journal on Optimization 12 555-573

[89] Aage N, Andreassen E, Lazarov B S and Sigmund O 2017 Nature 550 84-86

[90] Tortorelli D A and Michaleris P 1994 Inverse Problems in Engineering 1 71-105

[91] Bourdin B 2001 Int. J. Numer. Meth. Eng. 50 2143-2158

[92] Bruns T E and Tortorelli D A 2001 Computer Methods in Applied Mechanics and Engineering $1903443-3459$

[93] Guest J K, Prévost J H and Belytschko T 2004 International Journal for Numerical Methods in Engineering $61238-254$

[94] Xu S, Cai Y and Cheng G 2010 Structural Multidiciplinary Optimization 41 495-505

[95] Wang F, Lazarov B S and Sigmund O 2011 Struct. Multidiscip. O. 43 767-784

[96] Christiansen R E, Lazarov B S, Jensen J S and Sigmund O 2015 Structural and Multidisciplinary Optimization $\mathbf{5 2}$ 737-754

[97] Lazarov B S and Sigmund O 2010 Numerical Methods in Engineering 86(6) 765-781

[98] Vester-Petersen J, Christiansen R E, Julsgaard B, Balling P, Sigmund O and Madsen S P 2017 Applied Physics Letters 111133102

[99] Christiansen R E, Vester-Petersen J, Madsen S P and Sigmund O 2019 Computational Methods for Applied Mechanical Engineering 343 23-39

[100] Wind S J, Gerber P D and Rothuizen H 1998 J. Vac. Sci. Technol. B 16 3262-3268

[101] Parikh M 1978 J. Vac. Sci. Technol. B 15 931-933

[102] Cui Z 2017 Nanofabrication by Electron Beam (Cham: Springer International Publishing) pp 91-148 ISBN 978-3-319-39361-2

[103] Pavkovich J M 1986 J. Vac. Sci. Technol. B 4 159-163

[104] Murai F, Yoda H, Okazaki S, Saitou N and Sakitani Y 1992 J. Vac. Sci. Technol. B 10 3072-3076

[105] Haslam M E and McDonald J F 1986 J. Vac. Sci. Technol. B 4 168-175

[106] Peckerar M C, Chang S and Marrian C R K 1995 J. Vac. Sci. Technol. B 13 2518-2525

[107] Poonawala A and Milanfar P 2007 IEEE Trans. Image Process. 16 774-788 ISSN 1057-7149

[108] Zhou M, Lazarov B S and Sigmund O 2014 Appl. Opt. 53 2720-2729

[109] Sewell H 1978 J. Vac. Sci. Technol. 15 927-930

[110] Kratschmer E 1981 J. Vac. Sci. Technol. 19 1264-1268

[111] Owen G and Rissman P 1983 J. Appl. Phys. 54 3573-3581

[112] Groves T R 1993 J. Vac. Sci. Technol. B 11 2746-2753

[113] Osawa M, Takahashi K, Sato M, Arimoto H, Ogino K, Hoshino H and Machida Y 2001 J. Vac. Sci. Technol. B 19 2483-2487

[114] Klimpel T, Schulz M, Zimmermann R, Stock H J and Zepka A 2011 J. Vac. Sci. Technol. B 29 $06 \mathrm{~F} 315$ 
Improving the efficiency of upconversion by light concentration using nanoparticle design69

[115] Ocola L E, Gosztola D J, Rosenmann D and Lopez G 2015 J. Vac. Sci. Technol. B 33 06FD02

[116] Nien C, Chang L C, Ye J H, Su V C, Wu C H and Kuan C H 2017 J. Vac. Sci. Technol. B 35 051603

[117] Eriksen E H, Nazir A, Balling P, Vester-Petersen J, Christiansen R E, Sigmund O and Madsen S P 2018 Microelectron. Eng. $19952-57$

[118] pecpy 0.6 python package https://pypi.org/project/pecpy/ accessed: 2019-04-09

[119] Lee S Y and Anbumony K 2006 Microelectron. Eng. 83 336-344

[120] Schift H 2008 J. Vac. Sci. Technol. B 26458 URL https://doi.org/10.1116/1.2890972

[121] Kooy N, Mohamed K, Pin L and Guan O 2014 Nanoscale Res. Lett. 9320 URL https://doi.org/10.1186/1556-276x-9-320

[122] Sreenivasan S 2017 Microsyst. Nanoeng. 3 URL https://doi .org/10.1038/micronano.2017.75

[123] Kreibig U and Vollmer M 1995 Optical Properties of Metal Clusters (Springer Berlin Heidelberg) ISBN 978-3-642-08191-0 URL https://doi.org/10.1007/978-3-662-09109-8

[124] Bohren C F and Huffman D R 1983 Absorption and scattering of light by small particles (John Wiley \& Sons)

[125] Møller S H, Vester-Petersen J, Nazir A, Eriksen E H, Julsgaard B, Madsen S P and Balling P 2018 Appl. Phys. A 124 URL https://doi.org/10.1007/s00339-018-1615-4

[126] Leiderer P, Bartels C, König-Birk J, Mosbacher M and Boneberg J 2004 Appl. Phys. Lett. 85 5370-5372 URL https://doi.org/10.1063/1.1819990

[127] Fiutowski J 2012 J. Nanophotonics 6063515 URL https://doi.org/10.1117/1.jnp.6.063515

[128] Dickreuter S, Gleixner J, Kolloch A, Boneberg J, Scheer E and Leiderer P 2013 Beilstein J. Nanotechnol. 4 588-602 URL https://doi.org/10.3762/bjnano.4.66

[129] Kühler P, de Abajo F J G, Leiprecht P, Kolloch A, Solis J, Leiderer P and Siegel J 2012 Opt. Express 2022063 URL https://doi.org/10.1364/oe.20.022063

[130] David C, Kühler P, de Abajo F J G and Siegel J 2014 Opt. Express 228226 URL https://doi.org/10.1364/oe.22.008226

[131] Deeb C, Bachelot R, Plain J, Baudrion A L, Jradi S, Bouhelier A, Soppera O, Jain P K, Huang L, Ecoffet C, Balan L and Royer P 2010 ACS Nano 4 4579-4586 URL https://doi.org/10.1021/nn101017b

[132] Geldhauser T, Kolloch A, Murazawa N, Ueno K, Boneberg J, Leiderer P, Scheer E and Misawa H 2012 Langmuir 28 9041-9046 URL https://doi.org/10.1021/la300219w

[133] Youn S W, Ueno A, Takahashi M and Maeda R 2008 Microelectron. Eng. 85 1924-1931 URL https://doi.org/10.1016/j.mee.2008.06.016

[134] Yu R, Liz-Marzán L M and de Abajo F J G 2017 Chem. Soc. Rev. 46 6710-6724 URL https://doi.org/10.1039/c6cs00919k

[135] Schulze T F, Czolk J, Cheng Y Y, Fückel B, MacQueen R W, Khoury T, Crossley M J, Stannowski B, Lips K, Lemmer U, Colsmann A and Schmidt T W 2012 J. Phys. Chem. C 116 22794-22801 ISSN 1932-7447 URL https://doi.org/10.1021/jp309636m

[136] Adikaari A A D, Etchart I, Guéring P H, Bérard M, Silva S R P, Cheetham A K and Curry R J 2012 Journal of Applied Physics 111094502 ISSN 0021-8979 URL https://doi.org/10.1063/1.4704687

[137] Chen W, Hou Y, Osvet A, Guo F, Kubis P, Batentschuk M, Winter B, Spiecker E, Forberich K and Brabec C J 2015 Organic Electronics 19 113-119 ISSN 1566-1199 URL http://www.sciencedirect.com/science/article/pii/S1566119915000476

[138] Wang H Q, Stubhan T, Osvet A, Litzov I and Brabec C J 2012 Solar Energy Materials and Solar Cells 105 196-201 ISSN 0927-0248 URL http://www.sciencedirect.com/science/article/pii/S0927024812002838

[139] Ahmadpour M, Fernandes Cauduro A L, Méthivier C, Kunert B, Labanti C, Resel R, Turkovic V, Rubahn H G, Witkowski N, Schmid A K and Madsen M 2019 ACS Appl. Energy Mater. 2 420-427 URL https://doi.org/10.1021/acsaem.8b01452

[140] Mirsafaei M 2019 Sputter deposited titanium oxide layers as efficient electron selective contacts 
Improving the efficiency of upconversion by light concentration using nanoparticle design70

in organic photovoltaic devices under review

[141] Mirsafaei M, Fallahpour A H, Lugli P, Rubahn H G, Adam J and Madsen M 2017 Scientific Reports 75300 ISSN 2045-2322 URL https://doi.org/10.1038/s41598-017-05591-8 